\title{
Measurement of the Spin Correlation in the Top Quark Pair Production using the Dilepton Events in 1.96-TeV Proton-Antiproton Collisions
}

Kenichi TAKEMASA

February 2012 



\title{
Measurement of the Spin Correlation in the Top Quark Pair Production using the Dilepton Events in 1.96-TeV Proton-Antiproton Collisions
}

\author{
Kenichi TAKEMASA \\ (Doctoral Program in Physics)
}

Submitted to the Graduate School of

Pure and Applied Sciences

in Partial Fulfillment of the Requirements for the Degree of Doctor of Philosophy in

Science

at the

University of Tsukuba 



\begin{abstract}
One of the most remarkable properties of the top quark is its extremely short lifetime, which allows us to observe the top quark spin at its production. That means a spin correlation at $t \bar{t}$ production is possible to be observed. In this thesis, we report on a measurement of the correlation coefficient between top quark spin and antitop quark spin in the beam basis at top quark pair production. We select $t \bar{t}$ pair production candidates by requiring two high transverse momentum leptons, two jets and large missing $E_{T}$. In order to reconstruct the angular distribution of top quark decay products, we perform full kinematical reconstruction using predicted distributions of $p_{z}^{t \bar{t}}, p_{T}^{t \bar{t}}$ and $M_{t \bar{t}}$. We make signal and background templates from admixture of Monte Carlo simulations and data-based background modelings. Then, we perform unbinned likelihood fit of angular distribution of data to signal and background templates and obtain result. This analysis is based on the data of $5.1 \mathrm{fb}^{-1}$ collected with the Collider Detector Facility (CDF) at the Fermilab Tevatron between March 2002 and June 2009. We observe $334 t \bar{t}$ candidate events. We determine a confidence interval at $68 \%$ level for the correlation coefficient $\kappa$ to be $-0.520<\kappa<0.605$ or $\kappa=0.042_{-0.562}^{+0.563}$ on the assumption of $M_{\text {top }}=172.5 \mathrm{GeV} / c^{2}$.
\end{abstract}




\section{Acknowledgments}

I would like to thank people who have helped me and contribute to my growth in my college life.

First of all, I would like to acknowledge a great debt to my advisor, Prof. Shinhong Kim and Prof. Yuji Takeuchi for giving me the opportunity to work on CDF and suggesting the topic of this analysis. They have gave me a lot of adivices and always guided me in the right direction. Without their support and encouragement, this thesis would not have been possible.

I wish to thank staff of the High Energy Physics group of University of Tsukuba, Prof. Fumihiko Ukegawa, Prof. Kazuhiko Hara, Prof. Koji Sato, Dr. Hideki Miyake, Dr. Masakazu Kurata, Dr. Koichi Nagai for their kindness. They give me a lot of suggestions to my analysis. I also thank students who belong to the High Energy Physics group of University of Tsukuba. I shared good times with them. In particular, I would like to thank Yuji Sudo who is my peer. He listened to my problem on analysis and supported my life at Fermilab. Also, I would like to thank academic colleague at Fermilab, Atsunari Hamaguchi, Daisuke Yamato, Yuki Sakurai, Yujiro Funakoshi, and people who belonged the High Energy Physics group of University of Tsukuba, Prof. Takasumi Maruyama, Dr. Naoki Kimura, Dr. Tomonobu Tomura, Dr. Junji Naganoma, Masato Aoki and Dr. Yoshikazu Nagai. They gave me a lot of advices and provided motivation to analysis. I wish to express apprecisation to Kazuko Kumashiro, Kyoko Kunori for their constant support with their secretarial works.

Conveners of CDF Top Group and Dilepton working group gave me a lot of helps. My deep thank go to the members of CDF top dilepton working group, Jaroslav Antos, Yen Chu Chen, Hyunsoo Kim, Chang-Seong Moon, Youngdo Oh, Andy Beretvas, Soo-Bong Kim, Roman Lysak and Satoru Uozumi. I thank the Fermilab staff and the technical staffs of the participating insttutions for their vital contributions.

I wish to thank my best friend Junichi Tanaka. Playing with him was a nice change of pace for me.

Finally, I would like to thank my parents and my sister. 


\section{Contents}

1 Introduction 1

1.1 The Standard Model . . . . . . . . . . . . . . . . . . . . . 2

1.2 The Spin Quantum Number . . . . . . . . . . . . . . . . . . . . 5

1.3 The Top Quark . . . . . . . . . . . . . . . . . . . . . 5 5

1.4 Top Quark Production . . . . . . . . . . . . . . . . . . . . . . 6

1.5 Top Quark Decay . . . . . . . . . . . . . . . . . . . . . . . . 7

1.6 Top Quark Mass . . . . . . . . . . . . . . . . . . . . . . . . . . . . 9

1.7 Top Quark Spin Correlation . . . . . . . . . . . . . . . . . . . . . 10

$1.8 \kappa^{\text {meas }}$, what is measured in this analysis . . . . . . . . . 12

1.9 Motivation of Spin Correlation Measurement . . . . . . . . . . . 13

1.10 Previous Measurement of Top Quark Spin Correlation . . . . . . . . . . . 13

1.10 .1 Dilepton channel . . . . . . . . . . . . . . . . . . 13

1.10 .2 Lepton+Jets channel . . . . . . . . . . . . . . . . . . 14

1.10.3 Combine lepton+Jets events and dilepton events . . . . . . . . 14

1.11 Outline of This Thesis . . . . . . . . . . . . . . . . . 14

2 Experimental Apparatus $\quad 15$

2.1 The Fermilab Accelerator Complex _. . . . . . . . . . . . . 15

2.2 The CDF II Detector . . . . . . . . . . . . . . . . . . . . . . . . 19

2.2 .1 Tracking System $\ldots \ldots \ldots \ldots \ldots$

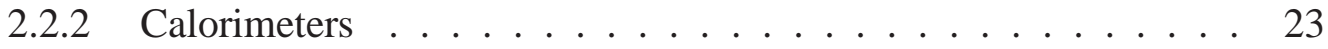

2.2 .3 Muon Detectors . . . . . . . . . . . . . . . . . . . . . 26

2.2 .4 Luminosity Monitor . . . . . . . . . . . . . . . . . 27

2.2.5 Data Acquisition System . . . . . . . . . . . . . . . 28

3 Reconstruction and Event Selection 31

3.1 Electron Identification $\ldots \ldots \ldots \ldots . \ldots \ldots$

3.1 .1 Central Electrons . . . . . . . . . . . . . . . . . . . 31

3.1 .2 Plug Electrons $\ldots \ldots \ldots \ldots \ldots$

3.2 Muon Identification . . . . . . . . . . . . . . . . . . . . . . 37

3.3 Jet Reconstruction . . . . . . . . . . . . . . . . . . . . . . . . . . . . . . 39

3.3.1 Jet Energy Corrections . . . . . . . . . . . . . . . . . 40

3.3.2 Jet Energy Scale Uncertainties . . . . . . . . . . . . . . . . 42

3.4 Missing Energy . . . . . . . . . . . . . . . . . . . 42 
4 Spin Correlation Measurement $\quad \mathbf{4 5}$

4.1 Outline of the Measurement . . . . . . . . . . . . . . . . 45

4.2 Top Dilepton Event Selection . . . . . . . . . . . . . . . . . . 45

4.3 Background Composition . . . . . . . . . . . . . . . . . 47

4.4 Full Kinematical Reconstruction in Dilepton Channel . . . . . . . . . . . 49

4.4.1 Principle ........................ 49

4.4 Likelihood . . . . . . . . . . . . . . . . 49

4.4.3 Jet and MET Resolutions . . . . . . . . . . . . . . . 50

4.4.4 Performance of the Full Kinematical Reconstruction Method . . . 51

4.5 Templates of the Signal and the Background . . . . . . . . . . . . . 53

4.5.1 Template Fit Function . . . . . . . . . . . . . . . 53

4.5.2 Signal Template as a Function of $\kappa \ldots . . \ldots 53$

4.5.3 Background Template . . . . . . . . . . . . . 57

4.5.4 Crosscheck of the Signal and the Background shape . . . . . . . 64

4.6 Pseudo-experiments . . . . . . . . . . . . . . . . 65

4.6.1 Check of the Method for $\kappa$ Measurement . . . . . . . . . . 67

4.6.2 Pseudo Experiment Results . . . . . . . . . . . . . . . 69

4.6.3 Correlation between $\kappa^{\text {meas }}$ from leptons and $\kappa^{\text {meas }}$ from $b$-jets $\quad . \quad 71$

4.6.4 Construction of Feldman-Cousins intervals . . . . . . . . . 73

5 Systematic Uncertainties $\quad 75$

5.1 Statistical Uncertainty of Signal MC Sample . . . . . . . . . . . . . 75

5.2 Uncertainties in expected signal, background numbers and background templates . . . . . . . . . . . . . . . . . 75

5.3 Jet Energy Scale Uncertainty . . . . . . . . . . . . . . . . . . . . . . . . . . . . . . . 77

5.4 Initial and Final State Radiation . . . . . . . . . . . . . . . . . 79

5.5 Uncertainty of $g \boldsymbol{g}$ fraction . . . . . . . . . . . . . 80

5.6 Parton Distribution Functions . . . . . . . . . . . . . . . . 80

5.7 Systematics from $\mathrm{LO}$ versus $\mathrm{NLO} \ldots \ldots . \ldots . \ldots . . \ldots 81$

5.8 Color Reconnection . . . . . . . . . . . . . . . . . . . . . . . . 82

5.9 Total Uncertainty and Feldman-Cousins Intervals . . . . . . . . . . . 84

$\begin{array}{lll}6 & \text { Results and Discussions } & 87\end{array}$

$\begin{array}{llr}7 & \text { Conclusion } & 91\end{array}$

A Spin States at $t \bar{t}$ Pair Production $\quad 93$

A.1 Production by $q \bar{q} \rightarrow t \bar{t} \ldots \ldots \ldots . \ldots \ldots 3$

A.2 Production by $\boldsymbol{g} \boldsymbol{g} \rightarrow \boldsymbol{t} \overline{\boldsymbol{t}} \ldots \ldots \ldots . \ldots \ldots 5$

B P, CP of $t \bar{t}$ System 97 


\section{List of Figures}

$1.1 \quad$ Leading-order production diagram for $q \bar{q} \rightarrow t \bar{t} . \ldots \ldots \ldots$

1.2 Leading-order production diagrams for $g g \rightarrow t \bar{t} \ldots \ldots \ldots \ldots$

1.3 The spin configurations of the gluon fusion and the $q \bar{q}$ annihilation sub-

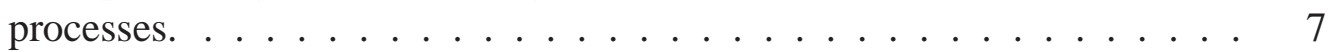

1.4 NLO calculations of $\sigma(p \bar{p} \rightarrow t \bar{t} X) \ldots \ldots \ldots \ldots \ldots \ldots$

1.5 Tree level $q \bar{q} \rightarrow t \bar{t}$ production and decay. . . . . . . . . . . 8

1.6 Loop diagrams generating corrections to the theoretical $W$ boson mass. . 9

1.7 Electroweak constraints on the Higgs boson mass. . . . . . . . . . . 11

1.8 The two-dimensional distributions of $\cos \theta_{+}$and $\cos \theta_{-}$in beamline basis for the processes $q \bar{q} \rightarrow t \bar{t}$ and $g g \rightarrow t \bar{t}$. . . . . . . . . . . . 12

2.1 A diagram of the Fermilab accelerator chain. . . . . . . . . . . 16

2.2 Isometric view of the CDF II detector. . . . . . . . . . . . . 20

2.3 The $\mathrm{CDF}$ tracking system. . . . . . . . . . . . . . . . . 21

2.4 The CDF silicon detector . . . . . . . . . . . . . . . . . 22

2.5 The $\mathrm{CDF}$ calorimeter system. . . . . . . . . . . . . . . . . . . . . 24

2.6 The single CEM wedge. . . . . . . . . . . . . . . . . 25

2.7 CDF muon coverage for the CMU, CMP, CMX, and BMU detectors. The BMU is referred as the IMU. . . . . . . . . . . . . . . . . . . 26

2.8 A cross section view of a CMU muon chamber. . . . . . . . . . . 26

2.9 Total integrated luminosity delivered by the Tevatron and recorded by the CDF. . . . . . . . . . . . . . . . . . . . . 28

2.10 Data flow in the CDF data acquisition system. . . . . . . . . . . . 29

2.11 Block diagram of the Level 1 and Level 2 trigger path. . . . . . . . . . 30

3.1 The total uncertainties of JES. . . . . . . . . . . . . . . . . 43

4.1 Distributions of $p_{z}^{t \bar{t}}, p_{T}^{t \bar{t}}$, and $M_{t \bar{t}}$ of dilepton candidates in $t \bar{t}$ PYTHIA Monte Carlo . . . . . . . . . . . . . . . . . . . . . 50

4.2 Mean and $\sigma$ of the distribution of $\left(E_{T}^{\text {true }}-E_{T}^{\mathrm{L} 5}\right) / E_{T}^{\mathrm{L} 5}$ as a function of $E_{T}^{\mathrm{L} 5}$

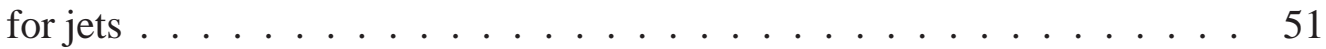

$4.3 \sigma$ of the distribution of $E_{x, y}^{\text {meas }}-E_{x, y}^{\text {true }}$ as a function of $E_{T}^{\text {jet1 }}+E_{T}^{\text {jet2 }} \ldots \quad . \quad 52$

4.4 Scatter plots of reconstructed kinematical variables versus the true value of the corresponding variables. . . . . . . . . . . . . . 52

4.5 The distribution of reconstructed $\left(\cos \theta_{+}, \cos \theta_{-}\right)$and $\left(\cos \theta_{b}, \cos \theta_{\bar{b}}\right)$ on the assumption of $\kappa=+1 \ldots \ldots \ldots \ldots \ldots 4$ 
4.6 The distributions of reconstructed $\left(\cos \theta_{+}, \cos \theta_{-}\right)$and $\left(\cos \theta_{b}, \cos \theta_{\bar{b}}\right)$ on the assumption of $\kappa=0 . \ldots \ldots \ldots 55$

4.7 The distributions of reconstructed $\left(\cos \theta_{+}, \cos \theta_{-}\right)$and $\left(\cos \theta_{b}, \cos \theta_{\bar{b}}\right)$ on the assumption of $\kappa=-1 \ldots \ldots \ldots \ldots 5$

$4.8 \kappa$ dependence of the fit parameters $\left(C_{i}^{\ell}(\kappa)\right)$ for the $\left(\cos \theta_{+}, \cos \theta_{-}\right)$distri-

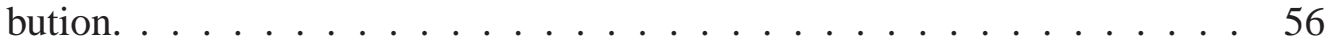

$4.9 \kappa$ dependence of the fit parameters $\left(C_{i}^{b}(\kappa)\right)$ for the $\left(\cos \theta_{b}, \cos \theta_{\bar{b}}\right)$ distri-

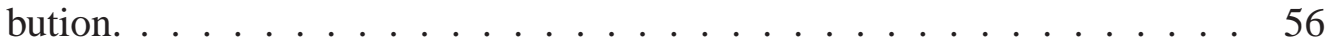

4.10 The distributions of $W W / W Z / Z Z$ background . . . . . . . . . . 57

4.11 The distributions of $Z \rightarrow \tau \tau$ background . . . . . . . . . . . . . 59

4.12 The distributions of $Z / \gamma^{*} \rightarrow e e, \mu \mu$ background . . . . . . . . . . . 62

4.13 The distribution of fake background . . . . . . . . . . . . . 62

4.14 The distribution of all backgrounds . . . . . . . . . . . . . 63

4.15 The distribution of $\cos \theta_{+}, \cos \theta_{-}$and $\cos \theta_{b}, \cos \theta_{\bar{b}} \ldots \ldots \ldots 4$

4.16 An example of pseudo data . . . . . . . . . . . . . . 66

4.17 The distribution of reconstructed $\left(\cos \theta_{+}, \cos \theta_{-}\right)$and $\left(\cos \theta_{b}, \cos \theta_{\bar{b}}\right)$ in Herwig Monte Carlo . . . . . . . . . . . . . . . . . . 67

4.18 The distribution of $\kappa^{\text {meas }}$ of pseudo-experiments using herwig Monte Carlo as signal sample pool, on assumption of $\mathrm{N}_{\mathrm{bkg}}=0 \ldots \ldots$. . . . . . . . . 67

4.19 The distribution of $\kappa^{\text {meas }}$ of pseudo-experiments using herwig Monte Carlo as signal sample pool . . . . . . . . . . . . . . . . . . . 68

4.20 The result of pseudo-experiments . . . . . . . . . . . . . . . . 69

4.21 The pull distribution of pseudo-experiments . . . . . . . . . . . . 70

$4.22 \sigma\left(\kappa^{\text {meas }}\right)$ as a function of $\kappa^{\text {true }}$ for leptons only case. . . . . . . . . . . . 71

$4.23 \sigma\left(\kappa^{\text {meas }}\right)$ as a function of $\kappa^{\text {true }}$ for b-jets only case. . . . . . . . . . 71

4.24 Feldman-Cousins confidence interval (statistics only) . . . . . . . . 73

5.1 Statistical uncertainty of signal Monte Carlo sample . . . . . . . . . . 76

5.2 Uncertainties in expected signal, background numbers and background templates . . . . . . . . . . . . . . . . 78

5.3 Jet energy scale uncertainty . . . . . . . . . . . . . . . . . . 78

5.4 Diagram of $t \bar{t}$ production and decay with ISR and FSR . . . . . . . . . 79

5.5 ISR/FSR uncertainty . . . . . . . . . . . . . . . . . . . . 79

5.6 Uncertainty of $g g$ fraction . . . . . . . . . . . . . . . . . . 80

5.7 PDF uncertainty . . . . . . . . . . . . . . . . . . . . 82

5.8 Feynman diagrams of $t \bar{t}$ production for $\mathrm{LO}$ and $\mathrm{NLO} \ldots \ldots 3$

5.9 Systematics from LO versus NLO . . . . . . . . . . . . . . . 83

5.10 Systematics from color reconnection . . . . . . . . . . . . . 83

5.11 Total systematic uncertainty . . . . . . . . . . . . . . . . . . . 84

5.12 Total uncertainty . . . . . . . . . . . . . . . . . . . 85

5.13 Feldman-Cousins confidence interval (statistics+systematics) . . . . . 85

6.1 The distribution of $\left(\cos \theta_{+}, \cos \theta_{-}\right)$and $\left(\cos \theta_{b}, \cos \theta_{\bar{b}}\right)$ in data for integrated luminosity of $5.1 \mathrm{fb}^{-1} \ldots \ldots \ldots$. . . . . . . . . . . 87

6.2 Feldman-Cousins confidence intervals with measured result . . . . . . . 88 
6.3 Mass dependence on $\kappa$ measurement . . . . . . . . . . . . . . . . . 89

A.1 Differential cross section for $t \bar{t}$ production as a function of the $t \bar{t}$ invariant mass for the Tevatron . . . . . . . . . . . . . . . . 96

B.1 Spin configurations of $t \bar{t}$ system and their $\mathrm{P}$ and CP reversal states . . . 97 



\section{List of Tables}

1.1 Fundamental properties of fermions. . . . . . . . . . . . 3

1.2 Fundamental properties of bosons. . . . . . . . . . . . . . . . 4

4.1 The number of expected events in data correspond to $5.1 \mathrm{fb}^{-1}$ with the observed number of events . . . . . . . . . . . . . . . . . . . . . . . . . . 48

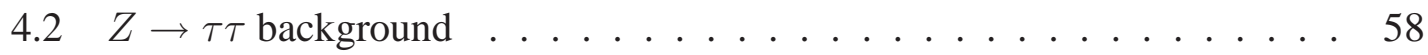

4.3 ee channels of Drell-Yan background . . . . . . . . . . . . . 60

$4.4 \mu \mu$ channels of Drell-Yan background. . . . . . . . . . . . 61

5.1 The summary table of systematic uncertainty . . . . . . . . . . . 84 



\section{Chapter 1}

\section{Introduction}

Particle physics is a branch of physics. Its purpose is to understand the fundamental constituents of matters and the laws governing them. This is done by studying the interactions between objects, which can range from something as large as whole galaxies to the smallest building blocks of matter such as quark. The fundamental interaction between quarks and other elementary particles that form everything we see today is described by particle physics. As of now, the Standard Model (SM), a theory that describes these elementary particle interactions, has been very successful due to results of high energy experiments which have thoroughly verified the accuracy of the Standard Model.

Particle physics have been using particle colliders to investigate the fundamental particles and the interactions. The Tevatron is a proton-antiproton collider located at the Fermi National Accelerator Laboratory (Fermilab). It is a powerful enough to produce top quarks. At the Tevatron, top quarks are predicted to be produced in pairs via the strong force, and decay via the electroweak force. The top quark is by far the heaviest fundamental particle in the Standard Model. Because of its large mass, the top quark has several unique properties and could provide hints for the original of mass and physics beyond the Standard Model.

Top quark and tau neutrino are observed in 1995 [1,2] and in 2000 [3], respectively. By these two observations, almost all of the Standard Model particles has been observed directly, except only the Higgs boson. Despite the great success of the Standard Model in describing the particles and interactions observed up to now, however there is a good theoretical motivation to believe that a new framework must come into play at approximately the $\mathrm{TeV}$ energy scale. Increasingly stringent measurements of the properties of particles and their interactions at the highest available energies, coupled with direct searches for phenomena not described by the Standard Model, are the main project of modern experimental particle physics. A significant part of the project is the elucidation of the properties of the top quark.

This thesis reports measurements of the correlation coefficient between top quark spin and anti-top quark spin. A measurement of the spin correlation coefficient plays an important role in testing the Standard Model. 


\subsection{The Standard Model}

The Standard Model of elementary particle physics is a quantum field theory that describes the fundamental particles and the interactions between them. It includes the strong, weak and electromagnetic force. The model itself is a combination of the theory of quantum chromodynamics (QCD) $[4,5]$ and the Glashow-Salam-Weinberg (GSW) theory of electroweak interactions [6-8].

The strong force is described by quantum chromodynamics (QCD), a quantum field theory with an $S U(3)_{C}$ gauge group, and an asymptotically free coupling. Asymptotic freedom means that the strong force is weak at high energies or small distances, and only become strong at low energies or large distances. The strong force becomes strong enough at large distances that when colored quarks and gluons become separated, the binding energy between them is large enough to create new quarks and gluons. All the free quarks and gluons ultimately become bound into hadrons. This process of hadronization has a characteristic scale of $\Lambda_{Q C D} \sim 200 \mathrm{MeV}$, which corresponds to a time scale of $\tau_{t} \sim 10^{-24} \mathrm{~s}$.

The electromagnetic and weak forces are combined together into a single electroweak force which obeys an $S U(2)_{L} \times U(1)_{Y}$ gauge group. The $S U(2)_{L}$ group has three generators and the $U(1)_{Y}$ group has one, corresponding to four gauge bosons that mediate the electroweak force: $W_{\mu}^{1}, W_{\mu}^{2}, W_{\mu}^{3}$ for $S U(2)_{L}$ and $B_{\mu}$ for $U(1)_{Y}$. The $S U(2)_{L} \times$ $U(1)_{Y}$ symmetry of the electroweak interaction is spontaneously broken through its coupling to the scalar higgs field. The higgs field has a degenerate ground state at a finite value of the field and thus spontaneously breaking turn into three massive gauge bosons $\left(W^{+}, W^{-}, Z^{0}\right)$ and the massless photon which obeys an unbroken $U(1)_{E M}$ gauge symmetry.

The Standard Model contains the strong interaction which is represented as $S U(3)_{C}$ and the electroweak interaction which is represented as $S U(2)_{L} \otimes U(1)_{Y}$. Therefore, the Standard Model is described as follows, and is locally invariant under transformations of the group.

$$
G=S U(3)_{C} \otimes S U(2)_{L} \otimes U(1)_{Y}
$$

There is another known force in the nature, graviton, but its interaction is too weak to be detected in the subatomic experiments. Therefore gravitation is not understood in terms of particle physics, and has not been included the Standard Model.

The fundamental particles are categorized into two categories, spin $s=\frac{1}{2}$ fermions which are the constituents of normal matter, and spin $s=1$ bosons which mediate the force between fermions.

Spin $s=\frac{1}{2}$ fermions are classified into quarks and leptons. There are six types of quarks: up $(u)$, down $(d)$, charm $(c)$, strange $(s)$, top $(t)$ and bottom $(b)$. Similarly, there are six types of leptons: electron $(e)$, muon $(\mu)$, tau $(\tau)$, and their respective neutrinos $\left(\nu_{e}, \nu_{\mu}, \nu_{\tau}\right)$. These fermions are classified into the three generations of left-handed and right-handed quarks and leptons. The left-handed fermions are in weak isospin doublets, while the right-handed fermions are in weak isospin singlets:

1st generation: $L_{e}=\left(\begin{array}{c}\nu_{e} \\ e^{-}\end{array}\right)_{L}, R_{e}=e_{R}^{-}, L_{q}^{(1)}=\left(\begin{array}{c}u \\ d^{\prime}\end{array}\right)_{L}, R_{u}^{(1)}=u_{R}, R_{d}^{(1)}=d_{R}$ 
2nd generation: $L_{\mu}=\left(\begin{array}{c}\nu_{\mu} \\ \mu^{-}\end{array}\right)_{L}, R_{\mu}=\mu_{R}^{-}, L_{q}^{(2)}=\left(\begin{array}{c}c \\ s^{\prime}\end{array}\right)_{L}, R_{u}^{(2)}=c_{R}, R_{d}^{(2)}=s_{R}$

3rd generation: $L_{\tau}=\left(\begin{array}{c}\nu_{\tau} \\ \tau^{-}\end{array}\right)_{L}, R_{\tau}=\tau_{R}^{-}, L_{q}^{(3)}=\left(\begin{array}{c}t \\ b^{\prime}\end{array}\right)_{L}, R_{u}^{(3)}=t_{R}, R_{d}^{(3)}=b_{R}$

These fermions are characterized with weak isospin $(I)$ and weak hypercharge $(Y)$ through the relation $Q=I_{3}+\frac{1}{2} Y$, where $Q$ is the electric charge. The mass eigenstates of the left-handed down-type quarks $\left(d^{\prime}, s^{\prime}, b^{\prime}\right)$ are related to flavor eigenstates $(d, s, b)$ through the Cabibbo-Kobayashi-Masukawa matrix [9, 10]:

$$
\left(\begin{array}{l}
d^{\prime} \\
s^{\prime} \\
b^{\prime}
\end{array}\right)=\left(\begin{array}{lll}
V_{u d} & V_{u s} & V_{u b} \\
V_{c d} & V_{c s} & V_{c b} \\
V_{t d} & V_{t s} & V_{t b}
\end{array}\right)\left(\begin{array}{c}
d \\
s \\
b
\end{array}\right)
$$

The fundamental properties of fermions are summarized in Table 1.1.

\begin{tabular}{|c|c|c|c|c|}
\hline & Particle & Electric charge $Q$ & Hypercharge $Y$ & Mass \\
\hline \multirow{6}{*}{ Quarks } & $\begin{array}{l}u_{L} \\
u_{R}\end{array}$ & $+2 / 3$ & $\begin{array}{l}1 / 3 \\
4 / 3\end{array}$ & $1.7-3.1 \mathrm{MeV} / c^{2}$ \\
\hline & $\begin{array}{l}d_{L} \\
d_{R}\end{array}$ & $-1 / 3$ & $\begin{array}{c}1 / 3 \\
-2 / 3\end{array}$ & $3.0-4.8 \mathrm{MeV} / c^{2}$ \\
\hline & $\begin{array}{l}c_{L} \\
c_{R} \\
\end{array}$ & $+2 / 3$ & $\begin{array}{l}1 / 3 \\
4 / 3 \\
\end{array}$ & $1.29_{-0.11}^{+0.05} \mathrm{GeV} / c^{2}$ \\
\hline & $\begin{array}{l}s_{L} \\
s_{R}\end{array}$ & $-1 / 3$ & $\begin{array}{c}1 / 3 \\
-2 / 3 \\
\end{array}$ & $100_{-20}^{+30} \mathrm{MeV} / c^{2}$ \\
\hline & $\begin{array}{l}t_{L} \\
t_{R}\end{array}$ & $+2 / 3$ & $\begin{array}{l}1 / 3 \\
4 / 3\end{array}$ & $172.9 \pm 0.6 \pm 0.9 \mathrm{GeV} / c^{2}$ \\
\hline & $\begin{array}{l}b_{L} \\
b_{R}\end{array}$ & $-1 / 3$ & $\begin{array}{c}1 / 3 \\
-2 / 3 \\
\end{array}$ & $4.19_{-0.06}^{+0.18} \mathrm{GeV} / c^{2}$ \\
\hline \multirow{6}{*}{ Leptons } & $\nu_{e}$ & 0 & -1 & $<2 \mathrm{eV} / c^{2}$ \\
\hline & $\begin{array}{l}e_{L} \\
e_{R}\end{array}$ & -1 & $\begin{array}{l}-1 \\
-2\end{array}$ & $0.511 \mathrm{MeV} / c^{2}$ \\
\hline & $\nu_{\mu}$ & 0 & -1 & $<0.19 \mathrm{MeV} / c^{2}$ \\
\hline & $\begin{array}{l}\mu_{L} \\
\mu_{R} \\
\end{array}$ & -1 & $\begin{array}{l}-1 \\
-2 \\
\end{array}$ & $106 \mathrm{MeV} / c^{2}$ \\
\hline & $\nu_{\tau}$ & 0 & -1 & $<18.2 \mathrm{MeV} / c^{2}$ \\
\hline & $\begin{array}{l}\tau_{L} \\
\tau_{R}\end{array}$ & -1 & $\begin{array}{l}-1 \\
-2 \\
\end{array}$ & $1.78 \mathrm{GeV} / c^{2}$ \\
\hline
\end{tabular}

Table 1.1: Fundamental properties of fermions.

Quarks have an additional quantum number called color charge, which is three types $(r, g$ and $b)$. Color charge is not seen in nature and therefore the colorless composite particle can only exist. The colorless composite particle can be made with two ways: 
bound state of three quarks called baryons such as a proton $\left(u_{r} u_{g} d_{b}\right)$, or bound state of one quark and one antiquark called mesons such as a pion $\left(u_{r} \bar{d}_{r}\right)$.

The interaction between fermions is mediated by spin $s=1$ bosons. The photon $(\gamma)$ carry electromagnetic force, the $W^{ \pm}$and $Z$ bosons carry the weak force, and the gluons $(g)$ carry the strong force. The photon is massless, while the $W^{ \pm}$and $Z$ bosons are massive particles. In the Standard Model, the $W^{ \pm}$and $Z$ bosons acquire the mass as a result of the electroweak symmetry breaking through the Higgs mechanism. The gluon is the massless bi-colored particle and influences only quarks. The properties of bosons are summarized in Table 1.2.

\begin{tabular}{c|c|c|c|c}
\hline \hline Particle & Force & Electric charge $Q$ & Hyper charge $Y$ & Mass \\
\hline$\gamma$ & Electromagnetic & 0 & 0 & 0 \\
\hline$W^{-}\left(W^{+}\right)$ & Weak(charged) & $-1(+1)$ & $-1(+1)$ & $80.399 \mathrm{GeV} / c^{2}$ \\
\hline$Z$ & Weak(neutral) & 0 & 0 & $91.188 \mathrm{GeV} / c^{2}$ \\
\hline$g$ & Strong & 0 & 0 & 0 \\
\hline \hline
\end{tabular}

Table 1.2: Fundamental properties of bosons.

The Standard Model has been successful in describing interactions of the particles described above, all of which have been discovered experimentally. In addition, many of the predicted properties of these particles have been confirmed, some to a high degree of precision. However, in order for the symmetry described in Equation 1.1 to be exact, the fermions and the $W$ and $Z$ bosons would have to be massless. In order for the Standard Model to be compatible with the large masses of the $W$ and $Z$ bosons and thus the large division between the effective weak coupling constant (the Fermi constant) and the electromagnetic coupling constant (the fine structure constant), spontaneous symmetry breaking must occur. This symmetry breaking would additionally be responsible for the mass hierarchy observed in the fermions. This Electroweak Symmetry Breaking (EWSB) is accomplished by the introduction of a scalar field known as the Higgs field [11]. The existence of a massive scalar particle, the Higgs boson, would be associated with the Higgs field.

The existence of the Higgs boson has yet to be confirmed experimentally, and remains one of the most important tasks for the field of high energy physics. Direct searches for the Standard Model Higgs boson at the CERN Large Electron Positron (LEP) collider have set a lower bound on its mass of $M_{H}>114.4 \mathrm{GeV} / \mathrm{c}^{2}$ at the $95 \%$ confidence level [12]. Also, direct search at the Tevatron excluded the mass range between 156 and $177 \mathrm{GeV} / c^{2}$ at $95 \%$ confidence level [13].

In addition, indirect bounds on the mass of the Higgs boson can be set from precision measurements of the top quark and $W$ boson masses, as these quantities are sensitive to $\ln M_{H}$ through radiative corrections. Using measurements of the top quark mass and the precision electroweak measurements made at the LEP, SLD, CDF and D $\varnothing$, the constraints on the Standard Model Higgs bosons are [14] 


$$
\begin{aligned}
& M_{H}=92_{-26}^{+34} \mathrm{GeV} / c^{2} \\
& M_{H}<161 \mathrm{GeV} / c^{2} \text { at } 95 \% \text { C.L.. }
\end{aligned}
$$

\subsection{The Spin Quantum Number}

The concept of spin was first introduced in 1924 by Wolfgang Pauli, although at the time he did not use the term "spin". It was introduced as an internal degree of freedom of the electron, in order to explain observations in atomic physics, but it soon became apparent that spin is an important property of all particles. Although the spin of a particle is an internal property and does not correspond to a physical rotation, it was named spin because it does have some properties that are similar to a physical rotation - for example, it adds to a particle's total angular momentum, and causes a charged particle to interact with a magnetic field.

Leptons and quarks have a total value for their spin of $\frac{1}{2}$. When measuring spin experimentally, a particular axis called the "spin quantization axis" must be chosen. The spin is measured along that axis in the rest frame of the particle and can have one of two values. If a top quark's spin is oriented in the same direction as the quantization axis, it has spin

$s=+\frac{1}{2}$, and if it is oriented opposite the direction of the axis, it has spin $s=-\frac{1}{2}$. The choice of the quantization axis is also referred to as a choice of basis, and there are three common basis for measuring top quark spin in collider experiments:

- Beamline Basis: the spin quantization axis is the direction of the incident colliding particles in the $t \bar{t}$ rest frame.

- Helicity Basis: the spin quantization axis is the direction of motion of the top quark in the $t \bar{t}$ rest frame.

- Off-Diagonal Basis: a hybrid basis where the spin quantization axis is in between the direction of the axis in the helicity basis and the direction of the axis in the beamline basis, with the exact choice of axis dependent on the kinematics of the event [15].

\subsection{The Top Quark}

Since the discovery of the bottom quark in 1977, as all other quarks and leptons have a same family partner, the existence of the top quark was anticipated. The existence of the top quark was inferred for several reasons. For one, the renormalizability of the Standard Model requires that the sum of electric charges of all left-handed fermions must equal zero. This condition is only satisfied with the existence of a sixth quark with an electric charge of $+2 / 3$. In addition, the precise measurements involving the isospin of the $b$-quark can be made at $e^{+} e^{-}$colliders, which can be used to exclude the possibility of the $b$-quark being a member of a singlet [16]. 
The experimental discovery of the top quark took much longer than originally anticipated because the top quark was not expected to be so heavy. The top $(t)$ quark was observed at Fermilab in 1995 by the CDF and D $\varnothing$ collaborations [1,2]. By the end of the 1992-1996 collider run (Run I), combined datasets from both experiments of $\sim 100 \mathrm{pb}^{-1}$ provided a measurement of the top quark mass of $M_{t}=178.0 \pm 4.3 \mathrm{GeV} / c^{2}$ [17].

\subsection{Top Quark Production}

Top quark has a large mass. Therefore, the top quarks can currently be produced at two accelerators in the world: the Tevatron accelerator and the now operational and running LHC accelerator. At the Tevatron, the top quark is produced predominately in top antitop pairs via the strong interaction. There are two different mechanisms on the top quark pair production. They are the quark-antiquark annihilation process $(q \bar{q} \rightarrow t \bar{t})$ and the gluon fusion process $(g g \rightarrow t \bar{t})$. At a center of mass energy $\sqrt{s}$ of $1.96 \mathrm{TeV}$, the processes $q \bar{q} \rightarrow t \bar{t}$ and $g g \rightarrow t \bar{t}$ occur approximately $85 \% \pm 5 \%$ and $15 \% \pm 5 \%$ of the time, respectively [18]. The leading order diagrams for two processes are shown in Figure 1.1 and 1.2.

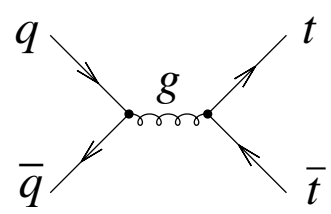

Figure 1.1: Leading-order production diagram for $q \bar{q} \rightarrow t \bar{t}$.

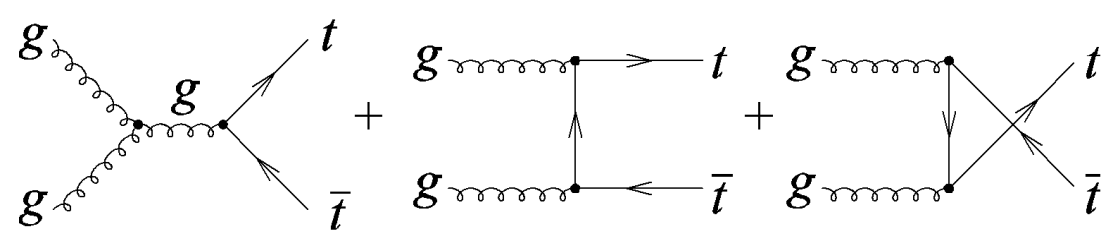

Figure 1.2: Leading-order production diagrams for $g g \rightarrow t \bar{t}$.

The top quark pair production via the $q \bar{q}$ annihilation has a different spin state from that produced via the gluon fusion. When top quark pairs are produced near kinematic threshold (approximately $345 \mathrm{GeV}$ ), the pairs produced via $q \bar{q}$ annihilation and gluon fusion are in the following total angular momentum states, respectively (See Appendix A):

$$
\begin{aligned}
& q \bar{q}: \quad J=1, J_{z}= \pm 1, \\
& g g: \quad J=0, J_{z}=0,
\end{aligned}
$$

where $z$ denotes the initial parton direction.

Therefore, in the case of $q \bar{q}$ annihilation, the top quark and the antitop quark have the aligned spin on the beam axis, while they have the opposite spin on any axis in the case of 
gluon fusion. Figure 1.3 shows an example of the spin configurations of the gluon fusion and $q \bar{q}$ annihilation at a threshold production.
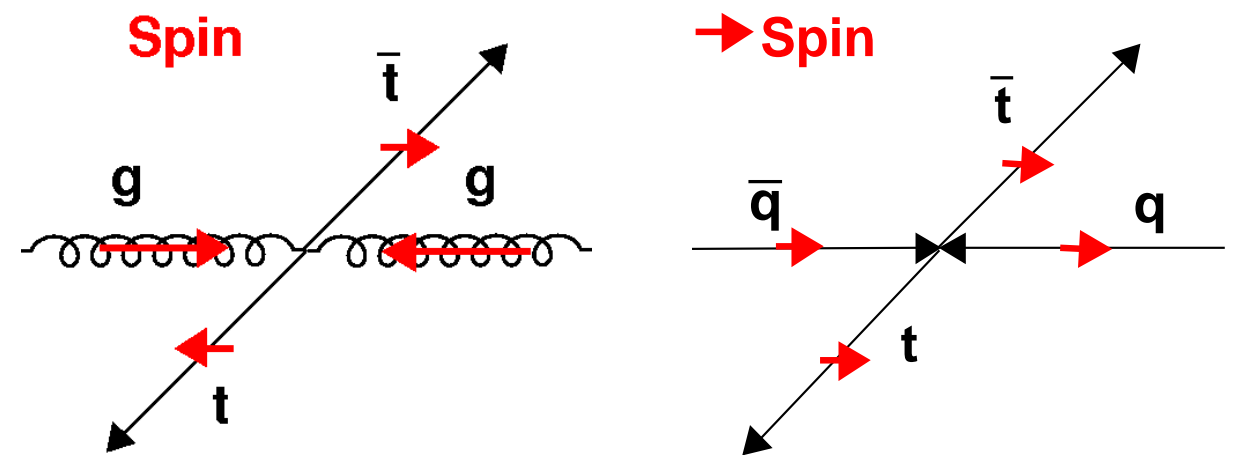

Figure 1.3: The spin configurations of the gluon fusion (left) and the $q \bar{q}$ annihilation (right) subprocesses.

In the actual $p \bar{p}$ collisions, top quark pairs can be produced at center-of-mass energies significantly above threshold. Then, for $q \bar{q} \rightarrow t \bar{t}$ process, for top and antitop quark spin states, $(\uparrow \downarrow)$ and $(\downarrow \uparrow)$ dominate, but a little bit the $(\uparrow \uparrow)$ and $(\downarrow \downarrow)$ spin configuration exist in the beamline basis. On the other hand, for $g g \rightarrow t \bar{t}$ process, the unlike spin configuration between top and antitop quark is mitigated so that top quark pairs slightly anti-correlated spins in the beamline basis.

The theoretical prediction of the $t \bar{t}$ production cross section at next-to-leading order (NLO) is $\sigma^{N L O}(p \bar{p} \rightarrow t \bar{t} X)=6.7_{-0.9}^{+0.7} \mathrm{pb}$ at $M_{t}=175 \mathrm{GeV} / c^{2}$ [18]. Figure 1.4 shows the NLO calculation of $\sigma(p \bar{p} \rightarrow t \bar{t} X)$ for $p \bar{p}$ collisions at $\sqrt{s}=1.96 \mathrm{TeV}$ as a function of the top quark mass.

\subsection{Top Quark Decay}

The top quarks decay almost completely in to a $W$ boson and a $b$-quark. Other decay channels are permitted in the Standard Model, but are heavily suppressed by factors of $\left|V_{t s}\right|^{2} /\left|V_{t b}\right|^{2} \sim 10^{-3}$ and $\left|V_{t d}\right|^{2} /\left|V_{t b}\right|^{2} \sim 5 \times 10^{-4}$, where $V_{i j}$ is the element of the CabibboKobayashi-Masukawa (CKM) weak-mixing matrix [10]. The large mass of the top quark results in a very rapid decay with a mean lifetime of $\tau_{t} \sim 10^{-24} \mathrm{~s}$. As this is shorter than the time scale required for quarks to form bound states (or "hadronize"), the top quark essentially decays as a "free" quark. Therefore, top quark retain its original polarization at the production until decay, and due to the decay via parity violating weak interaction, the information of the parent top polarization is transferred to decay products.

The $b$-quark resulting from the decay will then proceed to hadronize and manifest itself in the detector as a jet, or a collimated stream of hadrons. The $W$ boson will decay rapidly into either a pair of quarks or a pair of charged lepton and a neutrino. Thus, for the case of a $t \bar{t}$ pair production and decay, there are six objects: two $b$-quarks and two decay products from each of the $W$ boson. Figure 1.5 shows the tree level diagram of $t \bar{t}$ production and decay in the subprocess $q \bar{q} \rightarrow t \bar{t}$. 


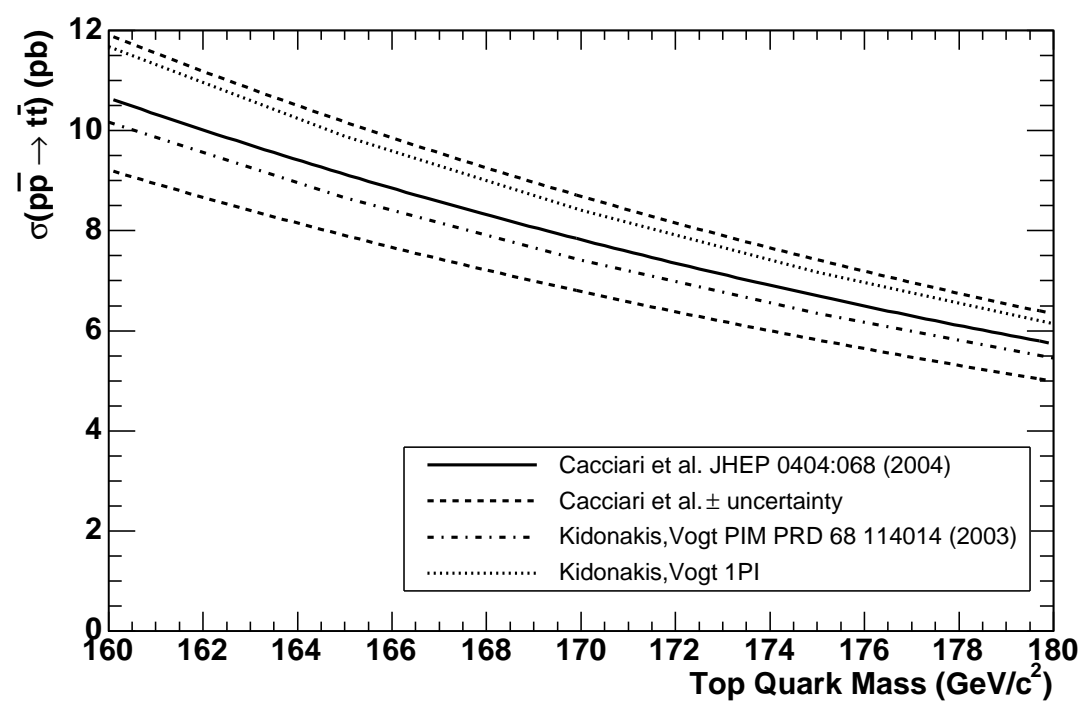

Figure 1.4: NLO calculations of $\sigma(p \bar{p} \rightarrow t \bar{t} X)$ [18] for $p \bar{p}$ collisions at $\sqrt{s}=1.96 \mathrm{TeV}$ as a function of the top quark mass.

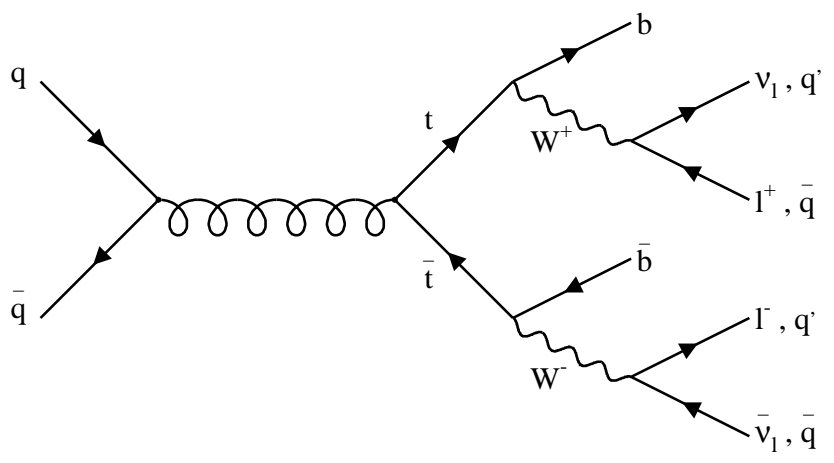

Figure 1.5: Tree level $q \bar{q} \rightarrow t \bar{t}$ production and decay. 
It is the decay mode of the $W$ bosons that defines the decay channels of the $t \bar{t}$ system used in its experimental study. These decay channels are classified as:

- All-hadronic channel, where both $W$ bosons decay to quark-antiquark pairs, resulting in a final state having an experimental signature of six jets. This decay mode carries the largest branching ratio of $46 \%$, but suffers from the largest amount of irreducible QCD background.

- Lepton+jets channel, where one $W$ decays to a lepton-neutrino pair and the other to quarks, resulting in an experimental signature of a high momentum lepton, four jets, and a missing transverse energy associated with the neutrino. Due to the difficulty of identifying $\tau$ leptons at a hadron collider, only leptonic states with an electron or muon in the final state are considered. This channel carries a branching ratio of $30 \%$.

- Dilepton channel, where both $W$ bosons decay to leptons, resulting in an experimental signature of two high momentum leptons, two jets, and large missing transverse energy associated with two neutrinos. As with the lepton+jets channel, only leptonic states with an electron or muon in the final state are considered. This channel carries a branching ratio of $4 \%$. The remaining $20 \%$ of $t \bar{t}$ decays involve the production of a lepton that does not decay to an e or $\mu$. While measurements in this so-called " $\tau+X$ " channel are possible, they do not afford nearly the same precision that any of the other three channels does.

\subsection{Top Quark Mass}

The top quark mass is a fundamental parameter in the Standard Model. Due to its large value, it has a critical influence on the Standard Model calculations than the other quarks. It contributes to higher order (radiative) corrections to electroweak processes, as illustrated in Figure 1.6. It also helps to constrain the mass of Higgs boson via the radiative
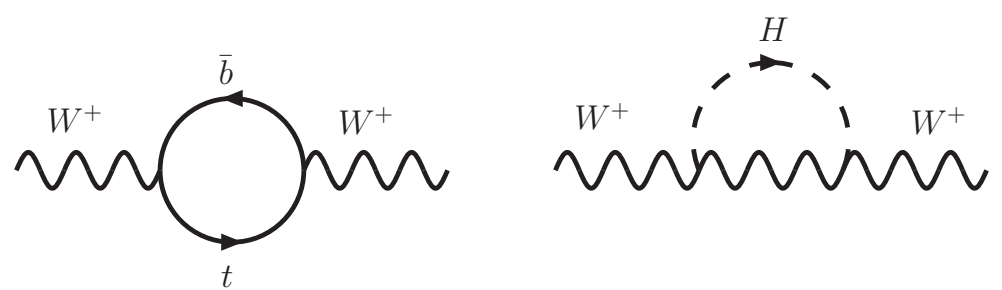

Figure 1.6: Loop diagrams generating corrections to the theoretical $W$ boson mass. On the left is a fermion loop with the top and $b$-quarks. On the right is a Higgs boson loop.

correction to the mass of the $W$ boson. Figure 1.7 shows constraints on the Higgs boson mass using the current best measurements of the $W$ boson and the top quark masses, and a global fit of the Higgs boson mass to several electroweak parameters. As indicated 
in Figure 1.7, the most likely value of the Higgs boson mass is ruled out by the direct searches at LEP. A failure of the Standard Model to properly describe these results may indicate new physics yet to be discovered.

\subsection{Top Quark Spin Correlation}

In the quark pair production by the strong interaction, the quark spins are entangled according to the short distance dynamics of quantum chromodynamics (QCD). The spin state is observable in angular correlations among the quark decay products induced by the $\mathrm{V}$-A nature of the weak interaction, but is typically destroyed by the depolarizing effects of hadronization before the decay can proceed. The top quark is an exception to this rule.

Spin-flip time by hadronizing is described as [19]

$$
O\left(m_{t} / \Lambda_{\mathrm{QCD}}^{2}\right) \simeq(1.3 \mathrm{MeV})^{-1}
$$

On the other hand, the top quark decay width is predicted to be $\Gamma_{t} \simeq 1.42 \mathrm{GeV}$ [20]. Therefore we can find

$$
O\left(m_{t} / \Lambda_{\mathrm{QCD}}^{2}\right) \gg 1 / \Gamma_{t}
$$

and this means the top quark life time is shorter than the fragmentation timescale, cutting off the long distance QCD effects and transmitting the $t \bar{t}$ production configuration to the final state.

In top quark decays in the SM the V-A couplings fix the angular distributions of the decay products according to the polarization of the parent top quark via

$$
\frac{1}{\Gamma} \frac{d \Gamma}{d \cos \theta_{i}}=\frac{1 \pm \alpha_{i} \cos \theta_{i}}{2}
$$

where the positive and negative sign is used for decay products from the top quark which has $\uparrow$ and $\downarrow$ spin state, respectively. $\theta_{i}$ denotes the angle between the quantization axis and the flight direction of the decay particle in the top quark rest frame.

In the V-A weak decay, the spin analyzing power coefficient $\alpha_{i}$ is equal to +1.0 for the charged lepton or down-type quark, -0.41 for the bottom quark, and -0.31 for the neutrino or up-type quark, with the signs reversed for antitop-quark decays [21]. The $t \bar{t}$ spin correlation connects the daughter flight direction on each side of the decay.

The differential cross-section in these variables is

$$
\frac{1}{\sigma} \frac{d^{2} \sigma}{d\left(\cos \theta_{i}\right) d\left(\cos \theta_{j}\right)}=\frac{1+\kappa \alpha_{i} \alpha_{j} \cos \theta_{i} \cos \theta_{j}}{4}
$$

where $i$ and $j$ refer to top-quark and antitop-quark decay products respectively.

$\kappa$ is defined as

$$
\kappa=\frac{N_{\uparrow \downarrow}+N_{\downarrow \uparrow}-N_{\uparrow \uparrow}-N_{\downarrow \downarrow}}{N_{\uparrow \downarrow}+N_{\downarrow \uparrow}+N_{\uparrow \uparrow}+N_{\downarrow \downarrow}}
$$

where $N_{s_{t} s_{\bar{t}}}$ indicate number of $t, \bar{t}$ in $s_{t}, s_{\bar{t}}$ spin state with respect to the quantization axis for the top quark. $\kappa$ is a parameter between -1 and 1 that depend on the quantization 

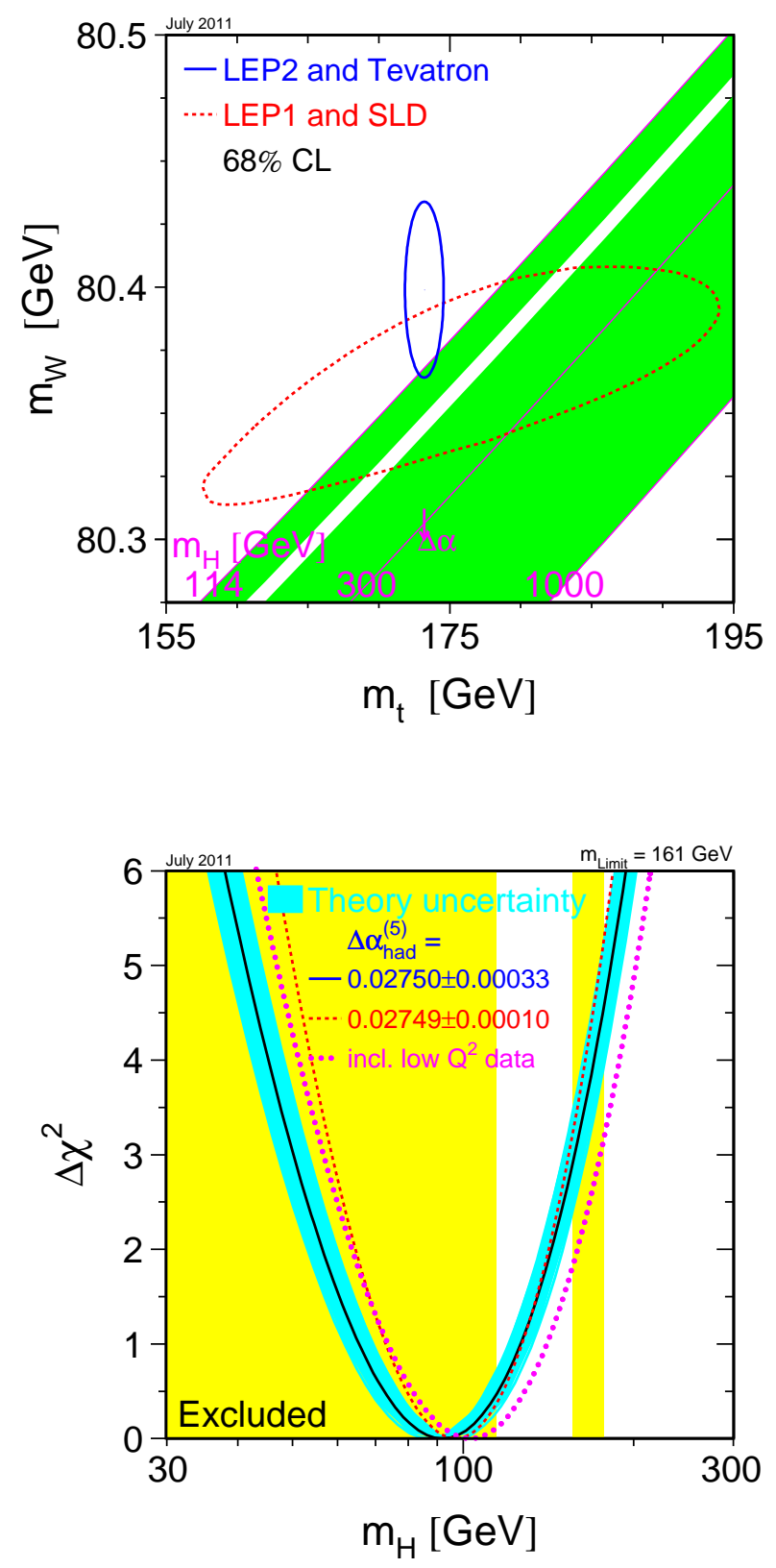

Figure 1.7: Electroweak constraints on the Higgs boson mass using the current best measurements of $W$ boson and top quark mass (top). Global fit of Higgs boson mass to several electroweak parameters (bottom). 
axis used and determines the magnitude of the $t \bar{t}$ spin correlation. $\kappa=+1(-1)$ give full correlated (anticorrelated) spins and $\kappa=0$ corresponds to no spin correlation. Theoretical calculation of $\kappa$ which include next-to-leading order effects predicts $\kappa=0.777$ in the beamline basis, $\kappa=0.782$ in the off-diagonal basis and $\kappa=0.352$ in the helicity basis, at the Tevatron [15].

$t \bar{t}$ pair produced via $q \bar{q}$ annihilation and that produced via $g g$ fusion have different spin states. Therefore, flight direction of the decay particle also depends on the $t \bar{t}$ production mechanism. Fig 1.8 shows two dimensional distributions of $\cos \theta_{+}$and $\cos \theta_{-}$in the beamline basis for the processes $q \bar{q} \rightarrow t \bar{t}$ and $g g \rightarrow t \bar{t}$ exclusively. These distributions are made with Herwig event generator [22] on the assumption of CTEQ5L PDF set [23] and $M_{\text {top }}=172.5 \mathrm{GeV} / \mathrm{c}^{2}$. As we mentioned in the Section $1.4, \kappa$ in the beamline basis for $q \bar{q}$ annihilation is expected to be near the unit, while for $g g$ fusion, $\kappa$ has a negative value as expected. By fitting the distributions to a function $C_{0} \cdot\left(1+\kappa \cos \theta_{+} \cos \theta_{-}\right)$, we found $\kappa=0.958 \pm 0.003$ for $q \bar{q} \rightarrow t \bar{t}$ and $\kappa=0.369 \pm 0.016$ for $g g \rightarrow t \bar{t}$.
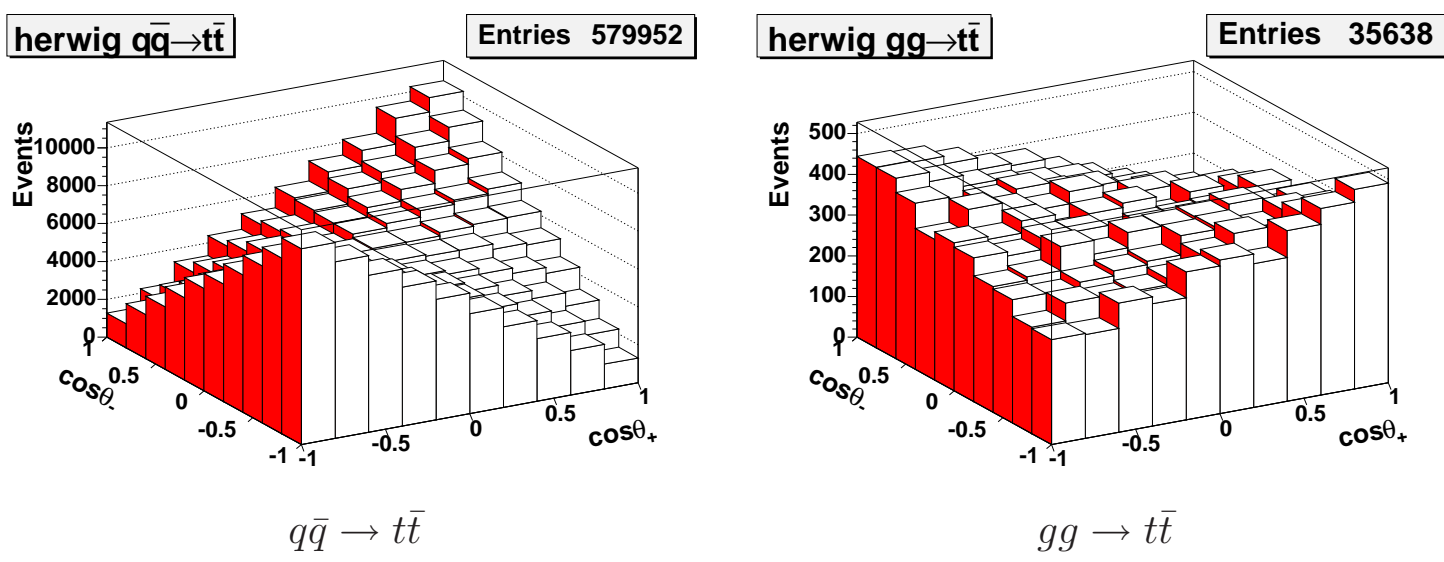

Figure 1.8: The two-dimensional distributions of $\cos \theta_{+}$and $\cos \theta_{-}$in beamline basis for the processes $q \bar{q} \rightarrow t \bar{t}$ and $g g \rightarrow t \bar{t}$.

\section{$1.8 \kappa^{\text {meas }}$, what is measured in this analysis}

As described in Section 1.7, the top quark is expected to decay before losing spin polarization at its production and the charged lepton flight direction in the top rest frame is maximally correlated to the top polarization.

In this measurement, we suppose that $t \bar{t}$ is once produced with the Standard Model spin correlations, i.e. $\kappa \sim 0$.8. However top and/or anti-top polarization might be lost when they decay because of spin flip by hadronization, such as interaction with gluons. Suppose $\kappa^{\text {prod }}$ is spin-spin correlation coefficient of $t \bar{t}$ pair at its production, and $C_{t}\left(C_{\bar{t}}\right)$ denotes a fraction of the top (anti-top) polarization at production which is kept until it decays. Then the differential decay rate equivalent to Equation 1.8 is proportional to

$$
1+C_{t} C_{\bar{t}} \kappa^{\text {prod }} \cos \theta_{+} \cos \theta_{-} .
$$


Here the standard model predicts top quark decays before losing its polarization, i.e. $C_{t}=$ $C_{\bar{t}}=1$.

Substantially, what we measure in this analysis is $C_{t} C_{\bar{t}} \kappa^{\text {prod }}$. If we observe nonzero correlation coefficient $\kappa^{\text {meas }}$ in $t \bar{t}$ production and decay, that consequently indicates a direct evidence that top and anti-top are produced with their spins being correlated and decay as bare quarks before losing their spin polarizations.

\subsection{Motivation of Spin Correlation Measurement}

Measurement of the spin correlation is important not only as a test of the theoretical prediction, but also as a possible factor in helping to understand new physical interactions. Known interactions that can produce top quark pairs are well understood, leading to accurate predictions for the $t \bar{t}$ spin correlation. Any deviation of the measured spin correlation from the theoretical predictions would indicate new top quark production processes. An example of such a process would be $t \bar{t}$ production via Kaluza-Klein graviton in a RandallSundrum model [24], which would alter the spin correlation because unlike the gluon, the graviton has a total spin of 2. In particular, resonant production of such a graviton would produce large changes in the spin correlation as a function of the $t \bar{t}$ invariant mass compared to current theoretical predictions. Precision studies of the spin correlation and its behavior as a function of $t \bar{t}$ mass could provide evidence for or against this model and many other models of new physical process.

A recent CDF measurement found an asymmetry in the direction that the top quark from a $t \bar{t}$ pair travels after it is produced that disagrees with the theoretical prediction by approximately $3 \sigma$ [25]. Many models of new physical process have been proposed to explain this discrepancy, and many of these models also predict that the new interactions will have an effect on the top quark pair spin correlation [26]. A precise measurement

of the spin correlation would differentiate among these models, probing new physical interactions.

\subsection{Previous Measurement of Top Quark Spin Correla- tion}

Measurements of spin correlation has been performed by the CDF, D $\varnothing$ and ATLAS experiments.

\subsubsection{Dilepton channel}

The $\mathrm{D} \varnothing$ experiment reported on two measurement results in beamline basis using $t \bar{t}$ dilepton candidates in data of $5.4 \mathrm{fb}^{-1}$. One of the two results is measured using a matrix element approach with $485 t \bar{t}$ dilepton candidate events. Its result is $\kappa=0.57 \pm 0.31$ (stat+syst) [27]. Another is obtained from differential angular distribution template in lepton decay angles with $441 t \bar{t}$ dilepton candidate events. Its result is $\kappa=0.10 \pm 0.45$ (stat+syst) [28]. 
ATLAS experiment reported on a result in helicity basis using $t \bar{t}$ dilepton candidates in data of $0.7 \mathrm{fb}^{-1}$. Standard Model predicts $\kappa=0.32$ in helicity basis for $t \bar{t}$ spin correlation in $t \bar{t}$ production from proton-proton collisions at a center-of-mass energy of $7 \mathrm{TeV}$. The measurement result at ATLAS experiment yielded $\kappa=0.34 \pm 0.07$ (stat) ${ }_{-0.09}^{+0.13}$ (syst) [29]. This result is consistent with the Standard Model prediction and it indicates the existence of a spin correlation in $t \bar{t}$ events at a level of $3 \sigma$ equivalence.

\subsubsection{Lepton+Jets channel}

The CDF experiment reported on measurement results with $1001 t \bar{t}$ lepton plus jet candidates in data of $5.3 \mathrm{fb}^{-1}$. Its result is $\kappa=0.72 \pm 0.64$ (stat) \pm 0.26 (syst) [30] in beamline basis.

\subsubsection{Combine lepton+Jets events and dilepton events}

The $\mathrm{D} \varnothing$ experiment reported on a result that obtained by combining a result in lepton plus jets events with the result in dilepton events, where both results are obtained using the matrix element approach. Its result is $\kappa=0.66 \pm 0.23$ (stat+syst) in data of $5.3 \mathrm{fb}^{-1}$ [31] in beamline basis. This result indicates the existence of spin correlation in $t \bar{t}$ events at a level of 3.1 standard deviations equivalence.

\subsection{Outline of This Thesis}

In this thesis we measure the strength of the $t \bar{t}$ spin correlation, $\kappa$, from a differential angular distribution of the two decay leptons in the rest frames of their respective $t$ quark and the spin quantization axis. To measure $\kappa$, we use data of $5.1 \mathrm{fb}^{-1}$ collected with the Collider Detector Facility (CDF) at the Fermilab Tevatron between March 2002 and June 2009. Chapter 1 describes the short summary of the Standard Model and the top quark physics. Chapter 2 describes the experimental apparatus of Tevatron accelerator and CDF detector. In Chapter 3, the event reconstruction using information from the detector responses is discussed. Chapter 4 describes the event selection to select the dilepton candidate events, the method of the full kinematical reconstruction, preparation of signal/background templates and the construction of Feldman-Cousins confidence belt from pseudo-experiments. Chapter 5 describes an estimation of systematic uncertainties in our measurement. Chapter 6 describes the measurement result and discussion, and Chapter 7 concludes this analysis. 


\section{Chapter 2}

\section{Experimental Apparatus}

This chapter describes the Fermilab accelerator complex including the Tevatron collider, CDF II detector components, trigger and data acquisition (DAQ) system.

\subsection{The Fermilab Accelerator Complex}

The Tevatron is a circular particle collider in the United States at the Fermilab. The Tevatron was the second most powerful proton-antiproton accelerator in the world before it shut down. The reason why the Tevatron accelerates protons and antiprotons is the following two reasons.

- Synchrotron radiation of protons and antiprotons is much smaller than it of electrons and positrons at high energy in circular accelerators: Although electron-positron collisions are easier to analyze (because electrons are single point-like particles, unlike the composite protons), the large synchrotron radiation of electrons prohibits their use at high energies in circular accelerators. Because synchrotron radiation increases as the inverse of the fourth power of a particle's mass, protons, which have roughly 2000 times the mass of electrons, radiate much less. Protons and antiprotons are currently the only viable alternative to electrons and positrons.

- A collider's design is vastly simplified if it collides particles with their antiparticles, because the particles can travel opposite directions in the same beam-pipe and be bent by the same set of magnets.

The accelerating system consist of a few accelerators(Figure 2.1) where the protons and antiprotons are gradually accelerated up to final energies. The Tevatron is the last in a chain of accelerators. The Tevatron's first physics run, referred to as Run I, occurred from 1992-1996. After a series of upgrades, it began running in 2002 and finished running in 2011.

The detector located at proton-antiproton collision points need to disentangle all the particles that are produced and need to do this every $396 \mathrm{~ns}$, keeping only those events that mark "interesting physics", the anomalies. Not only do we need to understand what type of particles we expect and how often they should be produced, but we also need to know how these particles will interact in different environments so we can build detectors 
in ways that we can distinguish the difference between the types of particle produced. Until we can be fairly confident in how the detector works and its different idiosyncrasies that need to be calibrated and understood, we can not make any interesting connections with what theory tells us.

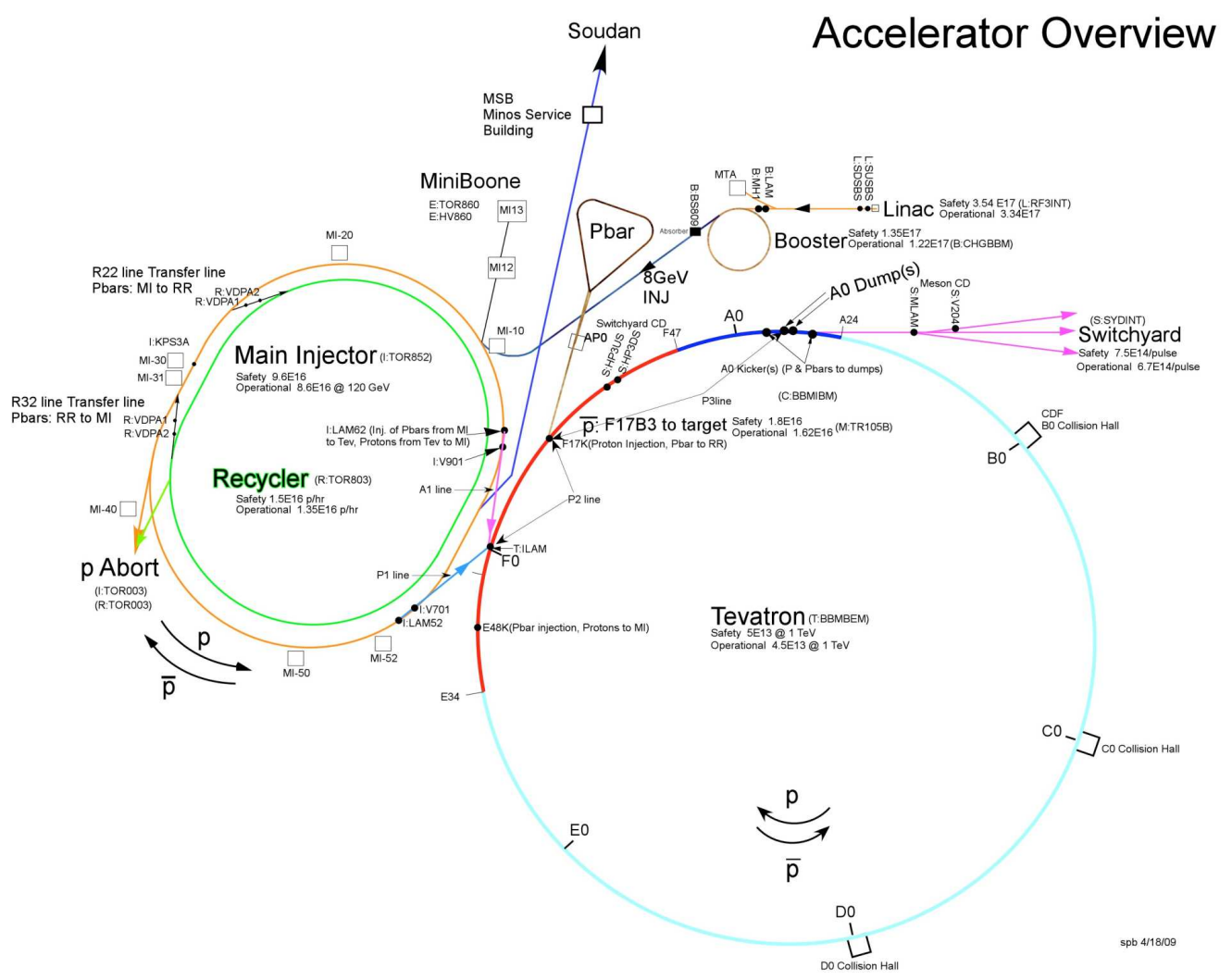

Figure 2.1: A diagram of the Fermilab accelerator chain.

\section{Proton Source}

The protons used in the Tevatron are originally extracted from very pure hydrogen gas. For ease of insertion into the Booster, the particles accelerated are actually $\mathrm{H}^{-}$ions instead of protons. Hydrogen gas is moved between two electrodes and a spark ionizes the hydrogen into electrons and $\mathrm{H}^{+}$ions. The positive ions strike a cathode made of cesium, which has a low work function and thus releases electrons easily, and occasionally pick up two electrons and form $\mathrm{H}^{-}$ions. An electrostatic extractor sends them to the preaccelerator.

\section{Preaccelerator}

The preaccelerator is a Cockcroft-Walton-style [32] electrostatic accelerator. Ions from the proton source are subjected to a potential of $-750 \mathrm{kV}$, thus producing beams of $\mathrm{H}^{-}$ions with an energy of $750 \mathrm{keV}$. The potential is created by the 5 -stage diode voltage multiplier, which converts $75 \mathrm{kV}$ AC to the $-750 \mathrm{kV}$ DC. The extracted $\mathrm{H}^{-}$ions are accelerated to 
$750 \mathrm{keV}$ by passing through the accelerating column. The preaccelerater beam every 66 milliseconds (a $15 \mathrm{~Hz}$ repetition rate) whether beam is being requested or not. The $\mathrm{H}^{-}$ ions are steered and focused by magnets down a transfer line to the Linac.

\section{Linac}

The Linear Accelerator is the next Level of acceleration for the negative charged $\mathrm{H}^{-}$ions. It is abbreviated to Linac. It takes the ions with energy of $750 \mathrm{keV}$ and accelerates them to an energy of $400 \mathrm{MeV}$. The Linac consists of two main sections, the low energy drift tube Linac and the high energy side-coupled cavity Linac. The drift tube Linac (DTL) makes up the first five RF stations. It accelerate the ion beam to $117 \mathrm{meV}$. Beam in the DTL is focused by means of quadrupole magnets located inside the drift tubes, which in turn are located inside the RF cavities. The side-coupled cavity Linac (SCL) has seven side-coupled cavity modules that accelerate the $\mathrm{H}^{-}$ions to $400 \mathrm{meV}$. The beam traveling through the SCL is focused by quadrupoles placed between the accelerating modules (outside of the accelerating cavities).

At the far end of the Linac is a chopper, that electrostatically selects a portion of the Linac beam to be sent along a transfer line to the Booster. The Linac completes fifteen acceleration cycles per second.

\section{Booster}

The Booster is a proton synchrotron, approximately $150 \mathrm{~m}$ in diameter. It takes the $400 \mathrm{MeV} \mathrm{H}^{-}$ions from the Linac and strips the electrons off, which leaves only the proton, and accelerating the protons to $8 \mathrm{GeV}$. It has the same duty cycle as the Linac, $15 \mathrm{~Hz}$. The acceleration is accomplished by 18 ferrite-tuned RF cavities located around the Booster ring. A 100 kW PA ( Power Amplifier) drives each cavity. 96 conventional magnets with a maximum field of $0.7 \mathrm{~T}$ bend the beam into a circular orbit. The Booster is able to hold multiple batches of particles from the Linac at once to increase beam intensities, often storing eleven or twelve batches in its ring.

A special set of magnets handles the injection of incoming $\mathrm{H}^{-}$ions from the Linac. Each magnet bends the beam and focuses the beam either horizontally or vertically. The $\mathrm{H}^{-}$ions and circulating beam passes through the stripping foil, which removes the electrons from most of the $\mathrm{H}^{-}$ions and yields protons. A similar set of magnets steers the beam back into the Booster orbit while removing any leftover $\mathrm{H}^{-}$ions.

A set of fast kicker magnets extracts the proton beam from the Booster. The protons go into a transfer line that leads to the Main Injector.

\section{Main Injector}

The Main Injector is a large proton synchrotron with a diameter of about $1 \mathrm{~km}$. It has two main functions involving the Tevatron: accelerating protons and antiprotons for injection into the Tevatron, and accelerating protons to be sent to the antiproton source.

Main Injector has 18 accelerating cavities. It uses 344 dipole magnets and 208 focusing quadrupole magnets, all conventional water-cooled electromagnets, to steer the proton 
beam. It can accelerate protons to $120 \mathrm{GeV}$ or $150 \mathrm{GeV}$. When used to stack antiprotons, the final energies is $120 \mathrm{GeV}$. When used to inject into the Tevatron, the final beam energy is $150 \mathrm{GeV}$.

As well as accepting protons from Booster, the Main Injector can accept antiprotons from the Antiproton Source. The Main Injector can accelerate beam as fast as every 2.2 seconds.

\section{Antiproton Source}

The antiproton source produces antiprotons for use in Tevatron collisions. The Main Injector sends $120 \mathrm{GeV}$ protons down a transfer line to a nickel target. (Nickel was chosen because it can absorb more heat without melting than other metals.) Antiprotons are among the products resulting from this collision; they are selected by an electromagnetic selector and focused down a transfer line to the Debuncher. Studies have shown that $120 \mathrm{GeV}$ is the optimal energy for antiproton production; at this energy, approximately one antiproton is collected per $10^{5}$ protons sent to the antiproton source. The resultant antiprotons have an average energy of about $8 \mathrm{GeV}$.

\section{Debuncher}

The Debuncher is not an accelerator but a triangular storage ring. Its main purpose is to "debunch" the particle beam, removing its RF bunch structure. Magnets in the Debuncher decrease the momentum spread of the antiprotons by rotating them in phase space, trading momentum spread for time spread. This results in a beam of particles that have no RF bunch structure but have roughly uniform momentum. Antiprotons remain in the Debuncher until the next batch of protons is sent to the antiproton target, at which point the antiprotons are sent to the Accumulator.

\section{Accumulator}

The Accumulator lies in the same tunnel as the Debuncher. It is a long term antiproton storage ring, designed to store antiprotons with minimal losses for days. Antiprotons from the Debuncher are manipulated by RF systems in the Accumulator to fill a stable region of phase space, known as the core. The core is kept as small as possible to minimize the momentum spread of the antiprotons; a smaller beam gives a higher luminosity upon injection into the Tevatron.

While the antiprotons stay in the Accumulator, they are reduced in transverse momentum through a process called stochastic cooling [33]. This procedure measures the momentum spread of a group of antiprotons and sends a signal across the ring to corrector magnets, which adjust their fields for each group of particles to reduce the momentum spread of those particles. This results in denser antiproton beams injected into the Tevatron, increasing the resulting luminosity.

Extraction from the Accumulator requires the antiprotons to be collected into bunches again. Adiabatic activation of RF stations causes a portion of the beam to be collected 
into bunches, which are then transferred back to the Main Injector, decelerated to $8 \mathrm{GeV}$, and injected into the Recycler.

\section{Recycler}

When the Accumulator reaches its maximum optimal capacity, its antiprotons are passed into the Recycler, a ring of permanent magnets in the same tunnel as the Main Injector. This storage ring keeps antiprotons at $8 \mathrm{GeV}$, collecting them until the Tevatron is ready for injection. In the Recycler, antiprotons are cooled further using a process called electron cooling [34], in which a beam of electrons is accelerated to the same energy as the antiprotons and runs alongside them. Transverse momentum from the antiproton beam is passed to the much lighter electrons, causing the antiprotons to lose transverse momentum, making the beam smaller. Antiprotons are injected from the Recycler to the Main Injector, which accelerates them to $150 \mathrm{GeV}$ for injection into the Tevatron.

\section{Tevatron}

The Tevatron is the largest of the Fermilab accelerators, with a circumference of approximately $1 \mathrm{~km}$. It is a circular synchrotron and keeps both protons and antiprotons in the same beampipe, revolving in opposite directions. The Tevatron accept both protons and antiprotons from Main Injector and accelerate them from $150 \mathrm{GeV}$ to $980 \mathrm{GeV}$.

Electrostatic separators produce a strong electric field that keeps the two beams from touching except at the collision point. The beam is steered by 774 superconducting dipole magnets and 240 quadrupole magnets with a maximum magnetic field of $4.2 \mathrm{~T}$. They are cooled by liquid helium to $4.2 \mathrm{~K}$, at which point the niobium-titanium alloy in the magnets becomes superconducting.

The Tevatron holds 36 bunches each of protons and antiprotons. The process of injecting particles into the machine, accelerating them, and initiating collisions, referred to as a shot, starts with injection of protons, one bunch at a time, at $150 \mathrm{GeV}$ from the Main Injector. The antiprotons are injected four bunches at a time from the Recycler through the Main Injector. RF cavities accelerate the beams to $980 \mathrm{GeV}$, and then some electrostatic separators switch polarity to cause the beams to collide at two points. Each collision point lies at the heart of a particle detector: one named D0 and the other named the Collider Detector at Fermilab (CDF).

\subsection{The CDF II Detector}

The CDF II detector is a general purpose detector designed to study $p \bar{p}$ collisions at the Tevatron. The detector components are arranged in cylindrical shape. The position of the sub-detectors are described in cylindrical coordinates $(r, \phi, z)$ with fixing the origin to the geometric center of the detector. The $\phi=0$ is parallel to the ground and points out of the Tevatron ring. The $\mathrm{z}$-axis points along the beam in the direction of the protons.

The outgoing particles are described in spherical coordinates. The $\mathrm{z}$-axis is replaced by the polar angle $\theta$. In the event reconstruction, the transverse momentum of particles 
$p_{T}=|\boldsymbol{p}| \sin \theta$ is measured in the transverse plane, and their direction is given by the pseudorapidity $\eta$. The pseudo-rapidity is defined as

$$
\eta=-\ln \tan \left(\frac{\theta}{2}\right)
$$

which is a good approximation at high energies $\left(p_{T} \gg m\right)$ to the rapidity

$$
y=\frac{1}{2} \ln \left(\frac{E+p_{z}}{E-p_{z}}\right)
$$

where $E$ is the particle's energy and $p_{z}$ is its momentum along the z-axis.

A solid cutaway view of the CDF II detector is shown in Figure 2.2. In the center of the detector, the charged particle tracking system is enclosed by a superconducting solenoid. Outside the solenoid is the calorimeter system which is surrounded by the muon detectors.

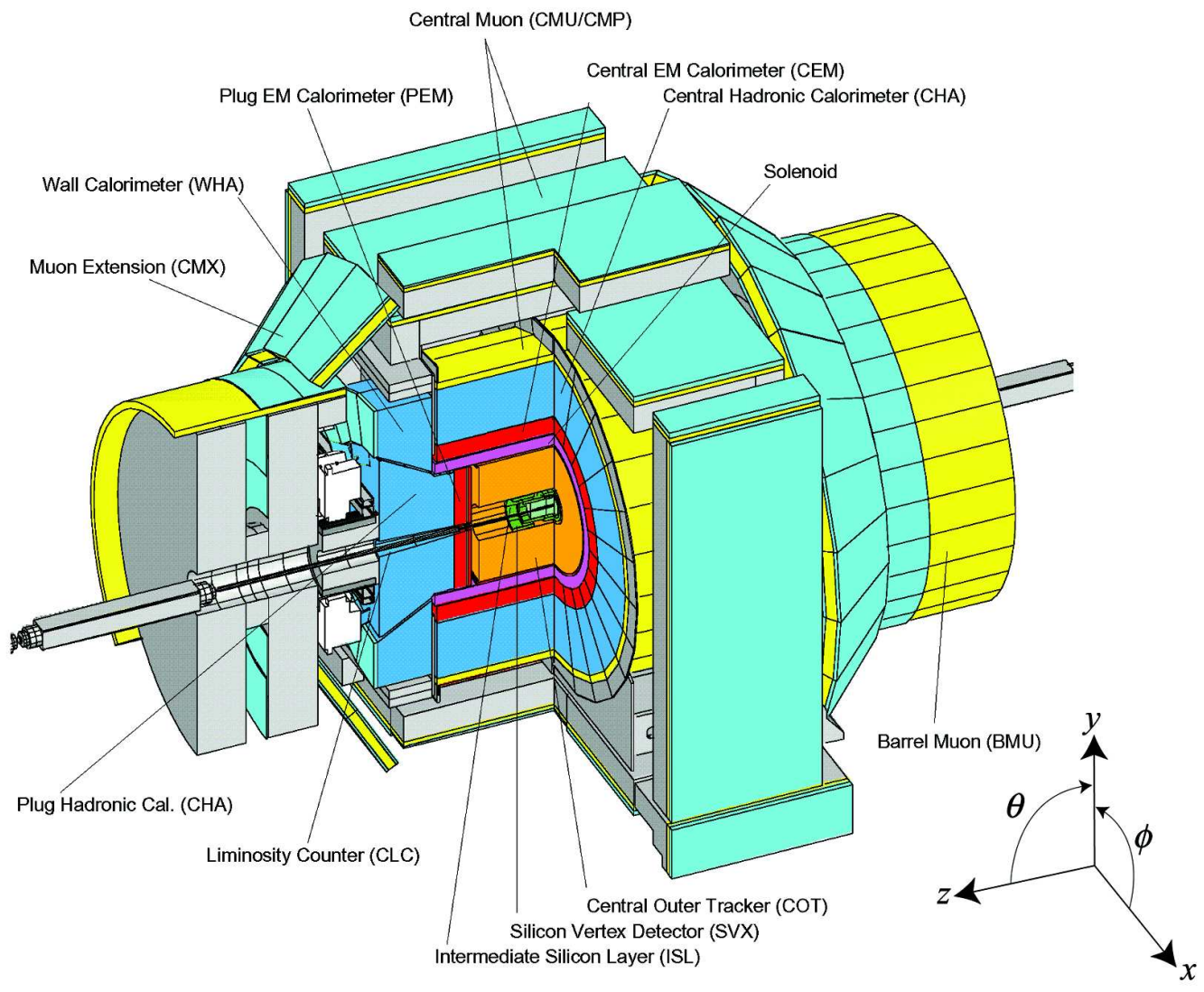

Figure 2.2: Isometric view of the CDF II detector.

\subsubsection{Tracking System}

The innermost part of the CDF II detector is the tracking system which is composed of multi-layer silicon microstrip detectors, an open-cell wire drift chamber, and a superconducting solenoid magnet. The coverage of these detectors are illustrated in Figure 2.3. It is 
used to reconstruct trajectories of charged particles and precisely measure their momenta. The reconstructed particle trajectories are called tracks. Good resolution is required to detect displaced secondary vertices, which is a key to detect $B$ hadrons.

\section{CDF Tracking Volume}

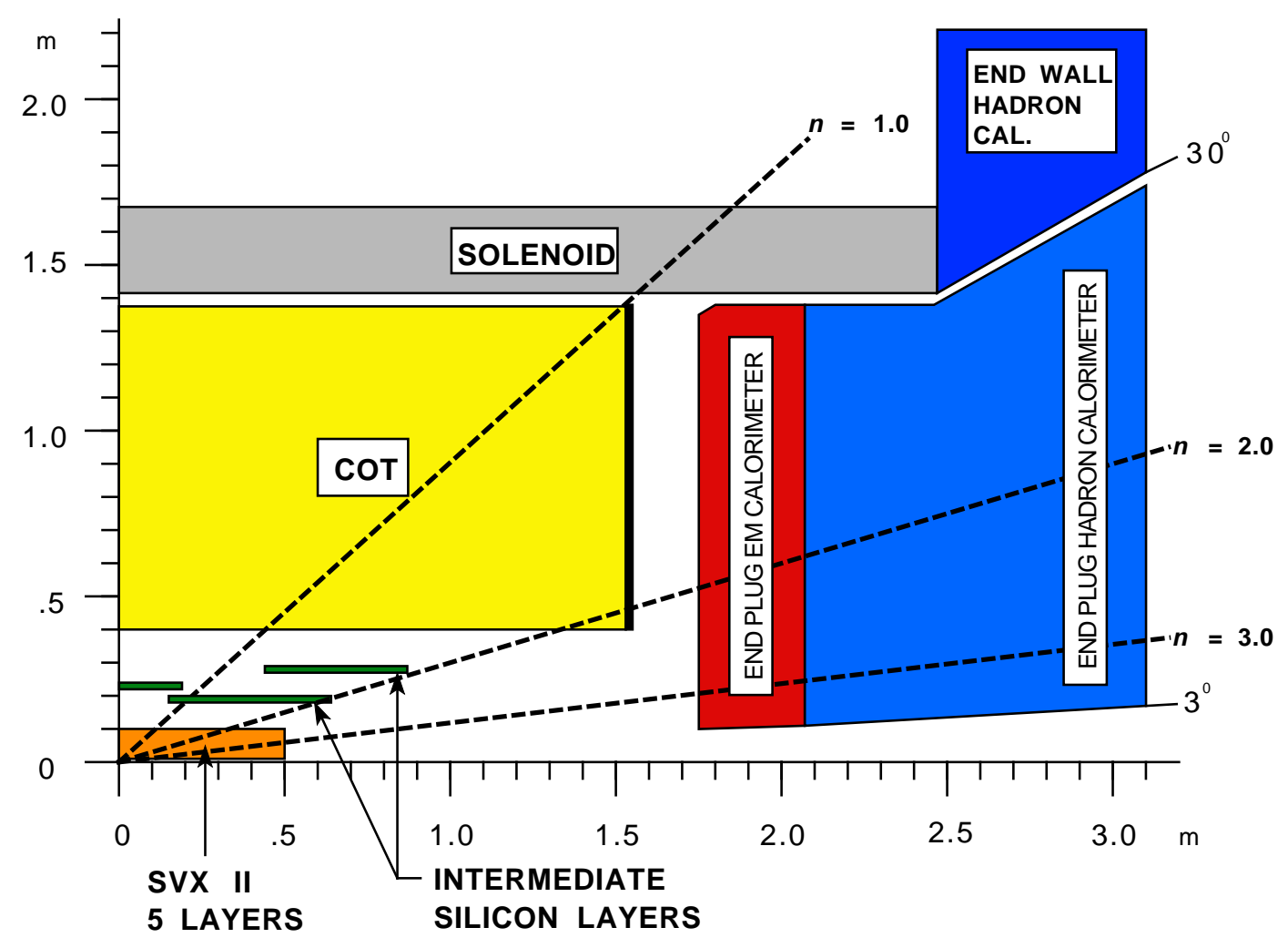

Figure 2.3: The CDF tracking system.

\section{Silicon Detectors}

The silicon detector consists of three parts: Layer 00 (L00) [35], the Silicon Vertex Detector (SVXII) [36], and the Intermediate Silicon Layers (ISL) [37], as shown in Figure 2.4. The detector offers full tracking coverage for $|\eta|<2.0$ as shown in Figure 2.4 (left).

All the three sub-detectors are constructed from wafers of n-type silicon with thin strips $(\sim 10 \mu \mathrm{m})$ doped with p-type silicon (n-type in addition for SVXII sensors). The reverse bias voltage extends the depletion region from the $\mathrm{p}-\mathrm{n}$ junction. When a charged particle passes through the depleted region, it ionizes the silicon wafer creating electron and hole pairs. The voltage moves electrons to one side of the sensor, the holes to the other side. Then, collected charge is read out by ASIC chips mounted at the end of the sensors. The spacial resolution is varying depending on each silicon sub-detector, since pitches are ranging from $25 \mu \mathrm{m}$ to more than $100 \mu \mathrm{m}$.

L00: The L00, the innermost silicon detector, consists of one layer of single-sided silicon 


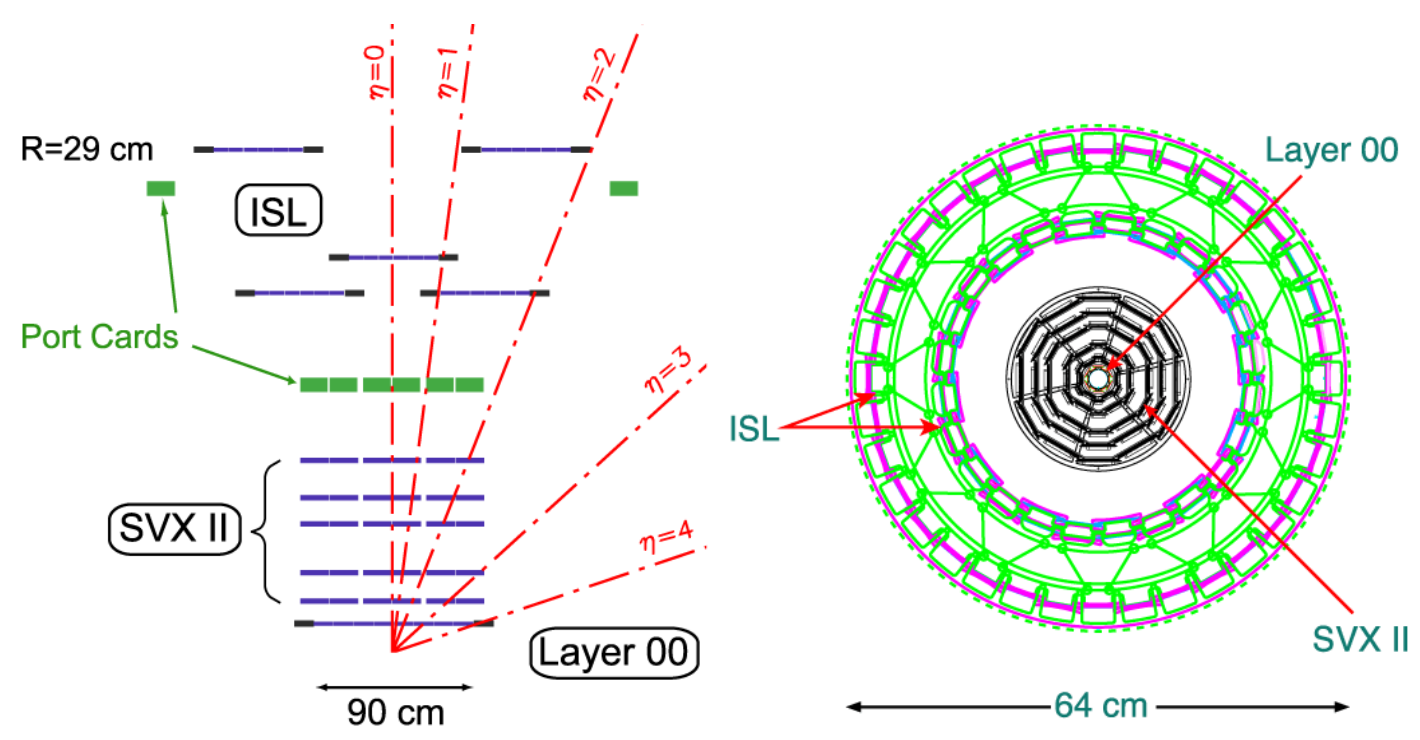

Figure 2.4: The CDF silicon detector. Left: the coverage of the silicon detector in $\mathrm{r}-\mathrm{z}$ plane. Right: Configuration of the silicon detector in $\mathrm{r}-\phi$ plane.

attached directly to the beampipe, only at $\sim 1.5 \mathrm{~cm}$ radius. Its purpose is to improve the resolution of the track impact parameter and position of secondary vertices.

SVXII: The SVXII is the main part of the silicon detector, which consists of five concentric layers of double-sided silicon. These layers are placed at radii from $2.4 \mathrm{~cm}$ to $10.7 \mathrm{~cm}$. The hit information of the SVXII provides high resolution tracking information and is especially useful for reconstructing displaced secondary vertices.

ISL: The ISL is the outermost silicon detector, which consists of a single layer at $|\eta|<$ 1.0 (at a radius of $22 \mathrm{~cm}$ ) and two layers at $1.0 \leq|\eta| \leq 2.0$ (at a radius of $20 \mathrm{~cm}$ and $29 \mathrm{~cm}$ ). This detector helps the connection of tracks between the Central Outer Tracker and the SVXII. This improves the track resolution and the performance of forward tracking in $|\eta| \leq 2.0$.

\section{Central Outer Tracker (COT)}

The COT is a cylindrical drift chamber that sits directly outside of the silicon detectors in the central region $(|\eta|<1.0)$ [38]. The chamber consists of eight cylindrical radial sections ("superlayers") of $310 \mathrm{~cm}$ long cells at radii between 40 and $132 \mathrm{~cm}$ from the detector center. The eight superlayers are placed in alternating axial and stereo sections: wires in axial superlayers run parallel to the $z$-axis, while wires in stereo superlayers are strung at \pm 2 degree angles with respect to the $z$-axis. The number of cells in the superlayer increases radially outwards, the innermost superlayer consists of 168 cells and the outermost one consists of 480 cells. Each cell contains twelve sense wires and thirteen potential wires placed alternately. The chambers are filled with a mixture of argon and ethane gasses, which is chosen to have a uniform drift velocity across the cell volume. 
Along a charged particle passing through the chamber, the gasses in the chamber are ionized. The electrons are drifted forward the sense wire by the electric field, and then they create an avalanche of charges which induce a pulse onto the sense wire. The position resolution of the COT is about $140 \mu \mathrm{m}$ per cell, and the transverse momentum resolution is $\frac{\sigma_{p_{T}}}{p_{T}^{2}}=0.0015[\mathrm{GeV} / c]^{-1}$.

\section{Time-of-Flight System (TOF)}

The TOF detector [39] is incorporated into the CDF detector in order to identify particles up to $1.5 \mathrm{GeV} / c$. By measuring the time it takes for a collision product to reach the TOF, we can separate particles which have different masses, such as $\pi^{ \pm}$and $K^{ \pm}$. This detector is located between the COT and the superconducting solenoid at a radius of $140 \mathrm{~cm}$ with a coverage in $|\eta| \leq 1.0$. In this analysis, we do not use for particles discrimination but use for the event veto coming from cosmic rays.

\section{Superconducting Solenoid}

The superconducting solenoid operated at a current of about 4650 A produces an uniform magnetic field of $1.4 \mathrm{~T}$ parallel to the $z$-axis. The conductor is made of Al-stabilized NbTi. This strong magnetic field bends the trajectory of high- $p_{T}$ charged particles, allowing us to reconstruct their momentum using the tracking system.

\subsubsection{Calorimeters}

The CDF calorimeters measure the energy of both charged and neutral particles. They are sampling scintillator calorimeters segmented into towers having a geometry projected to the detector center. The calorimeter system consists of electromagnetic (EM) and hadronic calorimeters, covering $2 \pi$ in azimuth over the range $|\eta|<3$.6. The cross section of the CDF calorimeter system is shown in Figure 2.5.

The EM calorimeter system consists of two sections: the central EM calorimeter [40] $(|\eta|<1.1)$ and the plug EM calorimeter [41] $(1.1<|\eta|<3.6)$. Both sections include the main calorimeter, which mainly measures the energy of particles, and the shower maximum detector, which helps to improve the position resolution of the calorimeter clusters. The hadronic calorimeter system consists of three sections: the central hadronic calorimeter $(|\eta|<0.9)$, the wall hadronic calorimeter $(0.7<|\eta|<1.3)$, and the plug hadronic calorimeter $(1.3<|\eta|<3.6)$.

\section{Central Electromagnetic Calorimeter (CEM)}

The CEM is segmented into 24 towers in $\phi$ and 10 towers in $\eta$. The single CEM wedge is shown in Figure 2.6. It is a lead-scintillator sampling calorimeter having a radiation length $\left(X_{0}\right) 18 X_{0}$. The energy resolution of the CEM is

$$
\frac{\sigma_{E}}{E}=\frac{13.5 \%}{\sqrt{E(\mathrm{GeV})}} \oplus 2 \%
$$

where the notation $\oplus$ sums the constant and stochastic term in quadrature. 


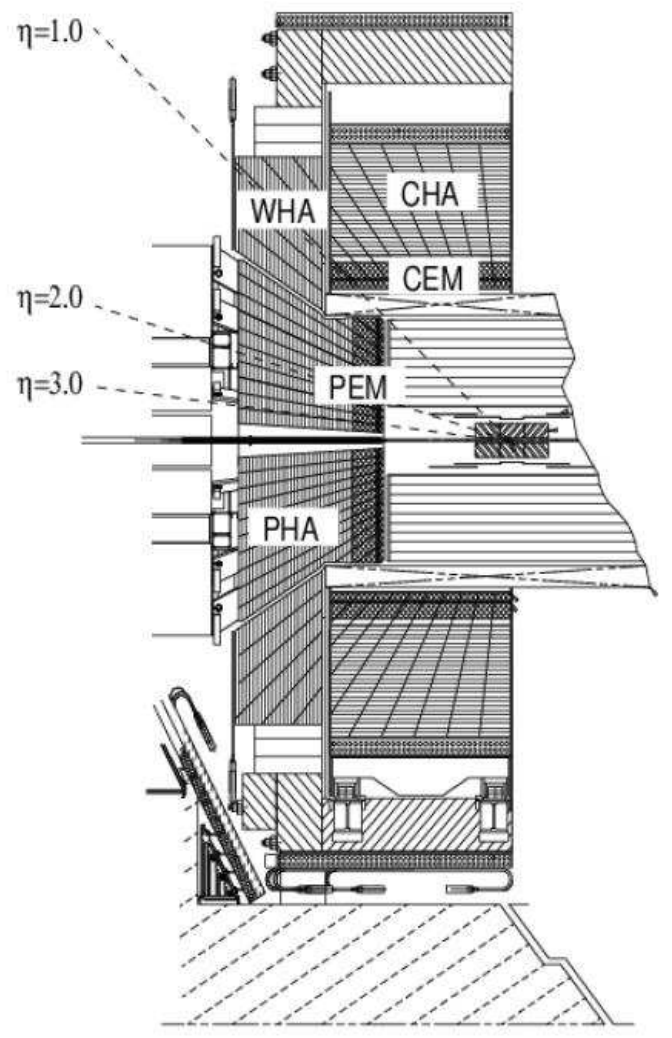

Figure 2.5: The CDF calorimeter system.

\section{Central Electromagnetic Shower Maximum Detector (CES)}

The CES is a proportional chamber with wire and strip readout which improves the position resolution of the calorimeter clusters. The CES is placed at a position at which the shower for electrons and photons has the maximum number of particles, called the shower maximum. Its position corresponds to a depth of $6 X_{0}$ of the EM calorimeter. The position resolution is $0.2 \mathrm{~cm}$ at $50 \mathrm{GeV}$.

\section{Plug Electromagnetic Calorimeter (PEM)}

The PEM is segmented into 12 towers in $\eta$ and 24 (48) towers in $\phi$ for the inner (outer) groups. It is a lead-scintillator sampling calorimeter having a total thickness is $21 X_{0}$. The energy resolution of the PEM is:

$$
\frac{\sigma_{E}}{E}=\frac{14.4 \%}{\sqrt{E(\mathrm{GeV})}} \oplus 0.7 \%
$$

\section{Plug Electromagnetic Shower Maximum Detector (PES)}

The PES [42] measures the shower maximum position similar to the CES. It is located at $6 X_{0}$ depth and is made of two layers of $5 \mathrm{~mm}$ wide scintillator strips, with each layer 


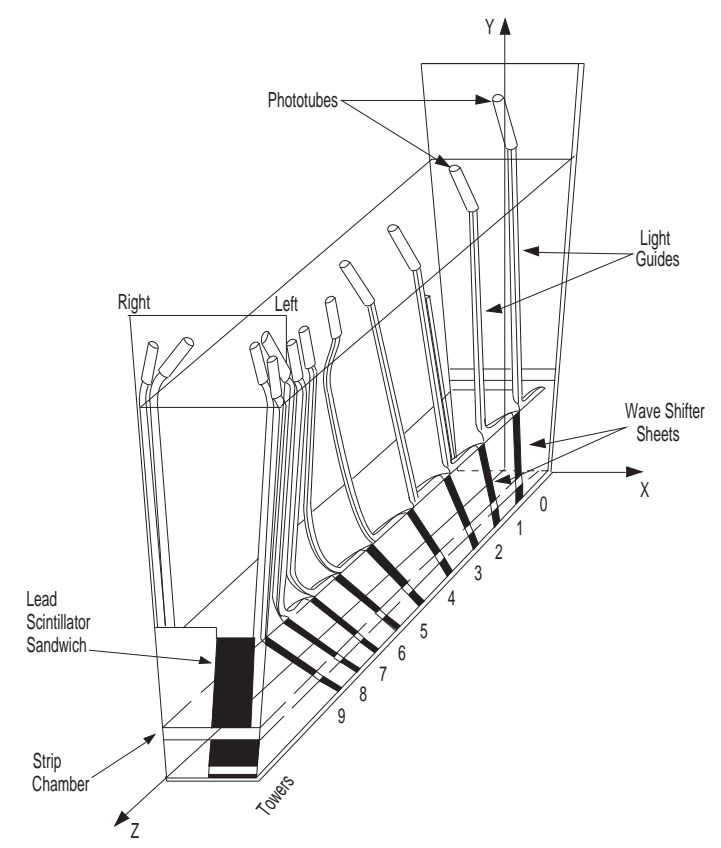

Figure 2.6: The single CEM wedge.

having a $45^{\circ}$ crossing angle relative to the other.

\section{Central Hadronic Calorimeter (CHA)}

The CHA, a iron-scintillator sampling calorimeters, is segmented into 24 towers in $\phi$ and 8 towers in $\eta$ [43]. It is located directly outside of the CEM with 32 layers per tower, which corresponds to 4.7 interaction lengths $\left(\lambda_{I}\right)$ thick. The energy resolution of the CHA is:

$$
\frac{\sigma_{E}}{E}=\frac{50 \%}{\sqrt{E(\mathrm{GeV})}} \oplus 3 \%
$$

\section{Wall Hadronic Calorimeter (WHA)}

The WHA extends the CHA coverage to fill the gap between the central and plug regions [43]. It is made of 15 layers of iron $(5.0 \mathrm{~cm})$ and scintillator $(1.0 \mathrm{~cm})$. The energy resolution of the WHA is:

$$
\frac{\sigma_{E}}{E}=\frac{75 \%}{\sqrt{E(\mathrm{GeV})}} \oplus 4 \%
$$

\section{Plug Hadronic Calorimeter (PHA)}

The PHA is made of 23 layers of alternating iron and scintillator. The energy resolution of the PHA is:

$$
\frac{\sigma_{E}}{E}=\frac{80 \%}{\sqrt{E(\mathrm{GeV})}} \oplus 5 \%
$$




\subsubsection{Muon Detectors}

The energy of high- $p_{T}$ muons are not measurable with the calorimeter, since they pass through the detector materials by depositing only minimum ionizing energy. The CDF muon detectors consist of four systems and are located outside of the calorimeters. The muon system coverage is shown in Figure 2.7. A cross section view of a muon chamber is shown in Figure 2.8. When a muon passes through the muon system, the drift time in each layer is registered. We define a muon "stub" which requires a hit in three of the four layers of drift chambers.

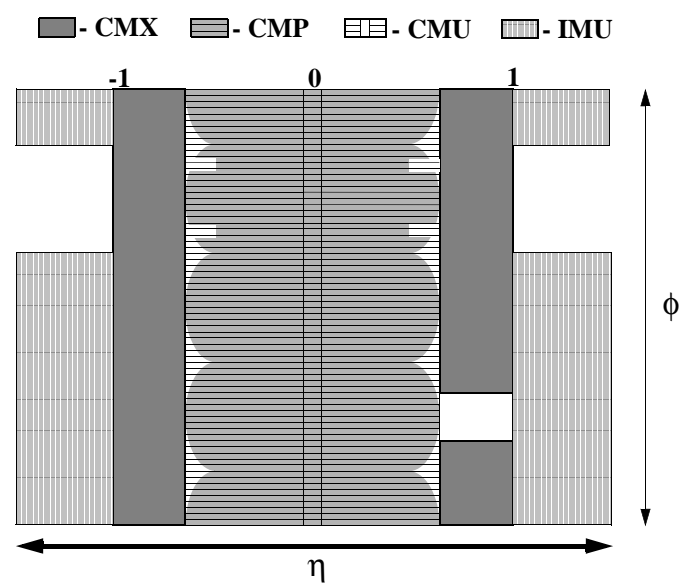

Figure 2.7: CDF muon coverage for the CMU, CMP, CMX, and BMU detectors. The BMU is referred as the IMU.

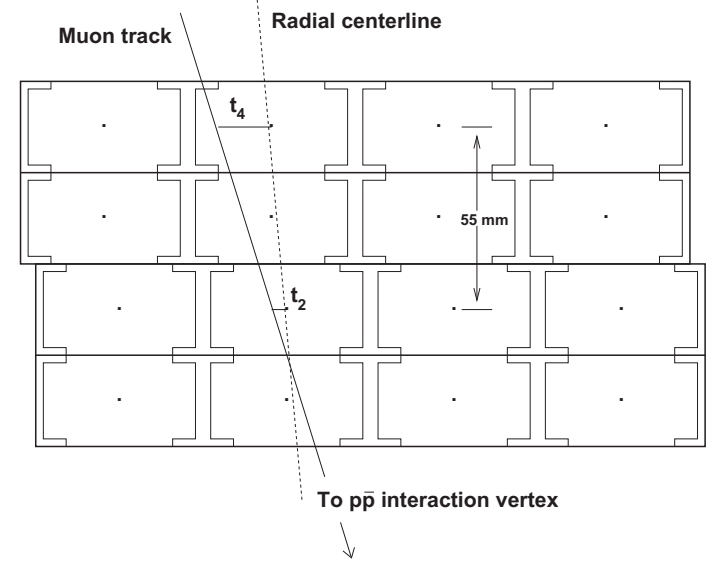

Figure 2.8: A cross section view of a CMU muon chamber. 


\section{Central Muon Detector (CMU)}

The CMU [44] consists of four layers of planar drift chambers located outside of the CHA. It covers the central region of $|\eta|<0.6$ and can detect muons with $p_{T}>1.4 \mathrm{GeV} / c$.

\section{Central Muon Upgrade (CMP)}

The CMP consists of four layers of planar drift chambers located outermost of the CDF for walls or behind the magnet return yokes. The CMP covers $|\eta|<0.6$ and can detect muons with $p_{T}>2.0 \mathrm{GeV} / c$.

\section{Central Muon Extension (CMX)}

The CMX consists of four to eight layers of drift chambers depending on the polar angle. It provides muon detection in the region $0.6<|\eta|<1.0$ and can detect muons with $p_{T}>1.4 \mathrm{GeV} / c$. The scintillator tiles (CSX) is also placed on the inside and outside of the CMX, used for improved triggering.

\section{Barrel Muon Detector (BMU)}

The BMU extends the muon detector coverage to $1.0<|\eta|<1.5$. Drift chambers and scintillators are attached surrounding the forward toroid magnets.

\subsubsection{Luminosity Monitor}

\section{Luminosity}

The collision rate of protons and antiprotons is quantified by the instantaneous luminosity. The luminosity is calculated with the following formula:

$$
\mathcal{L}=\frac{f_{r} N_{B} N_{p} N_{\bar{p}}}{2 \pi\left(\sigma_{p}^{2}+\sigma_{\bar{p}}^{2}\right)} F\left(\frac{\sigma_{l}}{\beta^{*}}\right)
$$

where $f_{r}$ is the revolution frequency, $N_{B}$ is the number of bunches, $N_{p}$ and $N_{\bar{p}}$ are the number of protons or antiprotons per bunch, $\sigma_{p}$ and $\sigma_{\bar{p}}$ are the beam sizes at the interaction point, $F$ is a form factor which corrects for the bunch shape and depends on the ratio of the bunch length $\sigma_{l}$ to the beta function $\beta^{*}$ at the interaction point. $\beta^{*}$ is a measure of the beam spreads, which are given by $\sqrt{\beta^{*} \epsilon}$ with $\epsilon$ being the beam emittance.

There is a continuous effort to maximize the peak luminosity which directly results in increasing the amount of data delivered by the Tevatron. The amount of data collected through Run II is expressed by the integrated luminosity $\left(\int \mathcal{L} \mathrm{dt}\right)$ which is measured in units of $b^{-1}$, where $1 \mathrm{~b}^{-1}$ is $10^{24} \mathrm{~cm}^{-2}$. Figure 2.9 shows the integrated luminosity delivered by the Tevatron and recorded by the CDF. 


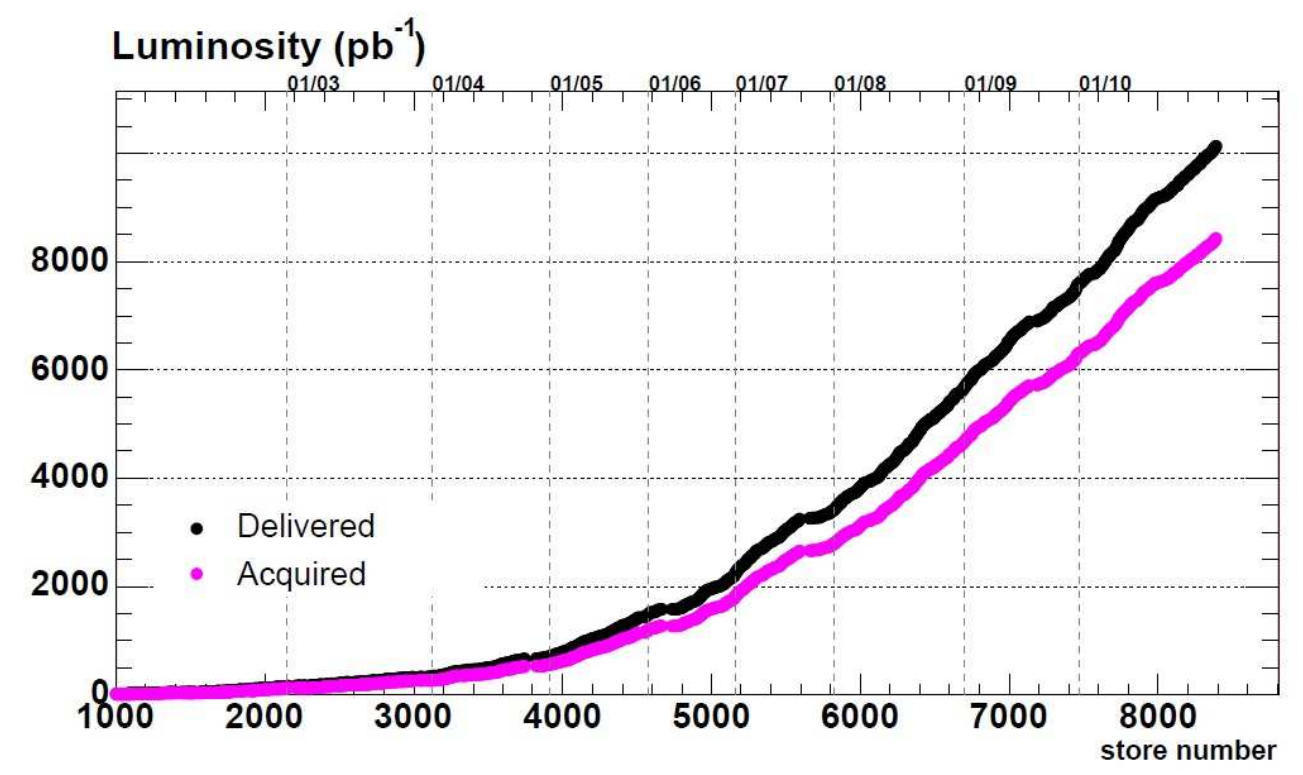

Figure 2.9: Total integrated luminosity delivered by the Tevatron and recorded by the CDF.

\section{Cherenkov Luminosity Counters (CLC)}

The beam luminosity is measured by using the CLC detector [45] located in the forward region $(3.7<|\eta|<4.7)$ of the CDF detector on both sides. The CLC consists of long conical gaseous Cherenkov counters that monitor the average rate of inelastic $p \bar{p}$ collisions per bunch crossing $\left(R_{p \bar{p}}\right)$. The instantaneous luminosity $\left(\mathcal{L}_{\text {inst }}\right)$ is calculated from the next expression:

$$
\mu \cdot f_{B C}=\sigma_{i} \cdot \mathcal{L}_{\text {inst }} \cdot \epsilon
$$

where $\mu$ is the number of interactions per bunch crossing counted by the CLC detector, $f_{B C}$ is the bunch crossing frequency $(2.5 \mathrm{MHz}$ for $36 \times 36$ bunch operations), $\epsilon$ is the CLC acceptance for a single $p \bar{p}$ interaction, and $\sigma_{i}$ is the inelastic $p \bar{p}$ cross section at the Tevatron $(60.7 \pm 2.4 \mathrm{mb})$.

\subsubsection{Data Acquisition System}

The bunch crossing rate of the Tevatron is $2.5 \mathrm{MHz}$ for $36 \times 36$ bunch operations which corresponds to $396 \mathrm{~ns}$ separation. The actual interaction rate is bit lower, about $1.7 \mathrm{MHz}$. Since this rate is too high to record every event into disk, we need to discard the most events while interesting ones must be identified. The selection of events is performed by the fast online electronics, called the trigger system. The CDF trigger system has a three level architecture (called Level 1, Level 2, and Level 3) and is designed to reduce the data rate by identifying the physically interesting events. Once an event is accepted by the Level 3 trigger, then the data are sent to the Consumer Sever/Logger (CSL) that is the final component in the CDF data acquisition system. The data flow in the CDF trigger system is shown in Figure 2.10. 


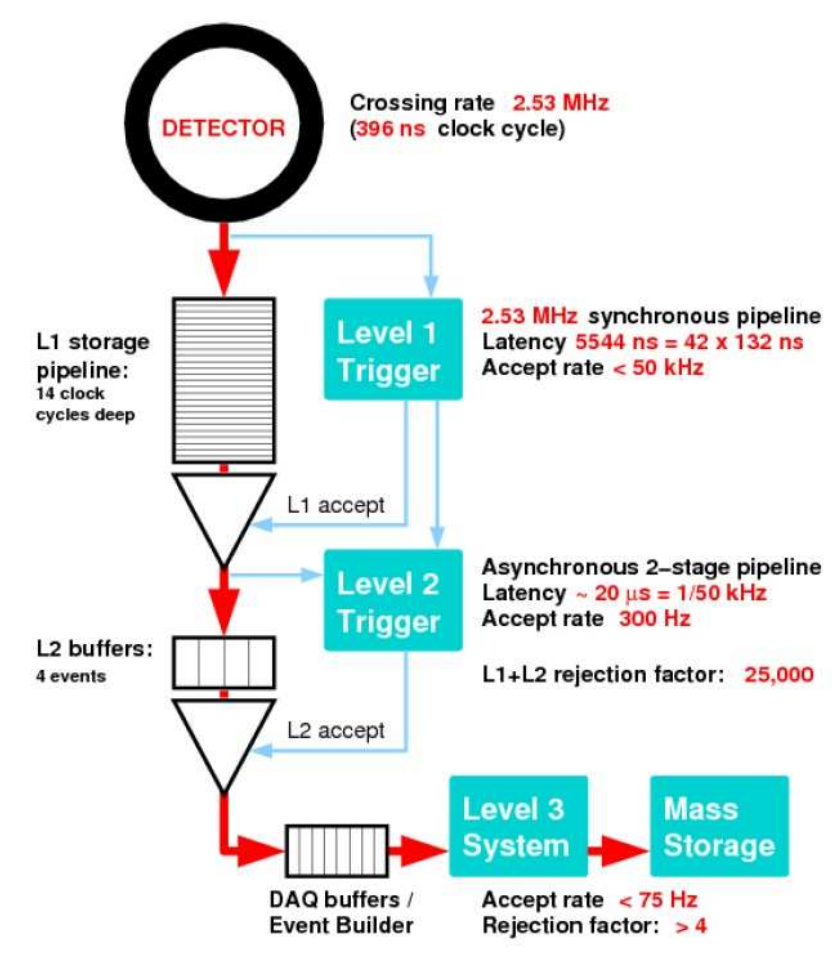

Figure 2.10: Data flow in the CDF data acquisition system.

\section{Level 1}

The Level 1 trigger (L1) discards about $97 \%$ of the events, resulting in the acceptance rate to about $50 \mathrm{kHz}$. Figure 2.11 shows the trigger path from the Level 1 to the Level 2. The L1 CAL can make its decision based on clusters of energy in the calorimeters, missing energy from the energy conservation, or the sum of calorimeter energy. A system called Extremely Fast Tracker (XFT) [46] reconstructs tracks using the COT information, and the tracks found by the XFT are used for the L1 trigger decision. The CMU can also provide L1 trigger for muon candidates.

\section{Level 2}

The Level 2 trigger (L2) reduces the event rate to about $300 \mathrm{~Hz}$. The L2 system consists of several asynchronous subsystems which provide input to programmable L2 processors. The L2 decisions are made based on the following:

- L2 cluster finder (L2CAL): The L2CAL combines adjacent calorimeter towers over $1 \mathrm{GeV}$ threshold starting from a seed tower of minimum $3 \mathrm{GeV}$.

- CES information: The shower maximum detector information provides the position resolution for electron and photon showers with better than the cluster location. It is also used to match with the tracking information. 


\section{RUN II TRIGGER SYSTEM}

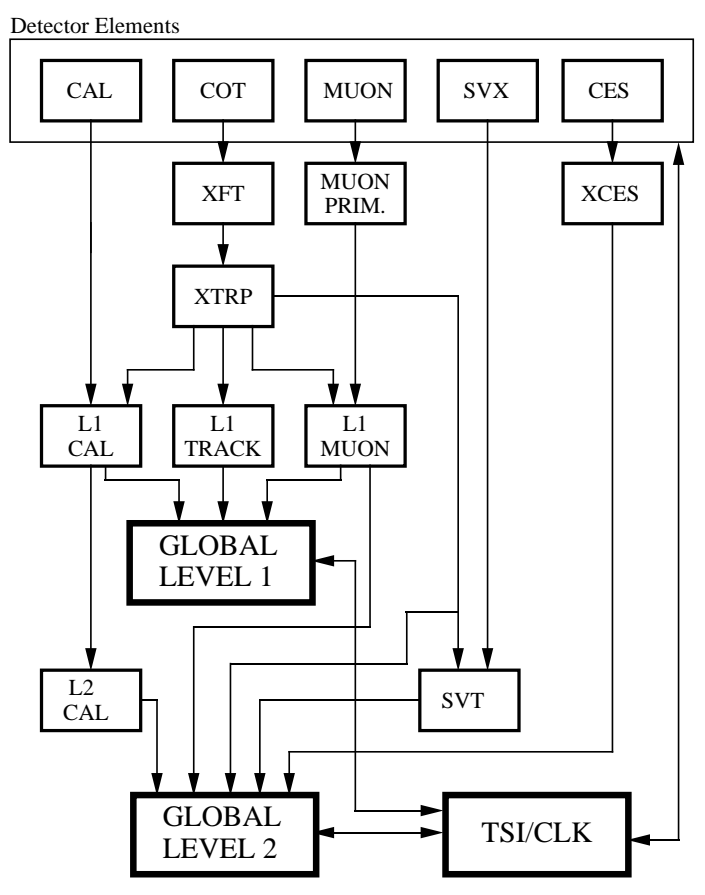

PJW 9/23/96

Figure 2.11: Block diagram of the Level 1 and Level 2 trigger path.

- Silicon Vertex Tracker (SVT) [47]: The SVXII information is combined with the L1 XFT track information by the SVT, a system that rapidly analyzes the silicon data to look for a displaced vertex.

- Muon information: The muon trigger combines information from the muon detector and from the L1 XFT track.

\section{Level 3}

The Level 3 trigger (L3) consists of a few hundred of computers. The L3 computer farm reconstructs the event in software and filter the event rate to about $75 \mathrm{~Hz}$. Events that pass L3 decision are written to disk.

\section{Consumer Server/Logger}

Once an event is accepted by the L3 trigger, it sends to the Consumer Server/Logger (CSL) system. The CSL is responsible for categorizing events by the trigger path, writing them to the disk, and sending a fraction of events to online processors for real time monitoring of data quality. 


\section{Chapter 3}

\section{Reconstruction and Event Selection}

As outlined in Chapter 1, the $t \bar{t}$ dilepton events $\left(p \bar{p} \rightarrow t \bar{t} X \rightarrow b W^{+} \bar{b} W^{-} X \rightarrow b \bar{b} l^{+} \nu l^{\prime} \bar{\nu} X\right)$ have a signature that is characterized by two high- $p_{T}$ leptons, electrons or muons, two high- $E_{T}$ jets and two neutrinos resulting in a large transverse energy imbalance, missing transverse energy, $\mathbb{E}_{T}$.

This chapter describes in detail the identification variables and criteria applied to identify final state objects in the event by the detector components. We start with the electron identification in the central and plug regions of the detector, discuss the requirements for muon candidates, describe the jet reconstruction algorithm and corrections to the jet energies, and the way we determine the energy of neutrinos escaping the detector.

\subsection{Electron Identification}

Electrons resulting from the dilepton channel of the $t \bar{t}$ decay are highly energetic. They can be identified by a high- $p_{T}$ track in the drift chamber and large energy depositions in electromagnetic calorimeters. At energies of tens of $\mathrm{GeV}$ the dominant energy loss for electrons is bremsstrahlung. When electrons traverse the lead absorbers in the electromagnetic calorimeter, they interact with the nuclei of the material and emit photons which produce electron-positron pairs $e^{+} e^{-}$. The secondary particles are also very energetic and lead to production of an electromagnetic cascade, called a shower. The shape and position of the electromagnetic shower is measured by shower maximum detectors, and the shower characteristics are used in electron identification. A hadronic shower is longer and much broader. Electrons deposit most of their energy in a single electromagnetic calorimeter tower, while a hadronic shower continues into the hadronic section of the calorimeter and into the adjacent calorimeter towers. In addition to certain shower properties, for electrons the momentum of the track pointing to the shower should match the energy in the calorimeter.

\subsubsection{Central Electrons}

Central electron candidates traverse the central part of the detector, $|\eta|<1.1$, leaving the track in the COT and depositing most of their energies in the CEM calorimeter. The following variables and criteria are used to identify high- $p_{T}$ electrons: 
- $E_{T}=E \sin \theta>20 \mathrm{GeV}$

The transverse electromagnetic energy $E_{T}$ deposited by the electron candidate in the CEM cluster. It is given by the total electromagnetic energy of the electron cluster $E$ multiplied by $\sin \theta$ of the COT track pointing to the seed tower of the cluster. An electron cluster is formed of a seed EM tower, where are added to the seed tower until the maximum cluster size is reached. The cluster at maximum has two towers in pseudorapidity and one tower azimuth. The energy $E$ is corrected for differences due to non-linearities and time-dependent changes.

- $p_{T}>10 \mathrm{GeV}$

The transverse momentum by the COT track measured by its curvature in the magnetic field. Raw COT resolution is substantially improved by constraining the track to originate from the beam line. The beam constrained tracking introduces a $p_{T}$ curvature bias in data, which is removed by correcting the signed curvature $Q / p_{T}$, where $Q$ is the charge of the track:

$$
\frac{Q}{p_{T}^{\text {corr }}}=\frac{Q}{p_{T}}-0.00037-0.0011 \times \sin (\phi+0.28)
$$

Later in the text we refer to the $p_{T}$ of the COT track when beam constrained, $p_{T}^{\text {corr }}$. This correction is applied only to data and not to simulation of events.

- $E_{\text {had }} / E_{\text {em }}<0.055+0.00045 \times E_{\text {total }}$

The ratio of the hadronic calorimeter (CHA) energy of the cluster $E_{\text {had }}$ to its electromagnetic energy $E_{\text {em }}(\mathrm{CEM})$. The value $E_{\text {had }} / E_{\text {em }}$ is scaled by a factor of 0.00045 multiplied by the total energy of the cluster $E_{t o t a l}$ to compensate for inefficiency of the cut at very high energies, as higher energy electrons have more leakage into the hadronic calorimeter.

- $E / P<2$ is applied for electrons with $E_{T}<199 \mathrm{GeV}$ or $p_{T}<50 \mathrm{GeV}$.

The ratio of the electromagnetic calorimeter energy $E$ to the COT track momentum $P$. During the passage through the material inside the COT inner radius the electron might radiate a photon ("external bremsstrahlung"). The photon is collinear with the electron and it generally deposits its energy in the same calorimeter tower, thus not much affecting the value of $E_{T}$, however the momentum $p_{T}$ measured in the COT after bremsstrahlung will be smaller. This causes a long tail in the $E / P$ distribution above 1.0 .

- $L_{\mathrm{shr}}<0.2$,

The lateral shower profile $L_{s h r}$ is a measure of how well the lateral shower development matches that expected from the electromagnetic shower. This variable compares the energies of CEM calorimeter towers adjacent to the seed tower of the EM cluster with energies expected from the test beam electrons. It is defined as a sum over towers: 


$$
L_{s h r}=0.14 \sum_{i} \frac{E_{i}^{\text {measured }}-E_{i}^{\text {expected }}}{\sqrt{(0.14 \sqrt{E})^{2}+\sigma_{E_{i}^{\text {expected }}}^{2}}}
$$

where measured $E_{i}$ is a measured energy in the CEM tower $\mathrm{i}$; $E^{\text {expected }} i$ is an energy deposit in the $i$-th tower expected from the test beam electrons and $E$ is the energy of the EM cluster. $0.14 \sqrt{E}$ represents the error on the energy measurement and $\sigma_{E_{i}^{e x p e c t e d}}^{2}$ is the uncertainty on the energy estimate. For a typical EM cluster $L_{s h r}$ is a two-tower sum. Any extra particles accompanying the one responsible for the main EM shower will tend to add to the energy in adjacent tower and make $L_{s h r}$ a large positive number.

- $-3.0 \mathrm{~cm}<\mathrm{Q} \Delta \mathrm{x}<1.5 \mathrm{~cm} ;|\Delta \mathrm{z}|<3 \mathrm{~cm}$

The distance $\Delta x /(|\Delta z|)$ in the $r-\phi /(r-z)$ plane between the COT track extrapolated to the CES and the best matching CES cluster. The cut on $\Delta x$ has been multiplied by the charge $\mathrm{Q}$ of the electron and it is asymmetric in $r-\phi$ to account for energy of the photon emitted in bremsstrahlung radiation. This requirement on a tight match between the track and shower position removes a large number of fake electron due to the coincidence of charged and neutral hadrons in the same tower, mainly due to $\pi^{0}$ and $\pi^{ \pm}$results in a reconstructed track that matches the electromagnetic cluster.

- $\chi_{\text {strip }}^{2}<10$

The $\chi^{2}$ comparison of the CES shower profile in the $r-z$ view with the shower profile extracted form test beam electrons. The $\chi^{2}$-fit is performed on the distribution of energy deposited on each of the 11 strips in the CES shower.

- $\left|z_{\text {vertex }}\right|<60 \mathrm{~cm}$

The interaction position in $z, z_{\text {vertex }}$, is taken from $z_{0}$, the $z$ intersection of the track with the beam axis in the $r-z$ plane. The longitudinal spread of the event vertex about the nominal interaction pint $z=0$ is a Gaussian with $\sigma=26 \mathrm{~cm}$. The vertex position is required to be within $2 \sigma$.

- Track quality cuts

To insure that the track associated with electron is well reconstructed, it must pass through two axial and three stereo superlayers (SL) of COT with at least 7 hits out of 12 in each SL.

- Fiduciality

This variable insures that the electron is reconstructed in a region of the detector that is well instrumented. The electron position in the CEM is determined by the CES shower position and it must satisfy the following requirement. 
- the electron must lie with $21 \mathrm{~cm}$ of the tower center in the $r-\phi$ view, so that the shower is fully contained in the active region, this corresponds to the cut $\left|x_{C E S}\right|<21 \mathrm{~cm}$, where the $x_{C E S}$ is the local coordinate of the calorimeter tower.

- the region $\left|x_{C E S}\right|<9 \mathrm{~cm}$, where the two halves of the central calorimeter meet is excluded, as well as the region $\left|z_{C E S}\right|>230 \mathrm{~cm}$, which corresponds to the outer half of the CEM tower (tower9), as it is more subjected to the leakage into the hadronic part of the calorimeter.

- the region immediately close to the point of penetration of the cryogenic connections to the solenoidal magnet, the chimney, is un-instrumented and therefore excluded. It corresponds to $0.77<\eta<1.0,75^{\circ}<\phi<90^{\circ}$ and $\left|z_{C E S}\right|>193 \mathrm{~cm}$.

- the region $1.05<|\eta|<1.10$ is excluded due to the smaller depth of the electromagnetic calorimeter.

\section{- Not a conversion}

Photons produced either directly in the hard scattering or from hadron decays interact with the material in the detector and convert to electron-positron paris. These photon conversion can be identified by the presence of another track of the opposite sign near the electron candidate. If the two tracks exhibit small $r-\phi$ separation in the pint of conversion $|\Delta X Y|<0.2 \mathrm{~cm}$, and the difference in their polar angle $|\Delta \cot \phi|<0.04$, the electron candidate is flagged as a conversion and the whole event is rejected.

- Isolation $=E_{T}^{\text {iso }} / E_{T}^{\text {cluster }}<0.1$.

where $E_{T}^{\text {iso }}=E_{T}^{0.4}-E_{T}^{\text {cluster }}$ is the transverse energy $E_{T}^{0.4}$ in a cone of radius $\Delta R=$ $\sqrt{(\Delta \eta)^{2}+(\Delta \phi)^{2}} \leq 0.4$ around the electron cluster (hadronic + electromagnetic) excluding the electron cluster energy $E_{T}^{\text {cluster }}$. This cut in fact is not an electron identification requirement. It rejects the electrons that are not isolated from extra hadronic activity and could be the products of quark semi-leptonic decays, while electrons from $\mathrm{W}$ and $\mathrm{Z}$ decays are expected to be isolated. We therefore consider both categories of isolated and non-isolated electrons in the event selection.

The isolation is corrected for leakage energy into the neighboring $\phi$ wedge outside of the cone, which increases towards the edges of the $\phi$ wedges. The respective correction factor is determined form Monte Carlo data comparison and parametrized the form:

$$
E_{\text {leak }}=E_{T}^{\text {cluster }} \cdot P_{0} \cdot \exp P_{1} \cdot\left(\left|x_{C E S}\right|-21\right)
$$

where $P_{0}=0.0511 \pm 0.0075$ and $P_{1}=0.33 \pm 0.061$. If additional interactions occur in the same bunch crossing the energy in a cone is increased. Therefore isolation is also corrected for the number of interactions par bunch-crossing, the efficiency of the cut then becomes independent of the instantaneous luminosity. This correction factor is determined similarly to jet multiple interaction energy correction. 
The corrected isolation energy is then

$$
E_{T}^{\mathrm{corr}}=E_{T}^{\mathrm{iso}}-E_{\mathrm{leak}}-E_{\mathrm{MI}}
$$

and corrected isolation is given by

$$
I s O_{\mathrm{corr}}=E_{T}^{\mathrm{corr}} / E_{T}^{\mathrm{cluster}}
$$

Central electron ID efficiency was determined from the $Z \rightarrow e^{+} e^{-}$events were selected as dilepton events of opposite charge falling into $\mathrm{Z}$ mass window, $75 \mathrm{GeV}<\mathrm{M}_{\mathrm{ee}}<$ $105 \mathrm{GeV}$. The number of observed same-sign dielectron event's is the same $\mathrm{Z}$ mass range served as an estimate of QCD background contamination. At least one electron was required to be tight, i.e. passing all identification cut.

\subsubsection{Plug Electrons}

Electron candidates deposition their energies in the PEM calorimeter are referred to as plug electrons. We impose the following identification criteria to define plug electron candidates:

- $1.2<|\eta|<2.0$

Although plug electron can be identified up to $|\eta|<2.5$, this analysis considers only those with $|\eta|<2.0$, primarily due to large charge misidentification rate at high pseudorapidities $\eta$. This cut has a small effect on the $t \bar{t}$ acceptance, as final products of $t \bar{t}$ decay events are mostly central and the acceptance falls rapidly at large $\eta$, while backgrounds considerably increase. The low $\eta$-region $|\eta|<1.2$ is excluded due to geometrical reasons since the PES detector does not provide usable coverage there. The track information from the COT is unavailable in the forward region of the detector, as plug electrons do not traverse the active volume of the COT. Plug electron candidates identified based only on the presence of an EM cluster in the PEM calorimeter, i.e. with no track requirements, are called PEM electrons. PEM electron candidates do not provide charge sign information and are subject to a large fake rate.

To reduce the misidentification rate the track reconstruction for plug electrons is performed by utilizing the silicon layer (ISL) residing in the forward part of the detector and providing usable coverage in $1.2<|\eta|<1.8$ rage. The silicon pattern recognition code extrapolates the hits in the outer layers to the inner layers of silicon and determines the location of the primary vertex. The tracking efficiency for tracks pointing to the plug region is considerably lower than for the central part of the detector. It is improved by a special algorithm, called phoenix algorithm. the Phoenix algorithm makes use of the information of the PES position of an EM shower. It constraints the track at two endpoints, one is fixed at the interaction vertex and the other is at the PES position of an EM shower. Adoption the corresponding PEM cluster energy as the momentum of the electron, a helix of the track can be determined. This defines two possible track trajectories, one is for negative 
and the other is for positive charge. The silicon pattern recognition code further attempts to reconstruct those tracks by matching hits in the layers of silicon. If one of these tracks is reconstructed, it is appended to the event record as being associated with the respective electron candidate. If both tracks are reconstructed then the algorithm performs the $\chi^{2}$-fit of a possible electron trajectory and adopts the track best matching the activity in the silicon detector. Such a track is call a Phoenix plug electron, abbreviated as PHX.

The Phoenix algorithm establishes charge identification for plug electron candidates beyond the coverage of the forward silicon layers up to $|\eta|<2.0$.

- $E_{T}>20 \mathrm{GeV}$

Unlike the CEM clusters, the PEM clusters are limited to $2 \times 2$-two towers in pseudorapidity by two towers in azimuth.

- $E_{\text {had }} / E_{\text {em }}<0.05$

Similar to the central electrons, the ratio of the hadronic calorimeter (PHA) energy of the cluster $E_{\text {had }}$ to its electromagnetic energy $E_{\mathrm{em}}(\mathrm{PEM})$.

- $U_{5 \times 9} \geq 0.65$ and $V_{5 \times 9} \geq 0.6$ The quantities $U_{5 \times 9}$ and $V_{5 \times 9}$ are essentially isolation variables for the shower maximum detector independently applied to both the $\mathrm{U}$ and $\mathrm{V}$ layers. The PES clustering is performed by ordering PES strips in decreasing energy with the highest-energy strips used as seeds. Then a fixed-width nine-strip cluster is formed from each seed. This is done separately for $1 \mathrm{D} \mathrm{U}$ - and V-layer clusters. The quantities $U_{5 \times 9}$ and $V_{5 \times 9}$ represent the ratios of energy sum in the central 5 strips of a PES cluster to the total energy of the PES cluster (in all 9 strips).

- $\chi_{3 \times 3}^{2}<10$

This variable represents the goodness of $\chi^{2}-f i t$ measure of the energy distribution in $3 \times 3$ towers around the seed tower to energy distributions from test beam electrons.

- $\left|\triangle R_{P E S}\right|<3 \mathrm{~cm}$

The $\chi^{2}-f i t$ also determines the position for the center of the shower. $\Delta R_{P E S}=$ $\sqrt{(\Delta \eta)^{2}+(\Delta \phi)^{2}}$ is the distance between the $\left.\chi^{2}\right) 3 \times 3$ best fit position and the intersection of the centroids in the U- and V-layer PES clusters. $\Delta R$ matching requirement is also enforced between the PES coordinates and the extrapolated Phoenix track.

- $N_{\text {hits }}^{\mathrm{Si}} \geq 3$

Number of Silicon hits $N_{\text {hits }}^{\mathrm{Si}}$. This requirement is enforced to improve the quality of the silicon tracks at the cost of some efficiency.

- $\left|z_{0}\right|<60$

This cut is identical to the CEM electrons. 
- Isolation $=E_{T}^{\text {iso }} / E_{T}^{\text {cluster }}<0.1$

This variable is defined identically to the CEM electrons. The corrections to $E_{T}^{\text {iso }}$ are done differently, however, as the clustering algorithm in the plug region differs from the one in the central region. The leakage energy is parametrized depending on the distance of the tower form the center of the electron shower in $\theta$ and $\phi$.

Plug electron ID efficiency was determined from the $Z \rightarrow e^{+} e^{-}$data sample, similar to how it was done for central electrons. $Z \rightarrow e^{+} e^{-}$events were selected as central-plug dielectron events in the $\mathrm{Z}$ mass range. The central electron was required to be a good electron, i.e. pass all tight identification criteria, and the other leg was required to be an EM object fiducial to the plug region. Plug electron ID efficiency was determined as the ratio of tight-tight (central-plug) candidates over tight-loose candidates.

\subsection{Muon Identification}

Muons are minimum-ionizing particles which penetrate matter very easily. Muons resulting from the dilepton channel of the $t \bar{t}$ decay, as well as electrons, are very energetic. They are identified by the high- $p_{T}$ track in COT, very little energy deposition in the calorimeters, and matching hits in the muon chambers. A muon candidate is required to have aligned hits in both $r-\phi$ and $r-z$ planes on at least 3 separate layers. These hits form a muon stub which is then matched to the COT tracks extrapolated to the muon chambers.

Muons are categorized by the detector region through which they pass. Muons reconstructed in both the CMU and CMP chambers are called CMUP muons. Due to the gaps in the muon chambers coverage there are also muon that are reconstructed only in the CMU or the CMP muon chambers. These muons are called CMU, CMP respectively. Muons with stubs in the CMX chamber are called CMX muons. The COT tracks with no muon stubs are also considered as muon candidates. Such muons are required to have minimum energy depositions in the calorimeter. These muon candidates are called CMIO's (central minimum ionizing objects). The following criteria are applied for muon candidates:

- $p_{T}>20 \mathrm{GeV}$

The transverse momentum of the COT track. The track is beam constrained and $p_{T}$ corrections are applied identically as it is done for electrons.

- $\left|z_{0}\right|<60 \mathrm{~cm}$

As for electrons, the $z$-position of the muon track is associated with the event vertex and required to be within $60 \mathrm{~cm}$ from the geometrical center of the detector.

- $\left|d_{0}\right|<0.2 \mathrm{~cm}$ for tracks with no silicon hits.; $\left|d_{0}\right|<0.02 \mathrm{~cm}$ for tracks with silicon hits.

The impact parameter $d_{0}$ is the distance between the reconstructed muon track and the beam axis in the $r-\phi$ plane. Unlike others this selection variable is based on the default muon track, not beam-constrained, and a trigger cut is applied if the track contains silicon hits. This cut forces the muon to originate from the nominal 
interaction region and substantially reduces the cosmic muon background. It also helps to remove muons from kaons and pions that decay in flight.

- Track quality cuts

Track quality cuts are identical to electron track quality cuts. The track is required to have at least 7 COT hits on at least 2 axial and 3 stereo superlayers.

- $E_{\mathrm{em}} \leq 2+\max (0,0.0115(P-100)) \mathrm{GeV}$

The energy deposited in the electromagnetic calorimeter. High $p_{T}$ muons are not expected to deposit substantial amount of energy in the electromagnetic calorimeter. the sliding cut is introduced for muons with $P<100 \mathrm{GeV}$ to increase efficiency of the cut.

- $E_{\text {had }} \leq 6+\max (0,0.0280(P-100)) \mathrm{GeV}$

The energy deposited in the hadronic calorimeter is higher, but still quite small in comparison to strongly interacting jets.

- $E_{\text {em }}+E_{\text {had }}>0.1 \mathrm{GeV}$ for stubless muon only.

Stubless muons are required to have a non-zero energy deposition in the calorimeter to limit backgrounds from electrons escaping the detector through cracks in the calorimeter.

- $|\Delta x|_{\mathrm{CMU}} \leq 7 \mathrm{~cm}$

The distance in the $r-\phi$ plane between the extrapolated COT track and the stub segment in the relevant muon chamber. The muon candidate passing this requirement falls into CMUP or CMU category.

- $|\Delta x|_{\mathrm{CMP}} \leq 5 \mathrm{~cm}$

Muons in the CMP and CMX detector traverse more material than in the CMU and experience grater deflections due to multiple scattering. Therefore, the track-to-stub matching cut are looser. This muon candidate falls into CMUP or CMP category.

- $|\Delta x|_{\mathrm{CMX}} \leq 6 \mathrm{~cm}$

CMX muon category requirement.

- $\rho_{\mathrm{COT}}>140 \mathrm{~cm}$

The COT exit radius

$$
\rho_{\mathrm{COT}}=\frac{\operatorname{sign}(\eta) \cdot z_{\mathrm{COT}}-z_{0}}{\cot (\theta)}
$$

is based on pseudorapidity $\eta$ and $z_{0}$ of the track, where $z_{C O T}=155 \mathrm{~cm}$ is the length of the COT and $\theta$ is the polar angle.

This cut is enforced only for CMX muons to eliminate the data bias due to the XFT trigger requirement that a track must leave hits in at least four COT superlayers. 
- Isolation $=E_{T}^{\text {iso }} / p_{T}<0.1$

where $E_{T}^{\text {iso }}=E_{T}^{\text {cone }}-E_{T}^{\text {tower }}$ is the difference between energy in the cone of $\Delta R=$ 0.4 around the muon track $E_{T}^{\text {cone }}$ and the amount of energy in the tower associated with the muon track $E_{T}^{\text {tower }}$.

- not-cosmic

Cosmic ray can be identified in the CDF detector as dimuon events leaving a nearly straight track and therefore mimickintg a very energetic $\mu^{+} \mu^{-}$pair. Since cosmic rays do not originate from a ppbar collision and enter the detector at random locations, this background is reduced by a cut on the track impact parameter $d_{0}$. In addition, cosmic rays appear randomly in time and can be distinguished by substantial time delay between the hits of the two muons in the hadronic calorimeter, measured by Time to Digital Converter (TDC), and by using timing information from the Time of Flight detector (TOF). All of this information is analyzed by the software code, named Cosmic Ray Tagger, which makes a decision on flagging an event as a cosmic. Events with muon identified as cosmics are rejected.

The muon ID efficiencies are measured using $\mathrm{Z}$ boson decays $Z \rightarrow \mu \mu$ similarly to electrons. Both legs are linked to a CMUP or CMX muon stub, where one leg is required to pass tight identification criteria and is matched to Level-1 trigger information, while the second leg is chosen to be fiducial to the tested detector region (e.g. CMUP, CMX), or non-fiducial to any (for stubless CMIO muons) and examined if it passed muon ID cuts thus being independent of a trigger requirement.

\subsection{Jet Reconstruction}

At high energies, as those achieved in Tevatron, jets are the dominant feature of hadron production. They result from point-like collisions of a quark or gluon from the proton with a quark or gluon from the antiproton. A jet is formed from a scattered initiating parton, which experiences fragmentation leading to the creation of a stream of energetic colorless particles emitted spatially collimated along the original parton direction.

The jets are observed as cluster of energy located in adjacent detector towers. Typically a jet contains neutral or charged pions to a lesser extent of kaons, and about 10 $\%$ of light baryons such as protons and neutrons. Pions mostly deposit their energies in electromagnetic calorimeter, while kaon and baryons leave most of their energies in the hadronic section of the calorimeter. The energy of the initial parton can be approximated by summing the tower energies within a cone of specified size. This procedure is called jetclustering. The cone size is chosen to encompass most of the jet energy without allowing a significant contribution from other event activity. It is defined in $\eta-\phi$ space by its radius, $R=\sqrt{\Delta \eta^{2}+\Delta \phi^{2}}$ and is centered at the largest calorimeter energy tower serving as a seed tower of the jet cluster. This analysis is using a cone size of $\Delta R=0.4$.

After the jet cluster is thus formed, the $E_{T}$ weighted centroid of the cluster is determined, as follows 


$$
\begin{aligned}
\eta_{\text {centroid }} & =\frac{\sum_{i=1}^{N} E_{T}^{i} \eta^{i}}{\sum_{i=1}^{N} E_{T}^{i}} \\
\phi_{\text {centroid }} & =\frac{\sum_{i=1}^{N} E_{T}^{i} \phi^{i}}{\sum_{i=1}^{N} E_{T}^{i}}
\end{aligned}
$$

where the sums are carried out over all calorimeter towers in the cluster. It defined the centroid tower and a new cone drawn around this position. This process is iterated until the cluster remains unchanged in two consecutive paths. In some cases two clusters can overlap and then they are either merged into one, if the sum of the energies in shared towers exceed $75 \%$ of the energy of the smaller cluster, or left intact.

The jet four-momentum $\left(E^{\mathrm{raw}}, p_{x}^{\mathrm{raw}}, p_{y}^{\mathrm{raw}}, p_{z}^{\mathrm{raw}}\right)$ is then determined by the following sums over the cluster towers:

$$
\begin{aligned}
& E^{\mathrm{raw}}=\sum_{i=0}^{N} E_{i} \\
& p_{x}^{\mathrm{raw}}=\sum_{i=0}^{N} E_{i} \sin \theta_{i} \cos \phi_{i} \\
& p_{y}^{\mathrm{raw}}=\sum_{i=0}^{N} E_{i} \sin \theta_{i} \sin \phi_{i} \\
& p_{z}^{\mathrm{raw}}=\sum_{i=0}^{N} E_{i} \cos \phi_{i}
\end{aligned}
$$

These quantities are referred to as raw, since they are affected by mismeasurements for a variety of reasons due to both to physics and to detector effects and are different from the true energies of the partons which initiated jets. Therefore proper corrections need to be applied to reconstruct true momentums of partons.

\subsubsection{Jet Energy Corrections}

The measured four-vector of jets generally differs from the energies of the initial partons. This is the result from both instrumental and physical effects such as low energy non-linearities, $\eta$ crack energy losses, underlying events, and clustering. Some of the corrections are decided by the measurable quantities independent of the theory, while some of them rely on the theory prediction. Thus the row jet energies measured in the calorimeter must be corrected for detector effects at first before they can be compared to physics predictions/models. The correction strategy is the followings:

\section{- Relative Corrections (Level1 Corrections)}

The first step in jet energy corrections is to correct the jets for any variation in the response with detector $\eta$. For this correction, dijet event samples are used. Since the 
transverse energy of the two jets in a $2 \rightarrow 2$ process should be equal, the energies of jets in the plug and forward calorimeters are scaled to give the energy of an equivalent jet in the central calorimeter. One well-measured central jet $(0.2<|\eta|<$ $0.6)$ is required and a scale factor is derived from the dijet balance to the second jet. The central calorimeters CEM/CHA are the best understood calorimeters in CDF and the selected region is far away from the cracks. The gain variation depending on the time (run range) in the plug calorimeters is also taken into account. The corrections for the Monte Carlo and data are determined separately since some discrepancy between data and simulation can be seen due to a lack of the materials in the detector simulation.

- Multiple Interaction Corrections (Level4 Corrections)

The multiple interaction affects the measured jet energy when the energy from these minimum bias events falls into the jet clustering cone. The transverse energy in a random cone is measured in minimum bias data and parameterized as a function of the number of vertices in the event. This transverse energy is subtracted from each jet to account for multiple interaction in the same bunch crossing as a function of the number of vertices in the event. This correction factor is a linear function of the number of reconstructed vertices in the event. Only vertices associated with at least 2 COT tracks in minimum bias events are used to decide this correction factor.

\section{- Absolute Corrections (Level5 Corrections)}

The jet energy measured by the calorimeters must be corrected for any non-linearity and energy loss in the un-instrumented regions of each calorimeter. The absolute jet corrections account for the response to particle-level energy in the central calorimeter. This correction depends on the jet fragmentation properties. The calibration point is derived using a $50 \mathrm{GeV}$ pion from test beam data. For the non-linearity response, the tuned Monte Carlo events are used for the charged and neutral particles. After fragmentation, the events are processed with a full CDF detector simulation. Each simulated event is compared to the total $p_{T}$ of all generated particles lying in a cone centered about the measured jet axis. A quadratic spline fit is used to parameterize the mean jet response as a function of $E_{T}$ for the each cone size.

- Underlying Event Corrections (Level6 Corrections)

The underlying event contains all the soft interactions except the hard one. The underlying event energies must be subtracted from the measured jet energy when these particles fall into the clustering cone. The correction procedure is the same as the multiple interaction correction. Events with only one vertex are used to determine the underlying event correction.

- Out-of-Cone Corrections (Level7 Corrections) 
The jet clustering may not include all the energy from the initiating parton. Some of the partons generated during fragmentation may fall outside the cone chosen for clustering algorithm. Out-of-cone corrections are applied in order to correct the particle-level jet energy to the parton energy (as much as theoretically allowed). These corrections are completely independent of detector/calorimeter performance and depend on the parton fragmentation functions. The correction factor is parameterized as a function of jet $p_{T}$. Jet tends to become narrower at large energies, and the fractional energy deposited outside the cone decreases.

Thus, the jet energy is corrected by

$$
p_{T}(R)=\left[p_{T}^{\mathrm{raw}}(R) \times f_{\mathrm{rel}}-\mathrm{UEM}(R)\right] \times f_{\mathrm{abs}}(R)-\mathrm{UE}(R)+\mathrm{OC}(R),
$$

where $R$ denotes the clustering cone size, $p_{T}$ and $p_{T}^{\text {raw }}$ are the corrected and row transverse momenta of jet, $f_{\text {rel }}$ is the relative jet energy correction, $\operatorname{UEM}(R)$ is the multiple interactions correction, $f_{\text {abs }}(R)$ is the absolute jet energy correction, $\operatorname{UE}(R)$ is the underlying event correction, and $\mathrm{OC}(R)$ is the out-of-cone correction.

\subsubsection{Jet Energy Scale Uncertainties}

The differences between the data and CDF simulations of the jet responses are treated as the systematic uncertainties of the jet energy scale (JES) [48]. The uncertainties are estimated for each type of jet energy corrections. The uncertainties due to the absolute scale and the jet shape (out-of-cone) are dominant. The main systematic uncertainties on the absolute scale are obtained by propagating the uncertainties on the single particle response $(E / p)$ and the fragmentation. Smaller contributions are from the calorimeter response close to tower boundaries in azimuth, and from the stability of the calorimeter calibration with time. The uncertainties from the jet shape are estimated by measuring the energy flow between cones of size 0.4 and 1.3 in both data and MC simulations. The total JES uncertainties in the central region are shown in Figure 3.1.

\subsection{Missing Energy}

Neutrinos interact only through weak interactions and therefore cannot be directly detected as they traverse the detector material. Production of neutrinos in an event can be spotted by the existence of the large imbalance in the calorimeter energy. The longitudinal component of the colliding partons is not known, but the transverse component is subject to conservation, and the sum of the transverse components of the neutrino momenta can be measured. This quantity is called missing transverse energy $\overrightarrow{\mathbb{E}_{T}}$. The missing trans-

verse energy is two-component vector $\left(E_{T_{x}} E_{T_{y}}\right)$. The raw value of $\overrightarrow{\mathbb{E}_{T}}$ is defined by the negative vector sum of the transverse energy of all calorimeter towers:

$$
\vec{E}_{T}^{\mathrm{raw}}=-\sum_{\text {tower }}\left(E_{i} \sin \theta_{i}\right) \vec{n}_{i}
$$




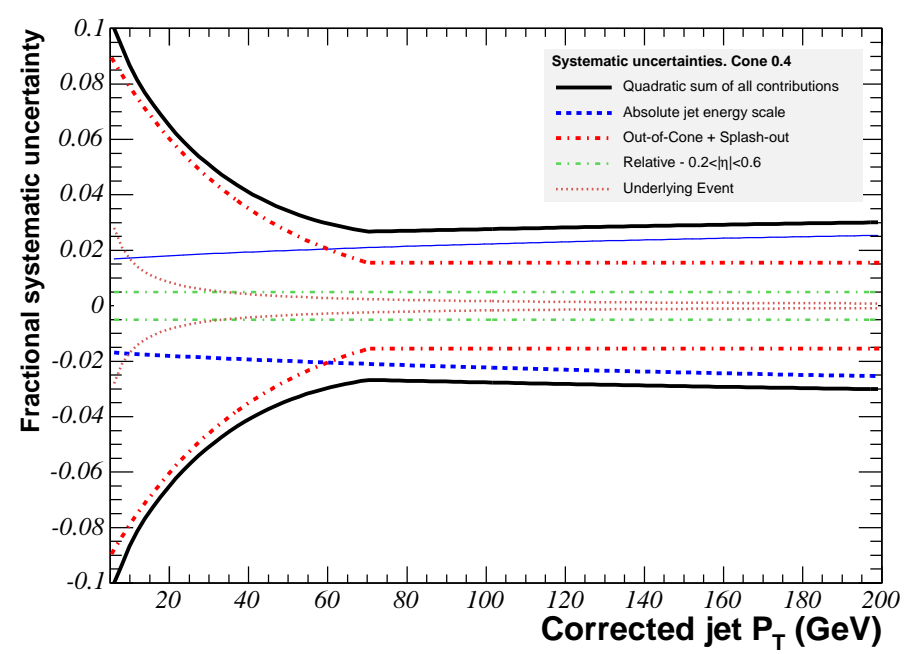

Figure 3.1: The total uncertainties of JES as a function of corrected jet $p_{T}$ in the central calorimeter $(0.2<|\eta|<0.6)$.

where $E_{i}$ is the energy of the $i$-th tower, $\vec{n}_{i}$ is a transverse unit vector pointing to the center of the tower and $\theta_{i}$ is the polar angle of the line pointing from $z_{0}, z$-coordinate of the event vertex, to the $i$-th tower. This sum extends to $\left|\eta_{\text {detector }}\right|<3.6$.

The value of $\vec{E}_{T}^{\text {raw }}$ should be further corrected for escaping muons and jet energy mismeasurements. Muons do not deposit substantial energy in the calorimeter, but may carry out significant amount of the energy. The sum of transverse momenta of escaping muons $\sum \vec{P}_{T, \mu}$ measured in the COT has to be added to the $\vec{E}_{T}^{\text {raw }}$ with a negative sign and the energy deposited by muons in the calorimeters $\sum \vec{E}_{T, \mu}$ has to be subtracted from that sum, as it has been already counted in the $\vec{E}_{T}^{\text {raw }}$.

Only raw values of jet energies contribute to the $\vec{H}_{T}^{\text {raw }}$ and these values have to be replaced in the sum by the corrected ones. The corrected value of $\vec{E}_{T}^{\text {corr }}$ is therefore given by the following relation:

$$
\vec{H}_{T}^{\text {corr }}=\vec{H}_{T}^{\text {corr }}-\left(\sum_{\text {muons }} \vec{P}_{T, \mu}-\sum_{\text {muons }} \vec{E}_{T, \mu}\right)-\left(\sum_{\text {jet }} \vec{E}_{T, j e t}^{\text {corr }}-\sum_{\text {jet }} \vec{E}_{T, \text { jet }}^{\mathrm{raw}}\right)
$$

Uncertainties in $\vec{E}_{T}^{\text {corr }}$ are dominated by uncertainties in jet energies. Mismeasurements of $\mathbb{E}_{T}$ result from jets traversing through poorly instrumented re gions of detectors, e.g. cracks, dead zones, and beam halo effects. They may also result from cosmic rays, muon misidentification and mismeasurements in muon track momenta.

The resolution of the $\mathbb{E}_{T}$ generally depends on the response of the calorimeter to the total energy deposited in the event. It is parameterized in terms of the total scalar transverse energy $\sum \mathbb{E}_{T}$, which is defined as 


$$
\sum \mathbb{E}_{T}=\sum_{\text {towers }} E_{i} \sin \theta_{i}
$$

The $\mathbb{E}_{T}$ resolution in the data is measured with minimum bias events, dominated by inelastic $p \bar{p}$ collisions. In minimum bias events the $x$ and $y$ components of $\overrightarrow{E_{T}}$ are distributed as Gaussian around zero with $\sigma_{x}=\sigma_{y}=\sigma$ :

$$
\frac{d N}{d E_{T_{x}, y}} \simeq \exp \left(-\frac{E_{T_{x}, y}^{2}}{2 \sigma^{2}}\right)
$$

The $\mathbb{E}_{T}$ resolution $\Delta=\sqrt{\left\langle E_{T}^{2}\right\rangle}$ is then given by $\Delta=\sqrt{2} \sigma_{x, y}$. It is expected to scale as a square root of the total transverse energy in the event, $\sum E_{T}$, is determined to be $\Delta \simeq 0.64 \sqrt{\sum E_{T}}$ from minimum bias studies. 


\section{Chapter 4}

\section{Spin Correlation Measurement}

\subsection{Outline of the Measurement}

In this thesis we measure the strength of the $t \bar{t}$ spin correlation $\kappa$ from a differential angular distribution of the two decay leptons and the two decay b-jets in the rest frames of their respective top quark and the spin quantization axis. Since the spin correlation is large in beamline basis and beamline basis is easier to determine experimentally, we chose beamline basis as a spin quantization axis. To measure $\kappa$, we use $t \bar{t}$ dilepton channel, since, as we discussed in the Section 1.7, the charged leptons from the $t \rightarrow W b \rightarrow \ell \nu_{\ell} b$ decays are the probes with the highest sensitivity to the direction of the top quark spin. From an angular distribution of two charged leptons, the information on $\kappa$ is to be extracted. In addition to the angular distribution of charged leptons, we use one of b-jets as well to increase the sensitivity to the $\kappa$ measurement. In order to obtain the flight directions of decay products in top and anti-top rest frames, we need to a full kinematical reconstruction of $t \bar{t}$ system in dilepton decay channel including two neutrinos. The method of the full kinematical reconstruction will be discussed in Section 4.4. Then we will make the templates of $t \bar{t}$ signal as a function of $\kappa$ as well as the background from each angular distribution(Section 4.5). Using the templates, we fit the angular distribution of data, and once we get measured $\kappa$, we set Feldman-Cousins confidence intervals on the true $\kappa$. The construction of Feldman-Cousisns confidence belt from pseudo-experiments will be discussed in Section 4.6.

\subsection{Top Dilepton Event Selection}

Dilepton event selection aims at reconstructing $t \bar{t}$ events with both W's from top decaying leptonically. It requires two fully identified electrons or muons with transverse energy above $20 \mathrm{GeV}, E_{T}>25 \mathrm{GeV}$ and at least two tight jets of $E_{T}>15 \mathrm{GeV}$. The first, or trigger lepton can be one of three types: CEM electron, CMUP or CMX muon. The second, or loose lepton, can be also non-isolated or an isolated PHX electron or one of the non-trigger muon types: CMU-only, CMP-only and CMIO. Details on the cuts used to identify each lepton category are contained in Section 3. Non isolated counterpart of the trigger leptons (i.e. NICEM electrons and NICMUP/NICMX muons) are allowed to 
trigger the event when they come together with a PHX electron to recover most of the acceptance lost by dropping the plug electron dataset.

The jets are corrected up to hadron level (i.e. we do not apply Underlying Events and Out-of-Cone Correction, jet is called parton level after these correction). We should mention here that for the purpose of correcting $E_{T}$ (which is calculated starting from the raw transverse energy deposited in each tower of the calorimeter), we used the jet energy scale calculated ignoring the Multiple Interaction correction in order to avoid overcorrection $E_{T}$ for the presence of energy due to extra interaction.

Extra event topology cuts are imposed to improve the purity of the selection:

- Dilepton invariant mass $>5 \mathrm{GeV}$, where Monte Calro cannot describe properly in this region.

- COT radius exit cut $>140 \mathrm{~cm}$ for the stubless CMIO muon candidate, which is a high $p_{\mathrm{T}}$ central track with a minimum energy deposition in the calorimeter but no matching stub in the muon chambers, in order to remove mismodeling events around the end of COT in the simulation.

- Cosmic and conversion removal: This cut reject the events which were marked as the events with the particles coming from cosmic or the events where the electrons are coming from the conversion of the photon to $e^{+} e^{-}$pair within detector. The cosmic removal is used to remove events where the muons are identified as an cosmic muons. The conversion removal is applied only to central electrons. The track associated with the electron is checked to all other tracks whether it is close in all three spatial dimensions to another track and that it has opposite charge. In such case, the electron is flagged as the conversion electron.

- Z-veto for $e e$ and $\mu \mu$ events. To remove events that contain leptons come from $\mathrm{Z}$ boson, if the event has same flavor lepton pair with its invariant mass in $76<M_{\ell \ell}<$ $106 \mathrm{GeV}$, the missing $E_{T}$ significance $E_{T} / \sqrt{\sum E_{T}}>4 \sqrt{\mathrm{GeV}}$ is required. This variable separates events with real $E_{T}$ due to neutrinos from events where $E_{T}$ is due to energy measurement fluctuations or energy loss in calorimeter cracks. These second category of events is expected to have a degraded $E_{T}$ resolution.

- L-cut in the $\left(E_{T}, \delta \phi_{\min }\right)$ plane to reject $Z \rightarrow \tau \tau$ events and events with mismeasured $E_{T}$ from jets pointing to cracks in the calorimeter: this cut requires the event $E_{T}$ to be above $50 \mathrm{GeV}$ is there is any lepton or jet inside $20^{\circ}$ of the $\mathbb{E}_{T}$ direction. In events with mismeasured jets, the fake $\mathbb{E}_{T}$ is pointing close to the direction of the jet. Moreover, in $Z \rightarrow \tau \tau$ events the $\mathbb{E}_{T}$ vector often points close to the direction of one of the leptons. This cut remove these backgrounds.

- Scalar sum of $E_{T}^{\prime} s$ of leptons, jets, and missing energy: $H_{T}>200 \mathrm{GeV}$. Because of such heavy top quark, there is higher activity in transverse plane in $t \bar{t}$ events comparing to background events. Therefore, the variable which sums (scalarly) transverse energies of all particles in the event has higher value for $t \bar{t}$ events than in background events. Such variable we call $H_{T}$ and in our case it is the sum of $\mathbb{E}_{T}$ plus leptons transverse energies plus the $E_{T}$ of all tight jets in the event. 
- Opposite charge for the two leptons. This cut requires that the charged leptons have the charges with the opposite sign. In $t \bar{t}$ events the charged leptons have indeed the opposite sign of charges because they are coming from opposite charged top quarks (top quark vs. antitop quark). However, some of the backgrounds (Fake events) do not necessary have opposite sign leptons, so such events are suppressed by this cut.

- $\left|\delta Z_{\ell}\right|<4 \mathrm{~cm}$ is made to make sure that the two leptons are from the same interaction, where $\left|\delta Z_{\ell}\right|$ is the absolute value of the $\mathrm{Z}$ position difference of the two leptons. This cut remove events from multiple interaction.

The data we use in this analysis were collected during the period March 2002 - June 2009. The total integrated luminosity for this period is $5.1 \mathrm{fb}^{-1}$. Finally the number of observed events survived after this selection criteria are 334 events.

\subsection{Background Composition}

We consider four different sources of standard model process that can mimic the signature of dilepton plus $E_{T}$ plus two or more jets signature: diboson events ( $W W, W Z, Z Z$ or $W \gamma)$, Drell-Yan production of tau leptons $(\mathrm{Z} \rightarrow \tau \tau)$, Drell-Yan production of electrons or muons with additional $\mathbb{E}_{T}$ (if the event is an actual Drell-Yan event, there is no $\mathbb{E}_{T}$ so we refer to this as fake $\left.\mathbb{E}_{T}\right)(\mathrm{DY} \rightarrow e e / \mu \mu)$, and QCD production of $W$ boson with multiple jets in which one jet is misidentified as a lepton ( $W+$ jets fakes).

\section{- Diboson}

The diboson processes, $W W, W Z, Z Z$ and $W \gamma$, can mimic the signature of the $t \bar{t}$ signal via different mechanisms, with real leptons and $E_{T}$ from $W$ and $Z$ decays and jets produced by boson hadronic decays or initial state radiation for $W W$ events, the two leptons and the $E_{T}$ are produced when both $W$ 's decay semi-leptonically but the jets require some hadronic radiation external to the diboson system. For $W Z$ and $Z Z$ events, the two leptons come from the $Z$ boson while the other $W$ or $Z$ boson provides the jets vis their hadronic decays. As these decays do not contain any neutrino, some mechanism to produce fake $\mathbb{E}_{T}$ is required. Finally for $W \gamma$ events, one lepton plus $E_{T}$ is generated from the semi-leptonic $W$ decay while the second lepton is produced from an asymmetric $\gamma$ conversion in which one of the two electrons has little energy and is caught spiralling inside the central drift chamber.

- Drell-Yan to $e e / \mu \mu$

$Z / \gamma^{*} \rightarrow e e / \mu \mu$ process can mimic the signature of the $t \bar{t}$ signal when there are at least 2 jets from initial state radiation. More ever, this process does not have real physics $E_{T}$, it can have $E_{T}$ just due to mis-measurement of jets and/or leptons energies.

- $\mathrm{Z} \rightarrow \tau \tau$ 
$Z / \gamma \rightarrow \tau^{+} \tau^{-}$events can fake the dilepton $+E_{T}$ plus 2 or more jets signature when both $\tau$ 's decay semi-leptonically to $\ell \nu_{\ell} \bar{\nu}_{\tau} \ell^{-} \bar{\nu}_{\ell} \nu_{\tau}$ and jets from initial state radiation are present.

- $W+$ jets fakes

Events where one jet is misleadingly reconstructed as a lepton, i.e. jet "fakes" the signature of a lepton. The main source of such events is production of $W$ boson with associated production of at least 3 jets ( $W+\geq 3$ jets).

$Z+$ jets, and diboson events are generated using ALPGEN, PYTHIA and MADEVENT respectively, where PYTHIA is used to model parton showering and the underlying event for all generated samples [49-53]. CTEQ6.6 parton distribution functions (PDF) are used in all Monte Carlo simulations [54]. CDFSIM, a GEANT-based simulation, is used to model the CDF detector response $[55,56]$.

Events where $Z / \gamma^{*}$ decays to leptons and $Z \rightarrow \tau \tau$ events are generated using ALPGEN, and diboson events are generated with PYTHIA. All other processes pass selection by mis-identifying one or more leptons in the detector. These fake lepton events are dominated by $\mathrm{W}$ boson events with associated jets. Fakes are modeled by $\mathrm{W}+$ jets events where one jet passes selection criteria such that it is a good candidate to fake lepton selection.

The predicted number of events for each background process, along with the number of expected $t \bar{t}$ events at the measured cross section, is calculated from the measurement of the $t \bar{t}$ cross section in the dilepton decay channel [57]. The number of $t \bar{t}$ signal and backgrounds expected with the data corresponds to $5.1 \mathrm{fb}^{-1}$ are summarized in the Table 4.1.

Table 4.1: The number of expected events in data correspond to $5.1 \mathrm{fb}^{-1}$ with the observed number of events.

\begin{tabular}{lc} 
& CDF II Preliminary $\left(5.1 \mathrm{fb}^{-1}\right)$ \\
\hline \hline Process & Number of expected events \\
\hline$W W$ & $11.69 \pm 2.35$ \\
$W Z$ & $3.48 \pm 0.55$ \\
$Z Z$ & $2.25 \pm 1.75$ \\
Drell-Yan $(Z \rightarrow e e, \mu \mu)$ & $22.34 \pm 3.23$ \\
$Z \rightarrow \tau \tau$ & $12.21 \pm 2.17$ \\
Fakes & $34.27 \pm 9.46$ \\
$W \gamma$ & $0.42 \pm 0.44$ \\
\hline Total background & $86.24 \pm 14.05$ \\
$t \bar{t}(\sigma=7.4$ pb) & $236.97 \pm 11.29$ \\
\hline Total SM expectation & $323.21 \pm 25.12$ \\
Data $\left(5.1 \mathrm{fb}^{-1}\right)$ & 334 \\
\hline \hline
\end{tabular}




\subsection{Full Kinematical Reconstruction in Dilepton Chan- nel}

In this section, we describe a full kinematical reconstruction method we use in this analysis. By the event selection, we have two or more jets in events. we use the leading two energetic jets as $b$-jet and $\bar{b}$-jet candidates.

\subsubsection{Principle}

In a dilepton event, due to two undetected neutrinos in the final state, we have six unknown variables $\left(\vec{p}_{\nu}, \vec{p}_{\bar{\nu}}\right)$. Also, we have six constrains: $W^{+}$mass, $W^{-}$mass, top quark mass, anti-top quark mass, x-axial missing $E_{T}$ and y-axial missing $E_{T}$. The details of constrains are as follows.

$$
\begin{aligned}
M_{\ell^{+} \nu}^{2} & =\left(\left|\vec{p}_{\ell^{+}}\right|+\left|\vec{p}_{\nu}\right|\right)^{2}-\left(\vec{p}_{\ell^{+}}+\vec{p}_{\nu}\right)^{2}=M_{W}^{2} \\
M_{\ell^{-} \bar{\nu}}^{2} & =\left(\left|\vec{p}_{\ell^{-}}\right|+\left|\vec{p}_{\bar{\nu}}\right|\right)^{2}-\left(\vec{p}_{\ell^{-}}+\vec{p}_{\bar{\nu}}\right)^{2}=M_{W}^{2} \\
M_{\ell^{+} \nu b}^{2} & =\left(\left|\vec{p}_{\ell^{+}}\right|+\left|\vec{p}_{\nu}\right|+\left|\vec{p}_{b}\right|\right)^{2}-\left(\vec{p}_{\ell^{+}}+\vec{p}_{\nu}+\vec{p}_{b}\right)^{2}=M_{t}^{2} \\
M_{\ell^{-} \bar{\nu} \bar{b}}^{2} & =\left(\left|\vec{p}_{\ell^{-}}\right|+\left|\vec{p}_{\bar{\nu}}\right|+\left|\vec{p}_{\bar{b}}\right|\right)^{2}-\left(\vec{p}_{\ell^{-}}+\vec{p}_{\bar{\nu}}+\vec{p}_{\bar{b}}\right)^{2}=M_{t}^{2} \\
\left(\vec{p}_{\nu}+\vec{p}_{\bar{\nu}}\right)_{x} & =\left(E_{T}\right)_{x} \\
\left(\vec{p}_{\nu}+\vec{p}_{\bar{\nu}}\right)_{y} & =\left(E_{T}\right)_{y} .
\end{aligned}
$$

Typically we will have two or four possible solutions of $\left(\vec{p}_{\nu}, \vec{p}_{\bar{\nu}}\right)$ as well as two combinations due to $b-\bar{b}$ ambiguity in the system of the equation above. However without any additional redundant constraint, no one can tell which solution is the best solution.

The basic idea to select most likely solution is to calculate $p_{z}^{t \bar{t}}, p_{T}^{t \bar{t}}$ and $M_{t \bar{t}}$ for each solution and to look how plausible these reconstructed variables are. These variables, $p_{z}^{t \bar{t}}, p_{T}^{t \bar{t}}$ and $M_{t \bar{t}}$ are mostly distributed depending on the initial parton distributions which we can know to some extent in prior.

\subsubsection{Likelihood}

In the actual reconstruction method we adopt, we take resolution of $b$-jet energies and $E_{T}$ into account, i.e. the likelihood are given as a function of assumed $\vec{p}_{\nu}, \vec{p}_{\bar{\nu}}, E_{b}^{\text {guess }}$, and $E_{\bar{b}}^{\text {guess }}$. We use the following likelihood:

$$
\begin{aligned}
\mathcal{L}\left(\vec{p}_{\nu}, \vec{p}_{\bar{\nu}}, E_{b}^{\text {guess }}, E_{\bar{b}}^{\text {guess }}\right)=P\left(p_{z}^{t \bar{t}}\right) P\left(p_{T}^{t \bar{t}}\right) P\left(M_{t \bar{t}}\right) \times \\
\quad \frac{1}{\sigma_{\text {jet1 }}} \exp \left[-\frac{1}{2}\left\{\frac{E_{\text {jet1 }}^{\text {meas }}-E_{\text {jet1 }}^{\text {guess }}}{\sigma_{\text {jet1 }}}\right\}\right] \times \frac{1}{\sigma_{\text {jet2 }}} \exp \left[-\frac{1}{2}\left\{\frac{E_{\text {jet2 }}^{\text {meas }}-E_{\text {jet2 }}^{\text {guess }}}{\sigma_{\text {jet2 }}}\right\}\right] \times \\
\frac{1}{\sigma_{x}^{\text {MET }}} \exp \left[-\frac{1}{2}\left\{\frac{E_{T_{x}^{\text {meas }}}-E_{T_{x}^{\text {guess }}}}{\sigma_{x}^{\text {MET }}}\right\}\right] \times \frac{1}{\sigma_{y}^{\text {MET }}} \exp \left[-\frac{1}{2}\left\{\frac{E_{T}^{\text {meas }}-E_{T_{y}^{\text {guess }}}^{\text {gut }}}{\sigma_{y}^{\text {MET }}}\right\}\right],
\end{aligned}
$$

where $P\left(p_{z}^{t \bar{t}}\right), P\left(p_{T}^{t \bar{t}}\right)$ and $P\left(M_{t \bar{t}}\right)$ are probability density function of each variables in a $t \bar{t}$ candidate, which obtained from dilepton candidates in $t \bar{t}$ PYTHIA Monte Carlo sample. 

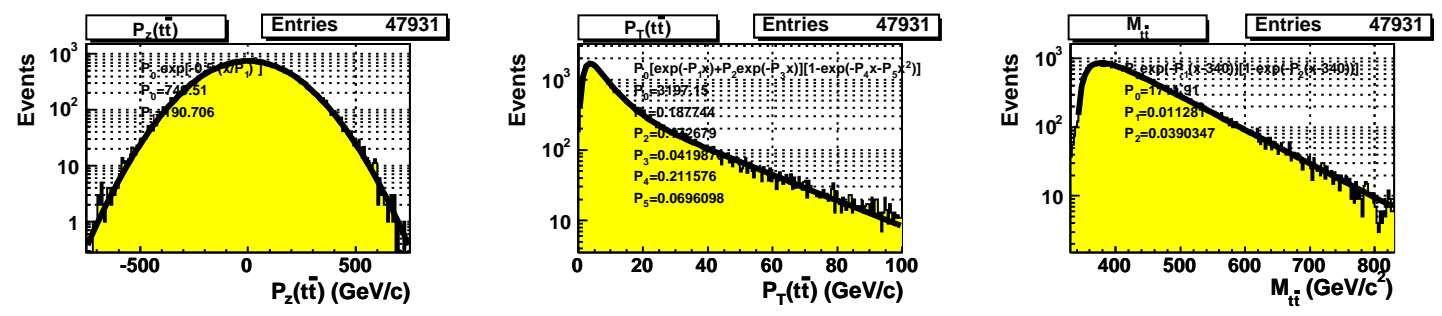

Figure 4.1: Distributions of $p_{z}^{t \bar{t}}, p_{T}^{t \bar{t}}$, and $M_{t \bar{t}}$ of dilepton candidates in $t \bar{t}$ PYTHIA Monte Carlo.

Figure 4.1 shows the distributions of $p_{z}^{t \bar{t}}, p_{T}^{t \bar{t}}$ and $M_{t \bar{t}}$ of dilepton candidates. Each distributions is fitted to an analytic function, and fit result and parameters are shown in the plots. In Equation (4.2), $E_{\text {jet } 1,2}^{\text {meas }}$ denote the measured energy of the jets which are assigned as $b$-jets and $\bar{b}$-jets, respectively. The measured energies of jets are corrected by a dilepton specific correction which is explained in the following sub section, after the Level5 jet energy correction. $E_{T x, y}^{\text {meas }}$ are $x, y$ components of the measured missing transverse energy. $\sigma_{\text {jet } 1,2}$ and $\sigma_{x, y}^{\mathrm{MET}}$ denote the resolution of measured jet energy and missing transverse energy which are also described in the Sec 4.4.3. $E_{\text {jet1,2 }}^{\text {guess }}$ and $E_{T \text { jet1,2 }}^{\text {guess }}$ in Equation (4.2) are the quantities deduced from the assumed $\vec{p}_{\nu}, \vec{p}_{\bar{\nu}}, E_{b}^{\text {guess }}$, and $E_{\bar{b}}^{\text {guess }}$, respectively. We take one representative set of $\left(\vec{p}_{\nu}, \vec{p}_{\bar{\nu}}, E_{b}^{\text {guess }}, E_{\bar{b}}^{\text {guess }}\right)$ which gives maximum likelihood in each event as reconstructed quantities. In both of two cases for $b-\bar{b}$ assignments, the likelihood is calculated and the assignment which gives the better likelihood ia chosen as the solution.

\subsubsection{Jet and MET Resolutions}

As mentioned in the previous subsection, we use corrected measured jet energies after the Level5 jet energy correction in the likelihood for the full kinematical reconstruction (Equation (4.2)) as well as the resolutions of the jet energies. The correction is performed so that the mean of $E_{\text {jet } 1,2}^{\text {meas }}$ goes to $b$-quark or $\bar{b}$-quark energy.

To obtain the correction factor and the resolution, we use reconstructed jets in dilepton candidates of $t \bar{t}$ Monte Carlo sample, which is matched to $b$-quark or $\bar{b}$-quark within $\Delta R<0.4$. We divide the matched jets into the following three pseudo-rapidity regions: $|\eta|<0.7,0.7<|\eta|<1.3$, and $1.3<|\eta|<2.5$. Then using the true $b(\bar{b})$-quark transverse energy $\left(E_{T}^{\text {true }}\right)$ and the observed transverse energy after Level5 correction $\left(E_{T}^{L 5}\right)$, we make the distribution of $\left(E_{T}^{\mathrm{true}}-E_{T}^{\mathrm{L} 5}\right) / E_{T}^{\mathrm{L} 5}$ for each $E_{T}^{\mathrm{L} 5}$. The plots in Figure 4.2 show mean and $\sigma$ of the distribution of $\left(E_{T}^{\text {true }}-E_{T}^{\mathrm{L} 5}\right) / E_{T}^{\mathrm{L} 5}$ as a function of $E_{T}^{\mathrm{L} 5}$ for each pseudorapidity region. Each plot in Figure 4.2 is fitted to the function $\exp \left(P_{0}+P_{1} x\right)+P_{2}$, and we use these parameters for the correction and the resolution in Equation (4.2).

For $E_{T}$, the resolutions are also used in the likelihood for the full kinematical reconstruction (Equation (4.2)). They are obtained by the $\sigma \mathrm{s}$ of the distributions of $E_{T}$ meas $E_{T x, y}^{\text {true }}$. Figure 4.3 shows the plot of $\sigma$ of $E_{T x, y}^{\text {meas }}-E_{T x, y}^{\text {true }}$ as a function of $E_{T}^{\text {jet1 }}+E_{T}^{\text {jet2 }}$. 

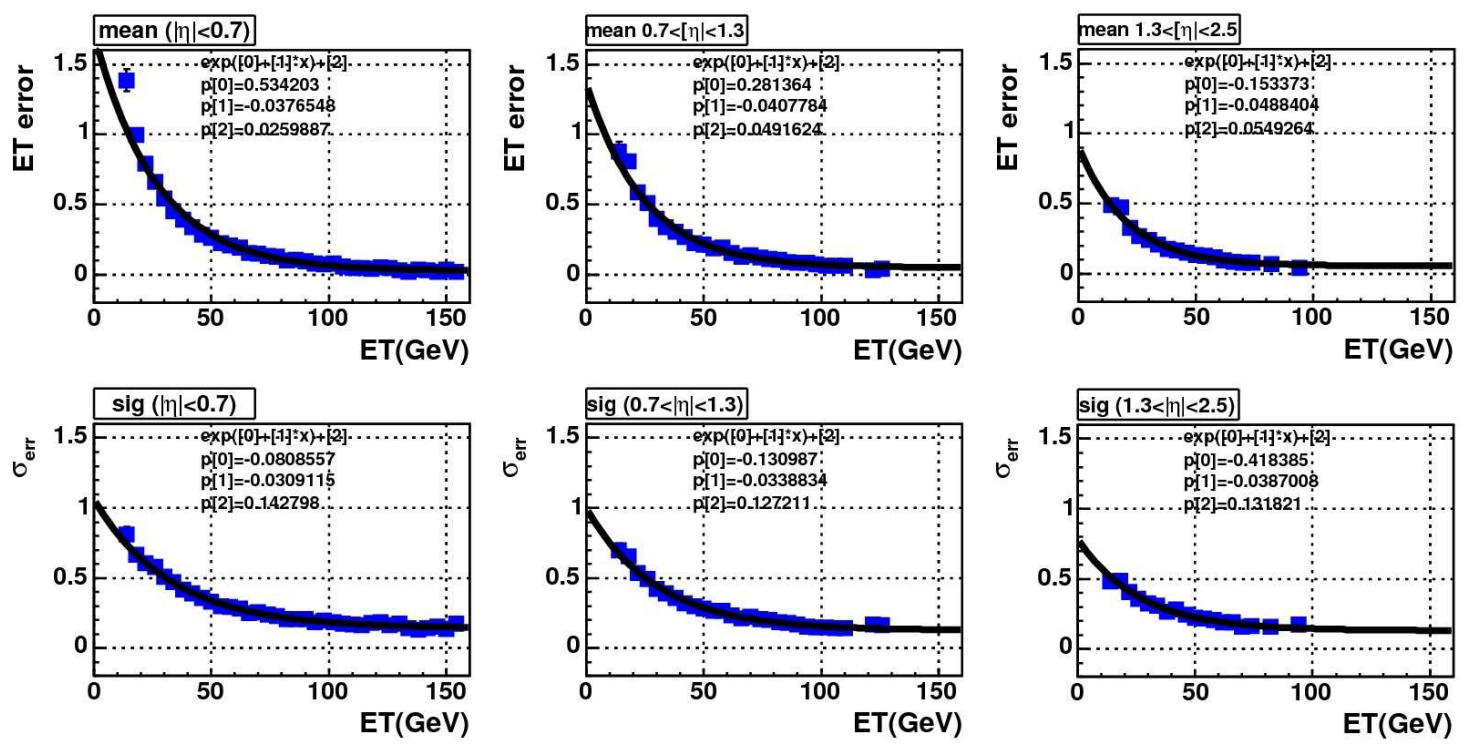

Figure 4.2: Mean and $\sigma$ of the distribution of $\left(E_{T}^{\text {true }}-E_{T}^{\mathrm{L} 5}\right) / E_{T}^{\mathrm{L} 5}$ as a function of $E_{T}^{\mathrm{L} 5}$ for jets matched within $\Delta R<0.4$ in dilepton candidates of Pythia Monte Carlo, for each region of $|\eta|<0.7,0.7<|\eta|<1.3$, and $1.3<|\eta|<2.5$.

\subsubsection{Performance of the Full Kinematical Reconstruction Method}

To check the performance of the full kinematical reconstruction method, we look at the scatter plots of generated values of kinematical variables versus reconstructed values. The full HEPG information, including the identity and kinematics of all particles in an event, is available in Monte Carlo samples. We use dilepton candidates where both of the two charged leptons in the events are matched to HEPG leptons within $\Delta R<0.1$ and both of the leading two jets are matched to $b$-quark of $\bar{b}$-quark within $\Delta R<0.4$ in $t \bar{t}$ PYTHIA Monte Carlo sample. The ratio of the lepton and jet matched events to the whole dilepton candidates is about $76 \%$. Three plots in Figure 4.4 show the scatter plots of $\cos \theta_{\text {beam }}$, $\cos \theta_{t}$, and $\cos \theta^{*}$, which are $\operatorname{cosine}$ of the angle of $\ell^{+}\left(\ell^{-}\right)$flight direction w.r.t. the beam axis in the top(anti-top) quark rest frame, cosine of the angle of top flight direction w.r.t. the proton direction in the $t \bar{t}$ rest frame, and cosine of the angle of $\ell^{+}\left(\ell^{-}\right)$flight direction in the $W^{+}\left(W^{-}\right)$rest frame w.r.t. $W^{+}\left(W^{-}\right)$flight direction in the top(anti-top) quark rest frame, respectively.

The events in the diagonal region in the scatter plots indicate that the variables are correctly reconstructed, while the events in the off-diagonal region indicate that the variables are incorrectly reconstructed. We can see bands of events in the diagonal regions in the scatter plots, which indicate the method reasonably successfully reconstructs events including neutrinos as well as $b$ and $\bar{b}$ assignment.

The ratio of the events where $b$ and $\bar{b}$ are correctly assigned to the two jets by the full kinematical reconstructed method to the lepton and jet matched dilepton candidate is found to be $75 \%$. 


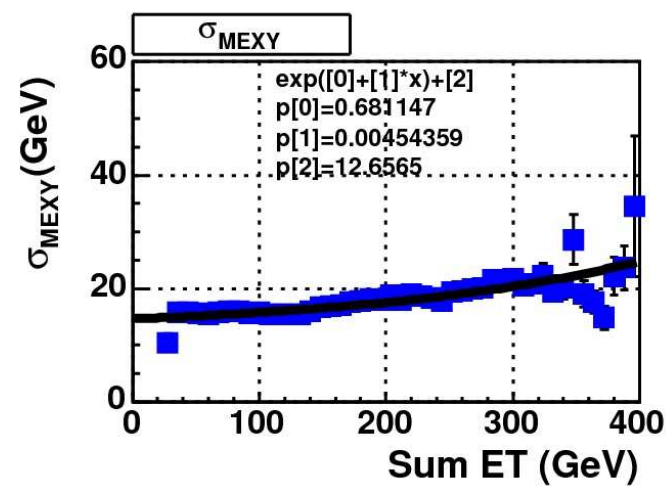

Figure 4.3: $\sigma$ of the distribution of $E_{x, y}^{\text {meas }}-E_{x, y}^{\text {true }}$ as a function of $E_{T}^{\text {jet1 }}+E_{T}^{\text {jet2 }}$ for dilepton candidates of Pythia Monte Carlo.
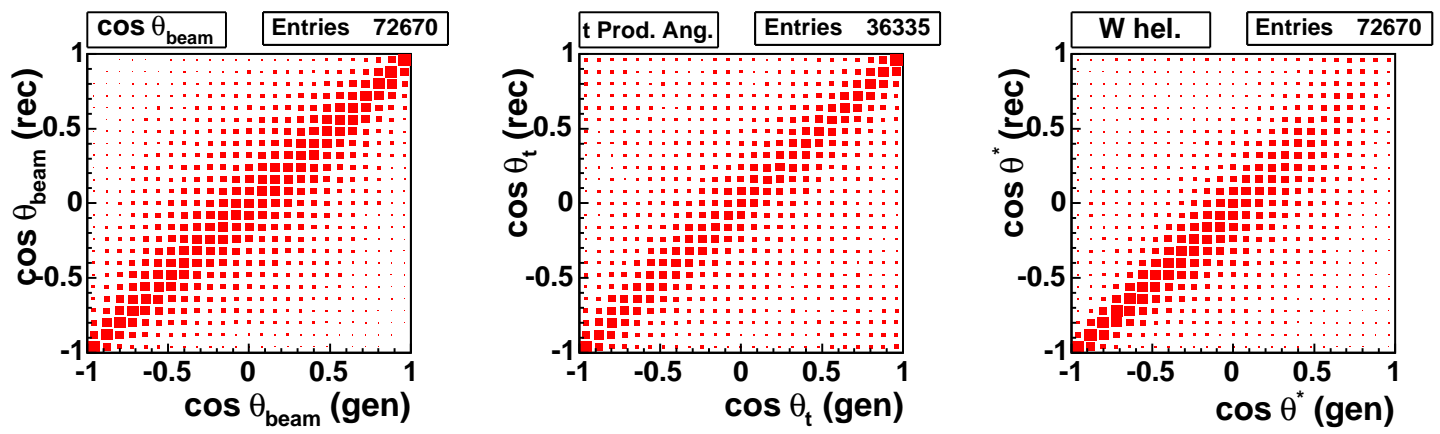

Figure 4.4: Scatter plots of reconstructed kinematical variables versus the true value of the corresponding variables. The kinematical variables are $\cos \theta_{\text {beam }}($ left $), \cos \theta_{t}$ (middle), and $\cos \theta^{*}$ (right). The areas of the square are proportional to the entries in the bins. For each plot, we use dilepton candidates which satisfy following two requirements. Both of the two reconstructed charged leptons obtained from the full kinematical reconstruction are matched to HEPG charged leptons within $\Delta R<0.1$, respectively. Both of the two reconstructed leading jets obtained from the full kinematical reconstruction are matched to HEPG $b$-quark or HEPG $\bar{b}$-quark within $\Delta R<0.4$, respectively. 


\subsection{Templates of the Signal and the Background}

In this section, we discuss about the two dimensional distribution of reconstructed $\cos \theta_{+^{-}}$ $\cos \theta_{-}$and $\cos \theta_{b}-\cos \theta_{\bar{b}}$ of the $t \bar{t}$ signal and background. We use these expected distribution as templates to extract measured $\kappa$ from observed reconstructed distribution by likelihood fitting. Therefore, for the $t \bar{t}$ signal, the templates should be as a function fo any assumed $\kappa$.

\subsubsection{Template Fit Function}

To obtain the templates as an analytic function, $f(x, y)$, we assume as follows:

- Polynomial function of $x$ and $y$ up to $O\left((x, y)^{4}\right)$.

$$
f(x, y)=\sum_{i+j \leq 4} C_{i j} x^{i} y^{j}
$$

- Assume $P$ is conserved. On this assumption, physical quantities at $\cos \theta_{ \pm}$are equivalent to ones at $-\cos \theta_{ \pm}$under $\mathrm{P}$ reversal(See Appendix B), ie. we assume

$$
f(x, y)=f(-x,-y)
$$

- Assume $C P$ is conserved. $\cos \theta_{ \pm}$change to $\cos \theta_{\mp}$ under $C P$ reversal(See Appendix B), i.e. we assume

$$
f(x, y)=f(y, x)
$$

On the assumption above, we obtain

$$
\begin{aligned}
f(x, y)=\frac{C_{0}}{4}\{1 & -C_{1}-C_{3}-C_{5}+3 C_{1} \frac{x^{2}+y^{2}}{2} \\
& \left.+C_{2} x y+5 C_{3} \frac{x^{4}+y^{4}}{2}+C_{4} \frac{x^{3} y+x y^{3}}{2}+9 C_{5} x^{2} y^{2}\right\}
\end{aligned}
$$

and

$$
\int_{-1}^{1} d x \int_{-1}^{1} d y f(x, y)=C_{0} .
$$

Hereafter we suppose $C_{0}=1$ unless it's specified.

\subsubsection{Signal Template as a Function of $\kappa$}

We use a $t \bar{t}$ Monte Carlo sample generated by Pythia with $M_{t}=172.5 \mathrm{GeV} / c^{2}$. In this sample, there is no spin correlation between generated $t$ and $\bar{t}$. We put a weight, $\left(1+\kappa \cos \theta_{+}^{\text {hepg }} \cos \theta_{-}^{\text {hepg }}\right) /(1+|\kappa|)$, on a dilepton candidate in its Monte Carlo sample, where $\kappa$ is assumed spin-spin correlation coefficient in the beamline basis and $\cos \theta_{ \pm}^{\text {hepg }}$ 

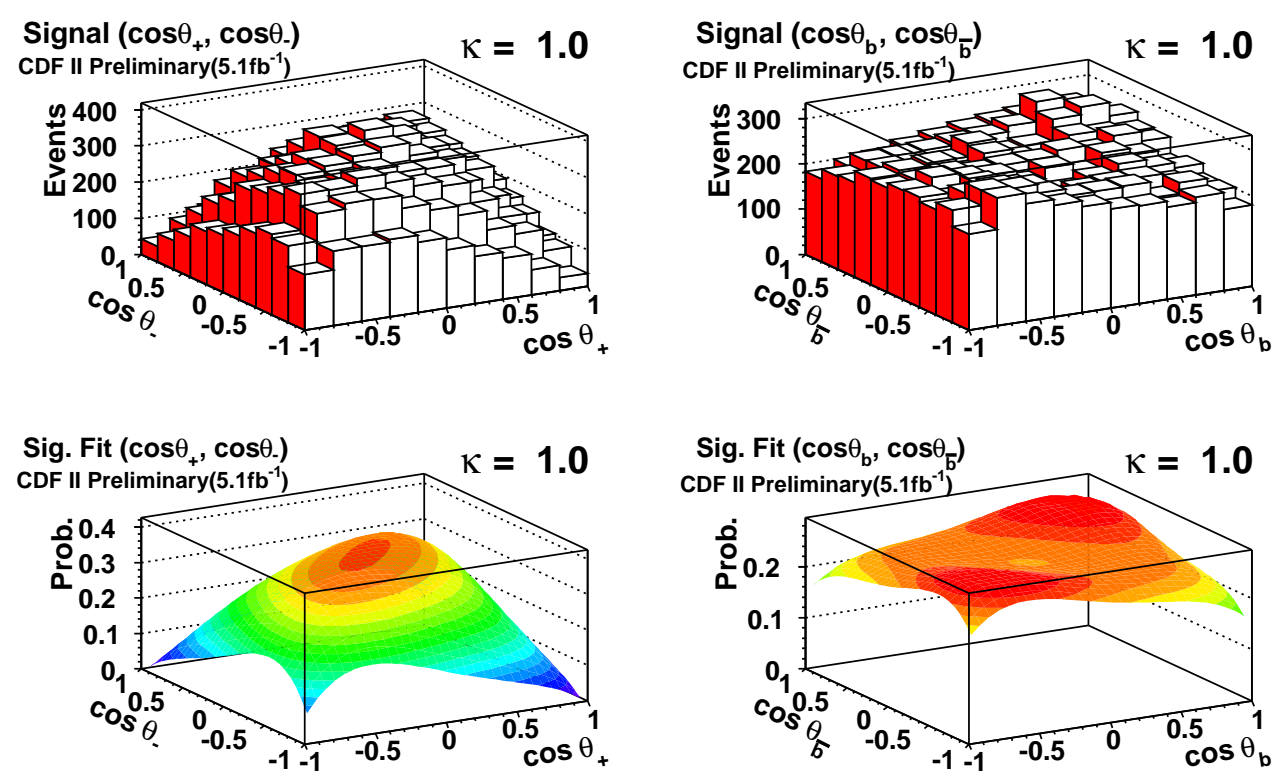

Figure 4.5: The distributions of reconstructed $\left(\cos \theta_{+}, \cos \theta_{-}\right)$(left) and $\left(\cos \theta_{b}, \cos \theta_{\bar{b}}\right)$ (right) of weighted dilepton candidates in $t \bar{t}$ Pythia Monte Carlo sample on the assumption of $\kappa=+1$, and their fit results.

represent true $\cos \theta_{ \pm}$using HEPG information. Then, we obtain the reconstructed distributions of $\left(\cos \theta_{+}, \cos \theta_{-}\right)$and $\left(\cos \theta_{b}, \cos \theta_{\bar{b}}\right)$ for dilepton candidates from $t \bar{t}$ signal with an assumed $\kappa$.

We made the 10 by 10 bin distributions of reconstructed $\left(\cos \theta_{+}, \cos \theta_{-}\right)$and $\left(\cos \theta_{b}, \cos \theta_{\bar{b}}\right)$ from weighted dilepton candidates in $t \bar{t}$ Pythia Monte Carlo sample with assumed $\kappa$ ranging from -1 to 1 with 0.2 step. For each distribution we fit the resultant distribution to the fit function of Equation (4.6), and obtain fit parameters $C_{i}^{\ell}(i=1, \cdots, 5)$ for leptons, and $C_{i}^{b}(i=1, \cdots, 5)$ for $b$-jets as a function of $\kappa$.

Figure 4.5, 4.6 and 4.5 shows the reconstructed distributions and their fit results in cases of $\kappa=+1, \kappa=0$, and $\kappa=-1$, respectively.

Figure 4.8 and 4.9 show $\kappa$ dependence of the fit parameters $C_{i}^{\ell}$ and $C_{i}^{b}$, respectively.

Since the differential cross-section corresponding to each events a linear function of $\kappa$, each parameter except for normalization parameter $C_{0}$ should be a linear function of $\kappa$ as well.

We fit $C_{i}^{\ell}(\kappa)$ and $C_{i}^{b}(\kappa)(i=1, \cdots, 5)$ to a linear function of $\kappa$ so than we can describe signal templates with any $\kappa$ assumed. 

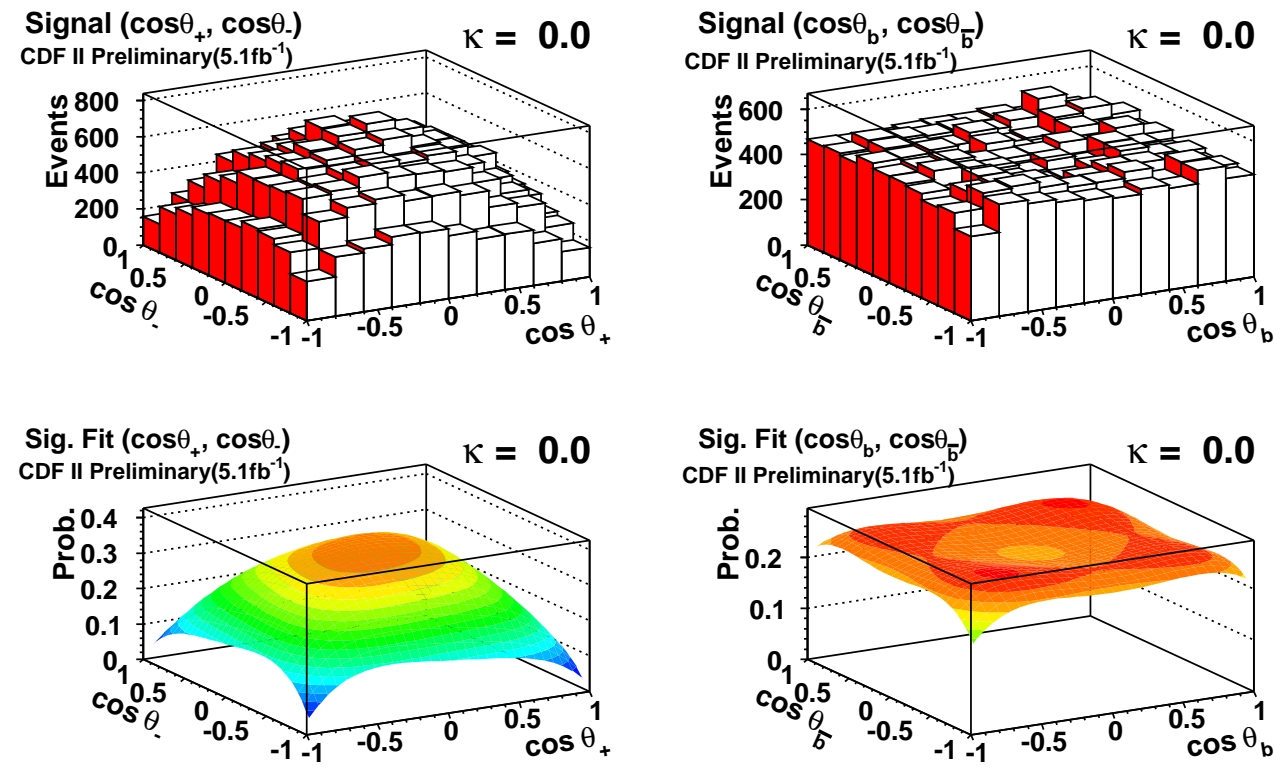

Figure 4.6: The distributions of reconstructed $\left(\cos \theta_{+}, \cos \theta_{-}\right)$(left) and $\left(\cos \theta_{b}, \cos \theta_{\bar{b}}\right)$ (right) of dilepton candidates in $t \bar{t}$ Pythia Monte Carlo sample on the assumption of $\kappa=0$, and their fit results.
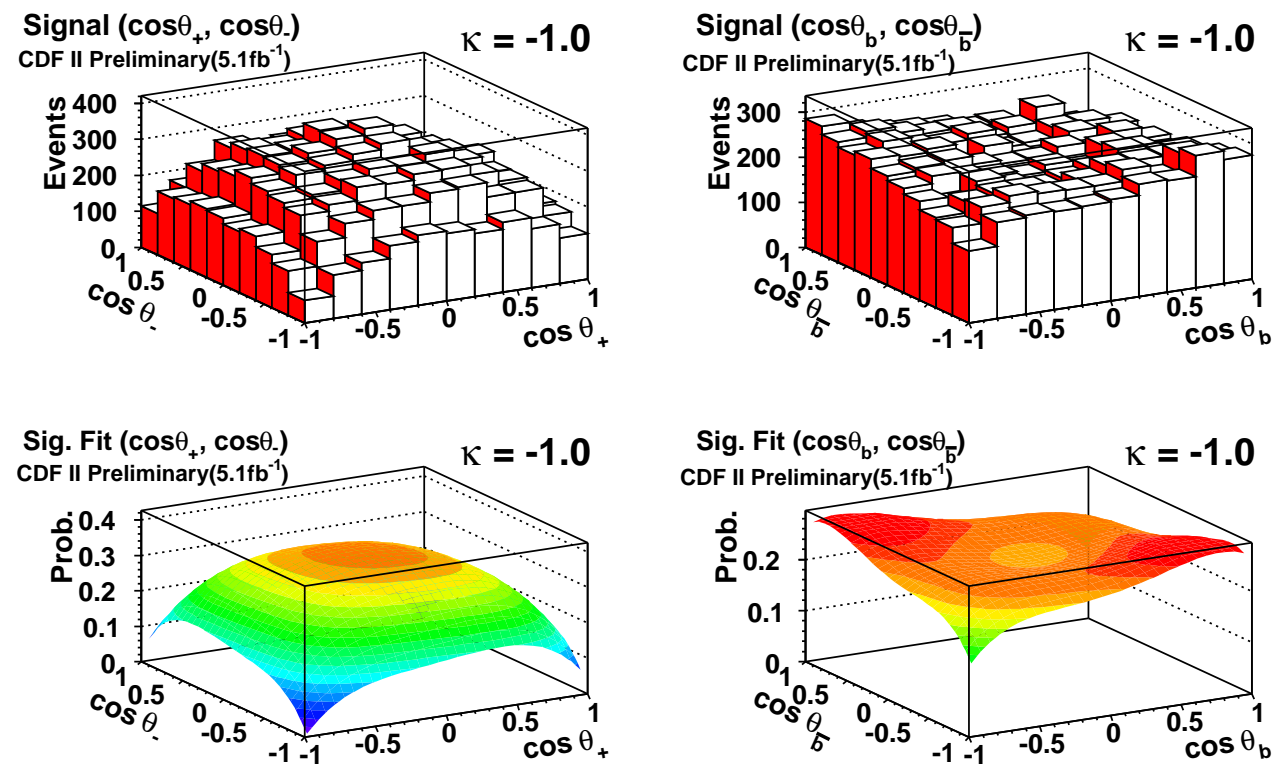

Figure 4.7: The distributions of reconstructed $\left(\cos \theta_{+}, \cos \theta_{-}\right)$(left) and $\left(\cos \theta_{b}, \cos \theta_{\bar{b}}\right)($ right $)$ of weighted dilepton candidates in $t \bar{t}$ Pythia Monte Carlo sample on the assumption of $\kappa=-1$. The two surfaces shown in lower row are fit results of the distributions above. 

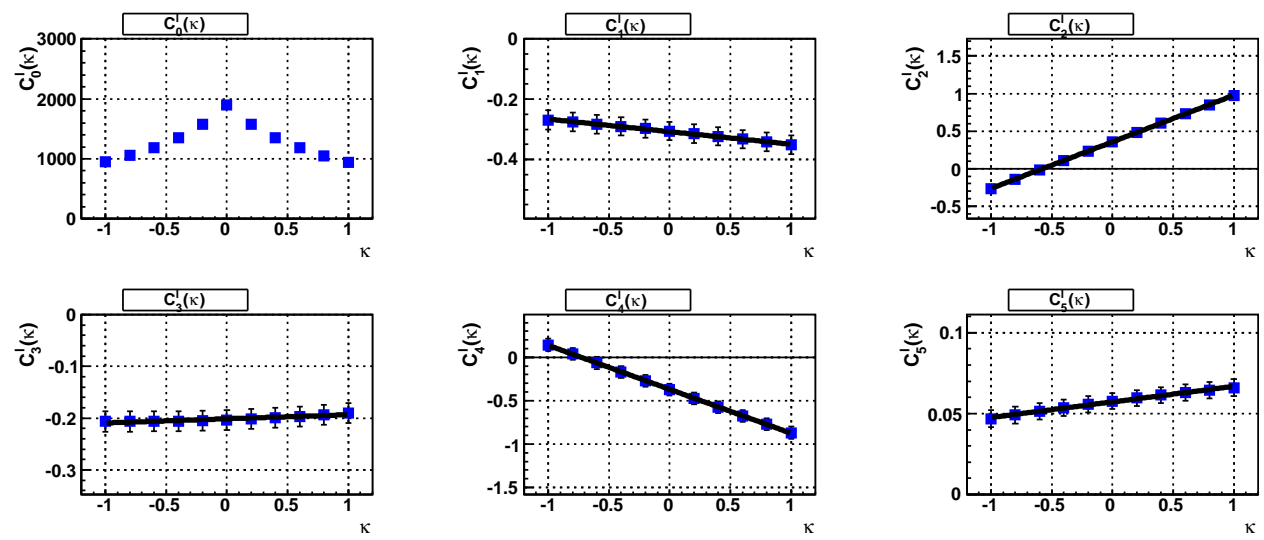

Figure 4.8: $\kappa$ dependence of the fit parameters $\left(C_{i}^{\ell}(\kappa)\right)$ for the $\left(\cos \theta_{+}, \cos \theta_{-}\right)$distribution. We assume $C_{i}^{\ell}(\kappa)(i=1, \cdots, 5)$ are a linear function of $\kappa$.
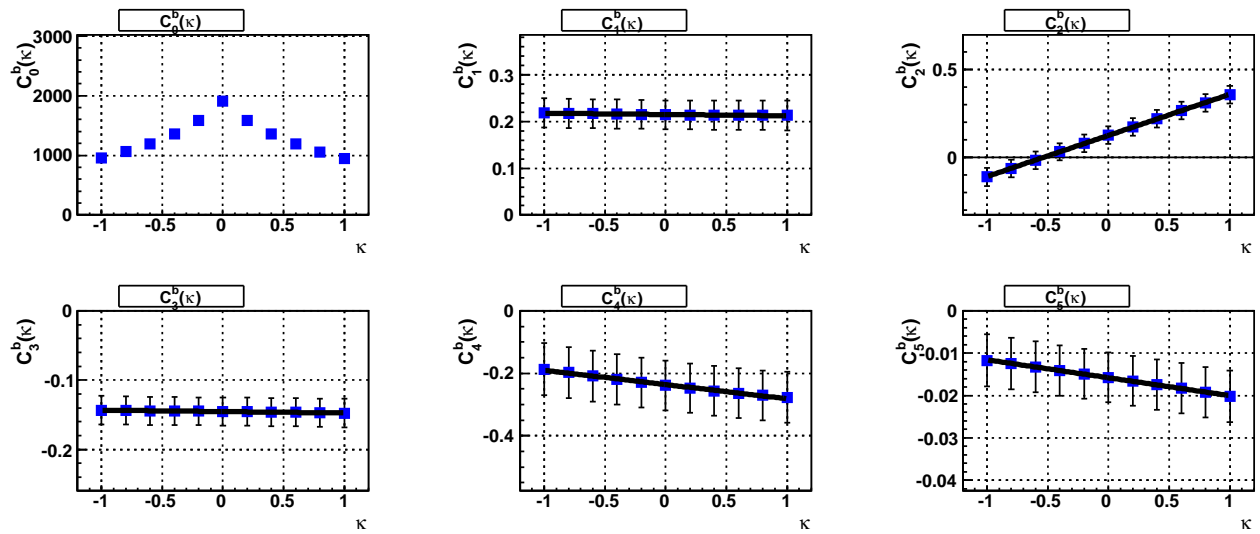

Figure 4.9: $\kappa$ dependence of the fit parameters $\left(C_{i}^{b}(\kappa)\right)$ for the $\left(\cos \theta_{b}, \cos \theta_{\bar{b}}\right)$ distribution. We assume $C_{i}^{b}(\kappa)(i=1, \cdots, 5)$ are a linear function of $\kappa$. 


\subsubsection{Background Template}

Next, we describe background templates. We use the table 4.1 as expected number of background. We consider diboson ( $W W, W Z$, and $Z Z)$, Drell-Yan $\left(Z / \gamma^{*} \rightarrow e e, \mu \mu\right), Z \rightarrow$ $\tau \tau$, and fake ( $W+\mathrm{QCD}$ jet, where a jet is misidentified as a lepton) processes as background events. We neglect $W \gamma$ process, since it is found to be very small contribution.

For diboson, Drell-Yan, and $Z \rightarrow \tau \tau$ backgrounds, we estimate the background distributions using Monte Carlo basically. For fake backgrounds, we use real data to estimate the background distributions.

\section{Diboson}

We use $W W / W Z / Z Z$ Monte Carlo samples generated by Pythia event generator to estimate diboson background. We made the distribution of reconstructed $\left(\cos \theta_{+}, \cos \theta_{-}\right)$ and $\left(\cos \theta_{b}, \cos \theta_{\bar{b}}\right)$ of dilepton candidates in $W W, W Z$ and $Z Z$ simlated samples, respectively. Then, we normalize the distributions for each sample to each expected number of events and accumulate them. Figure 4.10 shows the distributions of reconstructed $\left(\cos \theta_{+}, \cos \theta_{-}\right)$and $\left(\cos \theta_{b}, \cos \theta_{\bar{b}}\right)$ of dilepton candidates in $W W / W Z / Z Z$ samples. The right column plots in the figure indicate the magnitude of $1 \sigma$ uncertainty of each bin of the left column distributions.
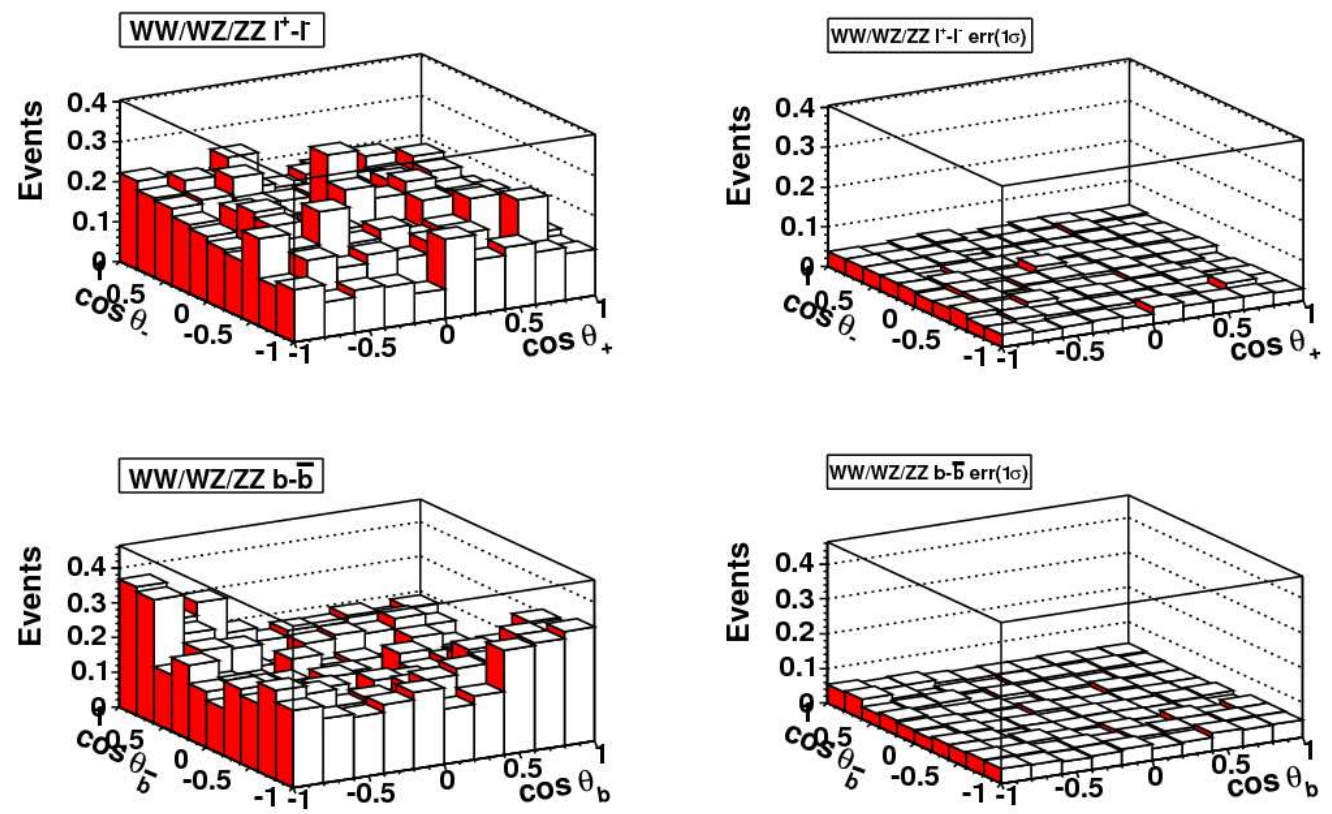

Figure 4.10: The distributions of reconstructed $\left(\cos \theta_{+}, \cos \theta_{-}\right)$(upper) and $\left(\cos \theta_{b}, \cos \theta_{\bar{b}}\right)$ (lower) of dilepton candidates in $W W / W Z / Z Z$ simulated samples. The distribution in the right column indicates the magnitude of $1 \sigma$ uncertainty of each bin of the left distribution. 
We use Monte Carlo samples of $Z / \gamma^{*}+n$ partons where $Z / \gamma^{*} \rightarrow \tau \tau$ which are generated by ALPGEN event generator to estimate $Z \rightarrow \tau \tau$ background.

Table 4.2 shows processes in $Z \rightarrow \tau \tau$ background, number of dilepton candidates found in Monte Carlo of each process divided by generated number of events and crosssection in $\mathrm{pb}$ for each process. We accumulate distributions from each process with appropriate weight considering its acceptance and cross-section, then finally normalize the accumulated distribution to expected number of event of $Z \rightarrow \tau \tau$ background.

Table 4.2: Table of processes in $Z \rightarrow \tau \tau$ background. Number of dilepton candidates found in Monte Carlo of each process divided by generated number of events and crosssection in $\mathrm{pb}$ for each process are also shown.

\begin{tabular}{lrr}
\hline \hline Process & \# of candidate/\# of generated & X-section[pb] \\
\hline$Z$ peak & & \\
$Z+0$ parton & $0 / 7.8 \mathrm{M}$ & 157.7 \\
$Z+1$ parton & $47 / 7.8 \mathrm{M}$ & 21.5 \\
$Z+\geq 2$ p partons & $1045 / 3.05 \mathrm{M}$ & 4.14 \\
\hline$M_{Z}[20-75]$ & & \\
$Z+0$ parton & $0 / 1.45 \mathrm{M}$ & 160.0 \\
$Z+1$ parton & $1 / 1.47 \mathrm{M}$ & 8.38 \\
$Z+\geq 2$ partons & $78 / 2.90 \mathrm{M}$ & 1.82 \\
\hline$M_{Z}[105-800]$ & & \\
$Z+0$ parton & $0 / 350 \mathrm{k}$ & 4.07 \\
$Z+1$ parton & $30 / 261 \mathrm{k}$ & 0.706 \\
$Z+2$ partons & $401 / 346 \mathrm{k}$ & 0.117 \\
$Z+3$ partons & $825 / 350 \mathrm{k}$ & 0.0185 \\
$Z+\geq 4$ partons & $206 / 71.7 \mathrm{k}$ & 0.0033 \\
\hline \hline
\end{tabular}

Figure 4.11 shows the distributions of reconstructed $\left(\cos \theta_{+}, \cos \theta_{-}\right)$and $\left(\cos \theta_{b}, \cos \theta_{\bar{b}}\right)$ of dilepton candidates in $Z \rightarrow \tau \tau$ samples.

\section{Drell-Yan}

We use Monte Carlo samples of $Z / \gamma^{*}+n$ partons where $Z / \gamma^{*} \rightarrow e e, \mu \mu$ which are generated by ALPGEN event generator to estimate Drell-Yan background.

Table 4.3 and 4.4 shows processes in Drell-Yan background, number of dilepton candidates found in Monte Carlo of each process divided by generated number of events and cross-section in $\mathrm{pb}$ for each process. We accumulate distributions from each process with appropriate weight considering its acceptance and cross-section, then finally normalize the accumulated distribution to expected number of events of Drell-Yan background.

Figure 4.12 shows the distributions of reconstructed $\left(\cos \theta_{+}, \cos \theta_{-}\right)$and $\left(\cos \theta_{b}, \cos \theta_{\bar{b}}\right)$ of dilepton candidates in $Z / \gamma^{*} \rightarrow e e, \mu \mu$ simulated samples. 

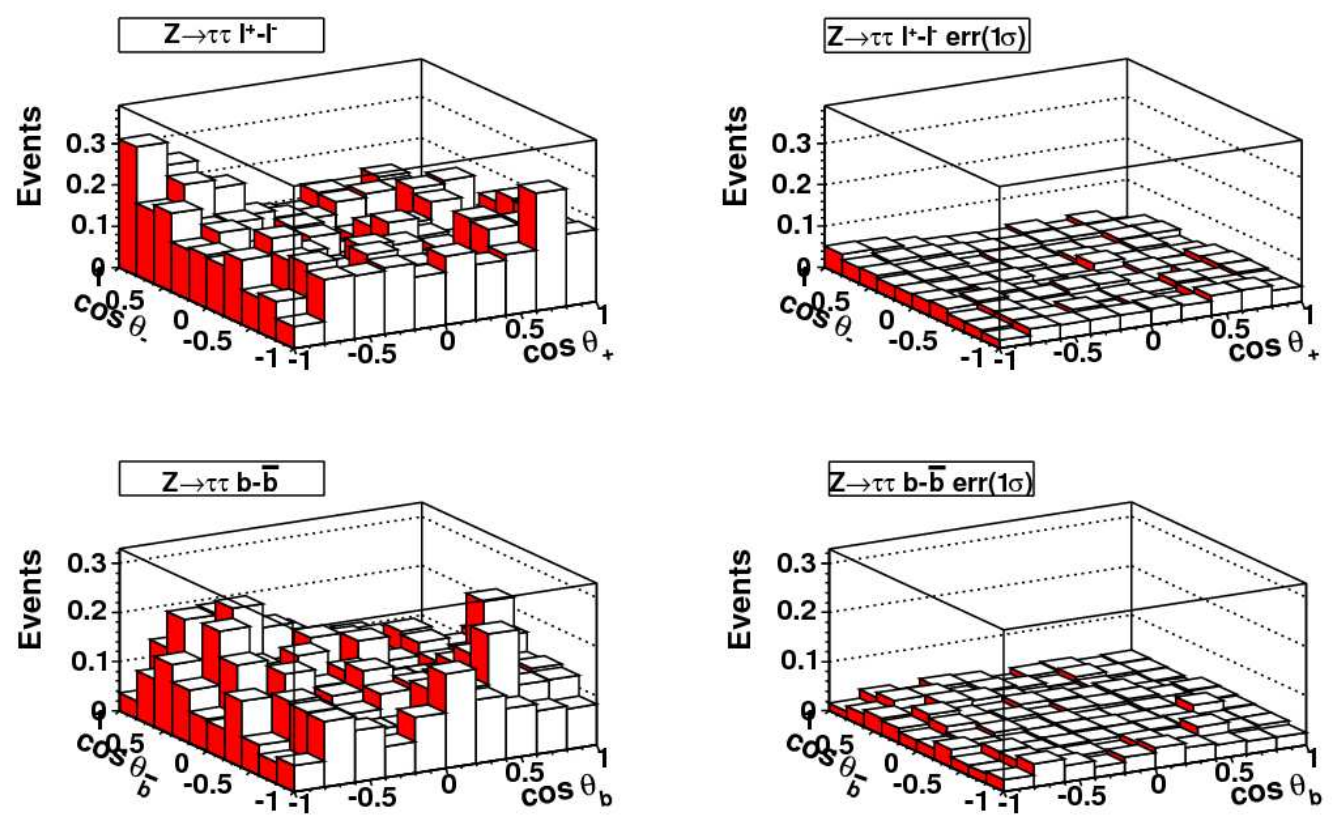

Figure 4.11: The distributions of reconstructed $\left(\cos \theta_{+}, \cos \theta_{-}\right)$(upper) and $\left(\cos \theta_{b}, \cos \theta_{\bar{b}}\right)$ (lower) of dilepton candidates in $Z \rightarrow \tau \tau$ simulated samples. The distributions in the right column indicates the magnitude of $1 \sigma$ uncertainty of each bin of the left distribution.

\section{Fake}

For fake events where a jet fakes charged lepton, we hardly estimate this background with Monte Carlo simulation. Instead, we use data to estimate the fake background, and validate the fake background using same sign dilepton events where the contribution from fake background is dominated. We pick up an event have high- $p_{\mathrm{T}}$ lepton $(e$ or $\mu)$ and at least one jet which can fake lepton from $5.1 \mathrm{fb}^{-1}$ data, and forcibly fake the jet to an electron or muon, then set an event weight for the event with faked lepton by its fake rate. The fake rate is a lepton type dependent probability that a fakeable object, that is an object which shares some of the jets and some of the high $p_{T}$ lepton characteristics, can be reconstructed as a good lepton. The fake rate is calculated in [57].

Subsequently we apply the selection to the event and perform the kinematical reconstruction to make distributions of dilepton candidates, taking the weight of each event into account, and finally normalize the distributions to expected number of events of fake background. Figure 4.13 shows the distributions of reconstructed $\left(\cos \theta_{+}, \cos \theta_{-}\right)$ and $\left(\cos \theta_{b}, \cos \theta_{\bar{b}}\right)$ of dilepton candidates obtained from lepton + fakeable jet events in $5.1 \mathrm{fb}^{-1}$ data.

The right column plots in the figure indicate the magnitude of $1 \sigma$ uncertainty of each bin of the left distribution.

Peaky bins in these plots come from single events with high fake rate jet (typically low- $p_{\mathrm{T}}$ fakeable jet), therefore have large statistical uncertainties. 
Table 4.3: Table of processes in Drell-Yan background. Number of dilepton candidates found in Monte Carlo of each process divided by generated number of events and crosssection in $\mathrm{pb}$ for each process are also shown.

\begin{tabular}{lcr}
\hline \hline Process & \# of candidate/\# of generated & X-section[pb] \\
\hline$M_{e e}[75-105]$ & & \\
$e e+0$ parton & $0 / 3.46 \mathrm{M}$ & 157.7 \\
$e e+1$ parton & $25 / 3.45 \mathrm{M}$ & 21.6 \\
$e e+2$ partons & $801 / 6.07 \mathrm{M}$ & 3.46 \\
$e e+3$ partons & $218 / 678 \mathrm{k}$ & 0.550 \\
$e e+\geq 4$ partons & $393 / 675 \mathrm{k}$ & 0.0990 \\
\hline$M_{e e}[8-20]$ & & \\
$e e+0$ parton & $0 / 682 \mathrm{k}$ & 1512.4 \\
$e e+1$ parton & $0 / 682 \mathrm{k}$ & 19.7 \\
$e e+\geq 2$ partons & $3 / 671 \mathrm{k}$ & 6.97 \\
\hline$M_{e e}[20-75]$ & & \\
$e e+0$ parton & $0 / 686 \mathrm{~K}$ & 160.0 \\
$e e+1$ parton & $3 / 666 \mathrm{~K}$ & 8.38 \\
$e e+2$ partons & $726 / 6.06 \mathrm{M}$ & 1.60 \\
$e e+3$ partons & $344 / 675 \mathrm{k}$ & 0.233 \\
$e e+\geq 4$ partons & $788 / 671 \mathrm{k}$ & 0.0398 \\
\hline$M_{e e}[105-600]$ & & \\
$e e+0$ parton & $7 / 669 \mathrm{k}$ & 4.07 \\
$e e+1$ parton & $166 / 675 \mathrm{k}$ & 0.706 \\
$e e+2$ partons & $1395 / 664 \mathrm{k}$ & 0.117 \\
$e e+3$ partons & $3528 / 681 \mathrm{k}$ & 0.0185 \\
$e e+\geq 4 \mathrm{p}$ partons & $6071 / 679 \mathrm{k}$ & 0.00333 \\
\hline \hline
\end{tabular}

\section{All Background}

Finally we accumulate distributions from each component of background: $W W / W Z / Z Z$, $Z \rightarrow \tau \tau, Z / \gamma^{*} \rightarrow e e, \mu \mu$, and fake samples.

Figure 4.14 show the resulting distributions of reconstructed $\left(\cos \theta_{+}, \cos \theta_{-}\right)$and $\left(\cos \theta_{b}, \cos \theta_{\bar{b}}\right)$ of accumulated dilepton candidates from all components discussed above. The distribution in the right column indicates the magnitude of $1 \sigma$ uncertainty of each bin of the upper distribution.

The distributions indicate no significant $P$ violating component. Hence we adopt the same fit function form (Equation 4.6) as the signal templates to fit the distributions. The surfaces in the lower row are fit results. The $\chi^{2} /$ ndf's of fit are found to 100.761/94 for $\left(\cos \theta_{+}, \cos \theta_{-}\right)$distribution and 94.9453/94 for $\left(\cos \theta_{b}, \cos \theta_{\bar{b}}\right)$ distribution, which are corresponding to $29.8 \%$ and $45.3 \%$ for $\chi^{2}$ probabilities, respectively. 
Table 4.4: $\mu \mu$ channels of Drell-Yan background.

\begin{tabular}{lcr}
\hline \hline Process & \# of candidate/\# of generated & X-section[pb] \\
\hline$M_{\mu \mu}$ [75-105] & & \\
$\mu \mu+0$ parton & $1 / 3.48 \mathrm{M}$ & 157.7 \\
$\mu \mu+1$ parton & $31 / 3.48 \mathrm{M}$ & 21.6 \\
$\mu \mu+2$ partons & $970 / 6.14 \mathrm{M}$ & 3.46 \\
$\mu \mu+3$ partons & $268 / 686 \mathrm{k}$ & 0.548 \\
$\mu \mu+\geq 4 \mathrm{p}$ partons & $480 / 685 \mathrm{k}$ & 0.0994 \\
\hline$M_{\mu \mu}[8-20]$ & & \\
$\mu \mu+0$ parton & $0 / 682 \mathrm{k}$ & 1512.6 \\
$\mu \mu+1$ parton & $2 / 677 \mathrm{k}$ & 19.7 \\
$\mu \mu+\geq 2$ partons & $13 / 682 \mathrm{k}$ & 6.98 \\
\hline$M_{\mu \mu}[20-75]$ & & \\
$\mu \mu+0$ parton & $0 / 676 \mathrm{k}$ & 160.0 \\
$\mu \mu+1$ parton & $3 / 681 \mathrm{k}$ & 8.39 \\
$\mu \mu+2$ partons & $864 / 6.07 \mathrm{M}$ & 1.60 \\
$\mu \mu+3$ partons & $352 / 675 \mathrm{k}$ & 0.233 \\
$\mu \mu+\geq 4$ partons & $967 / 681 \mathrm{k}$ & 0.398 \\
\hline$M_{\mu \mu}[105-600]$ & & \\
$\mu \mu+0$ parton & $16 / 686 \mathrm{k}$ & 4.07 \\
$\mu \mu+1$ parton & $213 / 683 \mathrm{k}$ & 0.706 \\
$\mu \mu+2$ partons & $1997 / 683 \mathrm{k}$ & 0.117 \\
$\mu \mu+3$ partons & $4071 / 671 \mathrm{k}$ & 0.0185 \\
$\mu \mu+\geq 4$ partons & $6822 / 681 \mathrm{k}$ & 0.00332 \\
\hline \hline
\end{tabular}



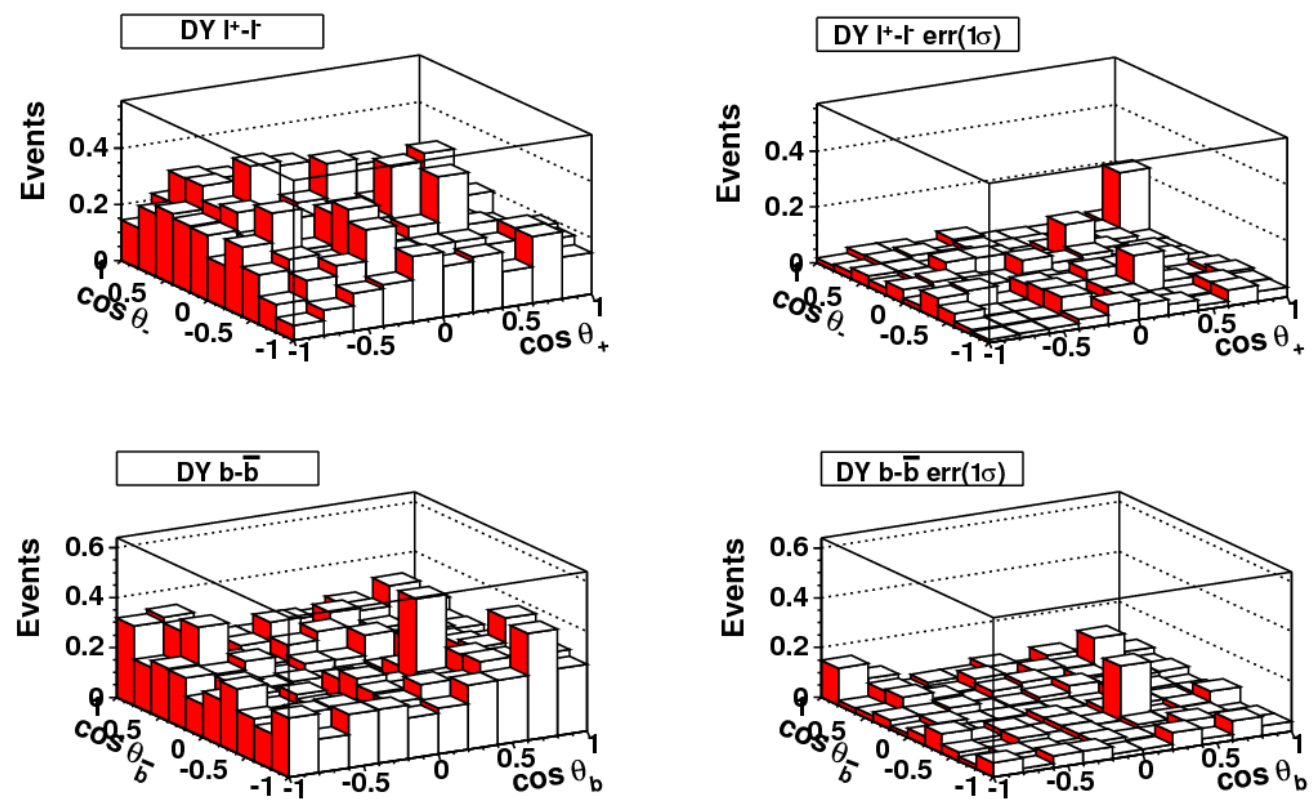

Figure 4.12: The distributions of reconstructed $\left(\cos \theta_{+}, \cos \theta_{-}\right)$(upper) and $\left(\cos \theta_{b}, \cos \theta_{\bar{b}}\right)$ (lower) of dilepton candidates in $Z / \gamma^{*} \rightarrow e e, \mu \mu$ simulated samples. The distribution in the right column indicates the magnitude of $1 \sigma$ uncertainty of each bin of the left distribution.
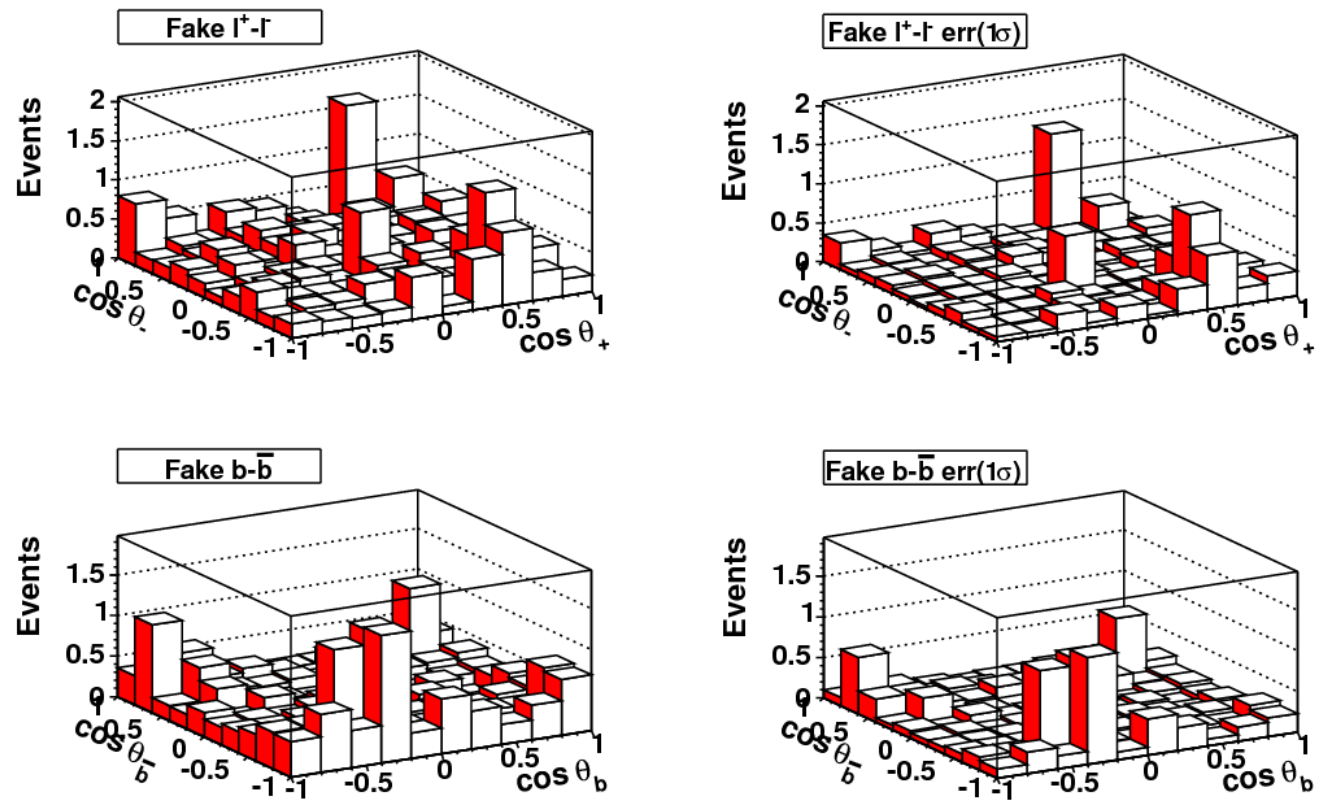

Figure 4.13: The distributions of reconstructed $\left(\cos \theta_{+}, \cos \theta_{-}\right)$(upper) and $\left(\cos \theta_{b}, \cos \theta_{\bar{b}}\right)$ (lower) of fake dilepton candidates obtained from lepton + fakeable jet events in $5.1 \mathrm{fb}^{-1}$ data. The distribution in the right column indicates the magnitude of $1 \sigma$ uncertainty of each bin of the left distribution. 

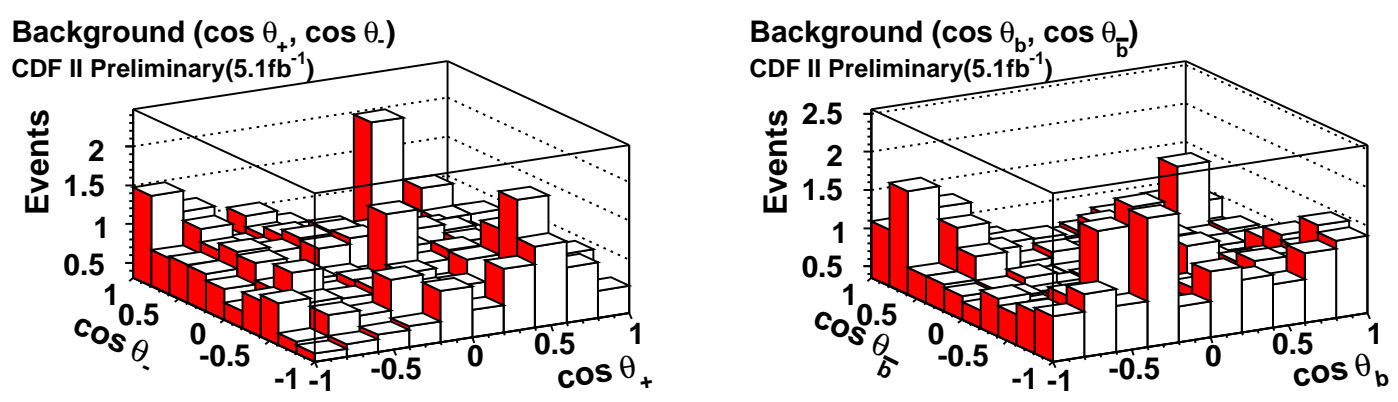

Bkg. $1 \sigma$ Err. $\left(\cos \theta_{,}, \cos \theta_{-}\right)$

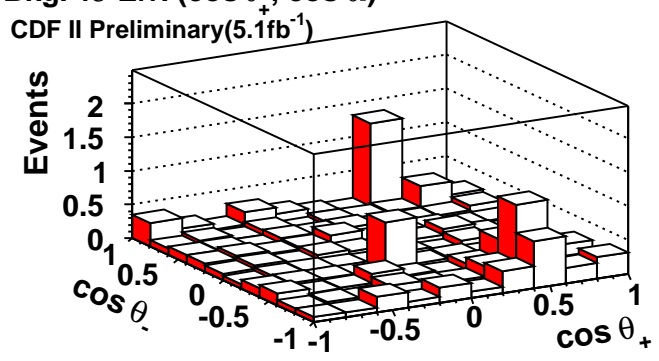

Bkg. $1 \sigma$ Err. $\left(\cos \theta_{b}, \cos \theta_{\bar{b}}\right)$

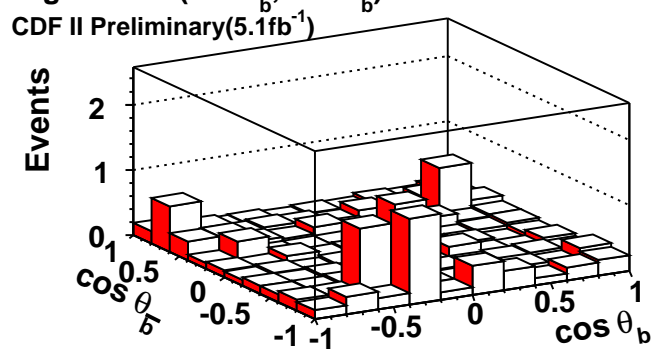

Bkg. Fit $\left(\cos \theta_{+}, \cos \theta_{-}\right)$
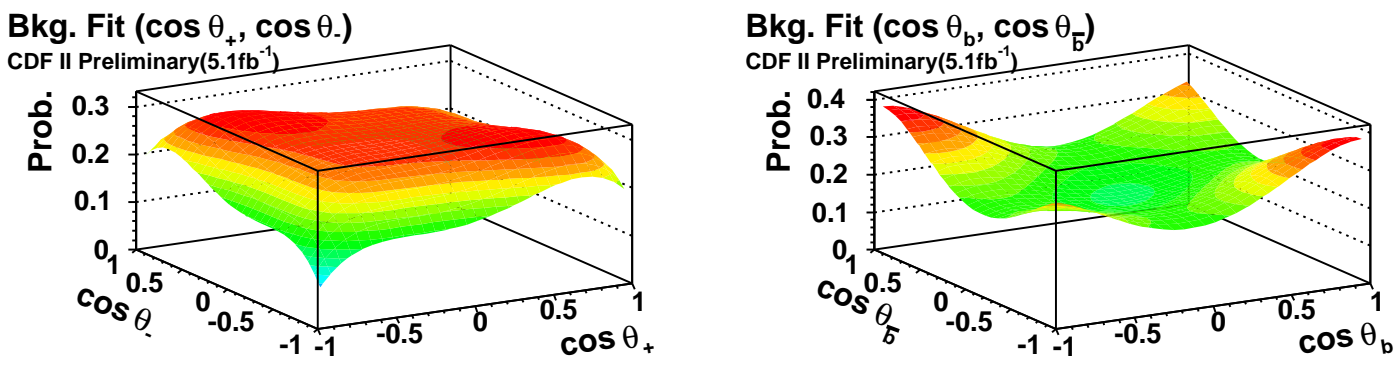

Figure 4.14: The distributions of reconstructed $\left(\cos \theta_{+}, \cos \theta_{-}\right)$(left) and $\left(\cos \theta_{b}, \cos \theta_{\bar{b}}\right)$ (right) of accumulated dilepton candidates from $W W / W Z / Z Z, Z \rightarrow \tau \tau$, $Z / \gamma^{*} \rightarrow e e, \mu \mu$, and fake samples. The distributions in the middle row indicate the magnitude of $1 \sigma$ uncertainty of each bin of the distributions in left column. The surfaces in the lower row are fit results of each distribution. 


\subsubsection{Crosscheck of the Signal and the Background shape}

We check the modeling of background amd kinematical reconstruction method by comparing distributions of observed candidates in $5.1 \mathrm{fb}^{-1}$ with prediction of signal and background.

We make the distribution of one dimentional distribution of reconstructed $\cos \theta$ of leptons and $\mathrm{b}$-jets, which are hardly depend on $\kappa$. The distributions are therefore suitable to use as a cross-check, keeping results blinded in this analysis.

Considering that $\cos \theta_{+}$and $\cos \theta_{b}$ are changed to $\cos \theta_{-}$ans $\cos \theta_{\bar{b}}$ under $\mathrm{CP}$ reversal, we compare the distribution of $\cos \theta_{+}$and $\cos \theta_{-}$, and the distribution of $\cos \theta_{b}$ and $\cos \theta_{\bar{b}}$ between data and prediction.

Figure 4.15 shows the resultant distributions. The cross indicates $5.1 \mathrm{fb}^{-1}$ data with statistical errors and red band indicates expected number of signal and background total events with $1 \sigma$ uncertainty.

We perform the Kolmogrov-Smirnov test to $\operatorname{compare} \cos \theta$ distribution of data to $\cos \theta$ distribution of prediction, for leptons and for bottoms, respectively [58]. The KolmogrovSmirnov probability are found to 0.71 for $\cos \theta$ distribution of leptons and 0.22 for $\cos \theta$ distribution of $b(\bar{b})$. The expected distributions and the distribution of data are in good agreement within the uncertainties for both of leptons and bottoms.
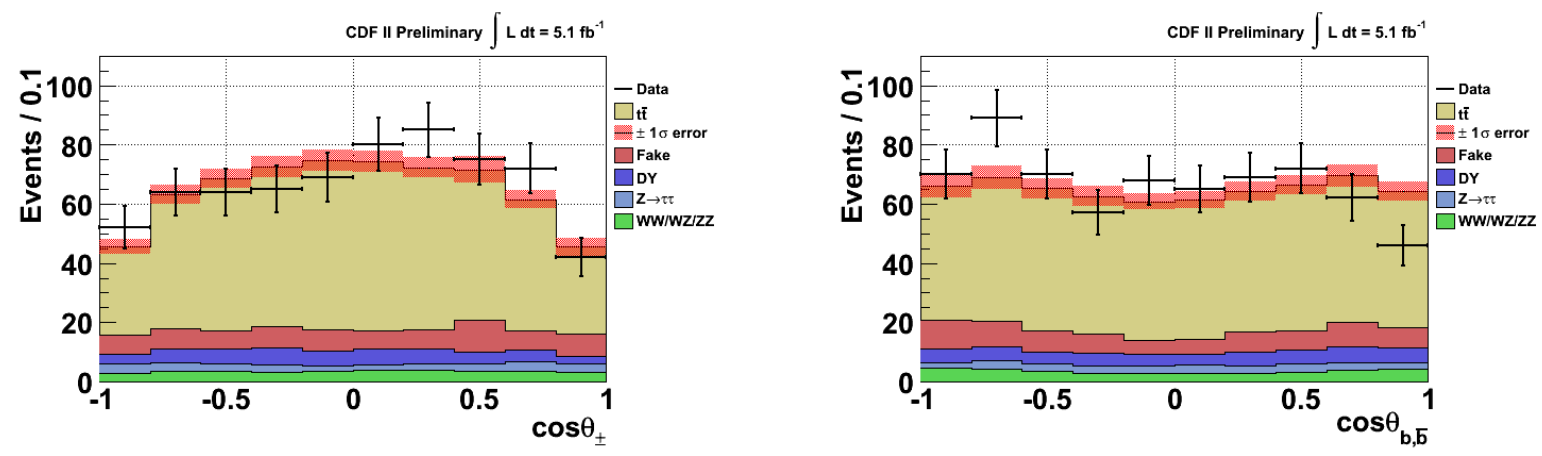

Figure 4.15: The distribution of $\cos \theta_{+}, \cos \theta_{-}$(left) and $\cos \theta_{b}, \cos \theta_{\bar{b}}$ (right). The cross indicates $5.1 \mathrm{fb}^{-1}$ data and red band indicates expected number of signal and background total events with $1 \sigma$ uncertainty. 


\subsection{Pseudo-experiments}

In this section, we describe a method to extract measured $\kappa$ from observed distributions of $\left(\cos \theta_{+}, \cos \theta_{-}\right)$and $\left(\cos \theta_{b}, \cos \theta_{\bar{b}}\right)$ first, and in order to verify the method and study sensitivity to $\kappa$ measurement, we perform pseudo-experiments.

In the previous section, we obtain signal and background templates, and using these templates we define the following likelihood as a function of assumed $\kappa$ :

$$
\mathcal{L}(\kappa)=\prod_{i} f^{\ell}\left(\cos \theta_{+}^{i}, \cos \theta_{-}^{i} ; \kappa\right) f^{b}\left(\cos \theta_{b}^{i}, \cos \theta_{\bar{b}}^{i} ; \kappa\right)
$$

where $i$ is index of candidates, and

$$
f^{\ell, b}(x, y ; \kappa) \equiv \frac{N_{\mathrm{exp}}^{\mathrm{sig}}}{N_{\exp }^{\mathrm{sig}}+N_{\exp }^{\mathrm{bkg}}} f_{\mathrm{sig}}^{\ell, b}(x, y ; \kappa)+\frac{N_{\mathrm{exp}}^{\mathrm{bkg}}}{N_{\exp }^{\mathrm{sig}}+N_{\exp }^{\mathrm{bkg}}} f_{\mathrm{bkg}}^{\ell, b}(x, y) .
$$

Here $N_{\text {exp }}^{\mathrm{sig}}$, and $N_{\exp }^{\mathrm{bkg}}$ represent the expected number of events for signal and background, respectively. $f_{\mathrm{sig}}^{\ell, b}(x, y ; \kappa)$, and $f_{\mathrm{bkg}}^{\ell, b}(x, y)$ represent template functions for $\left(\cos \theta_{+}, \cos \theta_{-}\right)$ and $\left(\cos \theta_{b}, \cos \theta_{\bar{b}}\right)$ of signal and background, respectively. We take $\kappa^{\text {meas }}$ as a measured $\kappa$, which gives maximum of the likelihood.

Note that this likelihood doesn't take correlations between $\left(\cos \theta_{+}, \cos \theta_{-}\right)$and $\left(\cos \theta_{b}, \cos \theta_{\bar{b}}\right)$ into account. In reality, we observe a correlation, though it is found to be very small. The likelihood might give a wrong estimate for statistical uncertainty due to this correlation. So, we do not use the likelihood to estimate statistical uncertainty of $\kappa^{\text {meas }}$, To exclude this inexpedience, we construct Feldman-Cousins confidence intervals from the distribution of $\kappa^{\text {meas }}$ itself by perform many pseudo-experiments as mentioned below. We exclude correlation by suppose prior probability with $\sigma\left(\kappa^{\text {meas }}\right)$ which contain correlation between $\left(\cos \theta_{+}, \cos \theta_{-}\right)$and $\left(\cos \theta_{b}, \cos \theta_{\bar{b}}\right)$.

For one pseudo-experiment, first we assume the following input values:

- true $\kappa$

- $N_{\text {obs }}$ : number of observed candidates

- $N_{\text {exp }}^{\text {sig }}$ : number of expected $t \bar{t}$ signal events

- $N_{\mathrm{exp}}^{\mathrm{bkg}}$ : number of expected background events

Then, we generate the following two random numbers:

- $N_{\mathrm{obs}}^{\text {sig }}$ : Poisson distributed with expected value of $N_{\exp }^{\mathrm{sig}}$

- $N_{\mathrm{obs}}^{\mathrm{bkg}}$ : Poisson distributed with expected value of $N_{\exp }^{\mathrm{bkg}}$

and repeat this generation until the condition

$$
N_{\mathrm{obs}}^{\mathrm{sig}}+N_{\mathrm{obs}}^{\mathrm{bkg}}=N_{\mathrm{obs}}
$$

is satisfied. 
Once we obtain $N_{\mathrm{obs}}^{\mathrm{sig}}$ and $N_{\mathrm{obs}}^{\mathrm{bkg}}$, we pick up $N_{\mathrm{obs}}^{\mathrm{sig}}$ set of $\left(\cos \theta_{+}, \cos \theta_{-}\right)$and $\left(\cos \theta_{b}, \cos \theta_{\bar{b}}\right)$ randomly from $t \bar{t}$ candidate event pool of signal Monte Carlo sample with probability proportional to $1+\kappa \cos \theta_{+}^{\text {hepg }} \cos \theta_{-}^{\text {hepg }}$. We next generate $N_{\text {obs }}^{\text {bkg }}$ random number set of $\left(\cos \theta_{+}, \cos \theta_{-}\right)$and $\left(\cos \theta_{b}, \cos \theta_{\bar{b}}\right)$ which are distributed by the background templates functions.

Then, we make the unbinned likelihood (Equation 4.8) to obtain $\kappa^{\text {meas }}$.
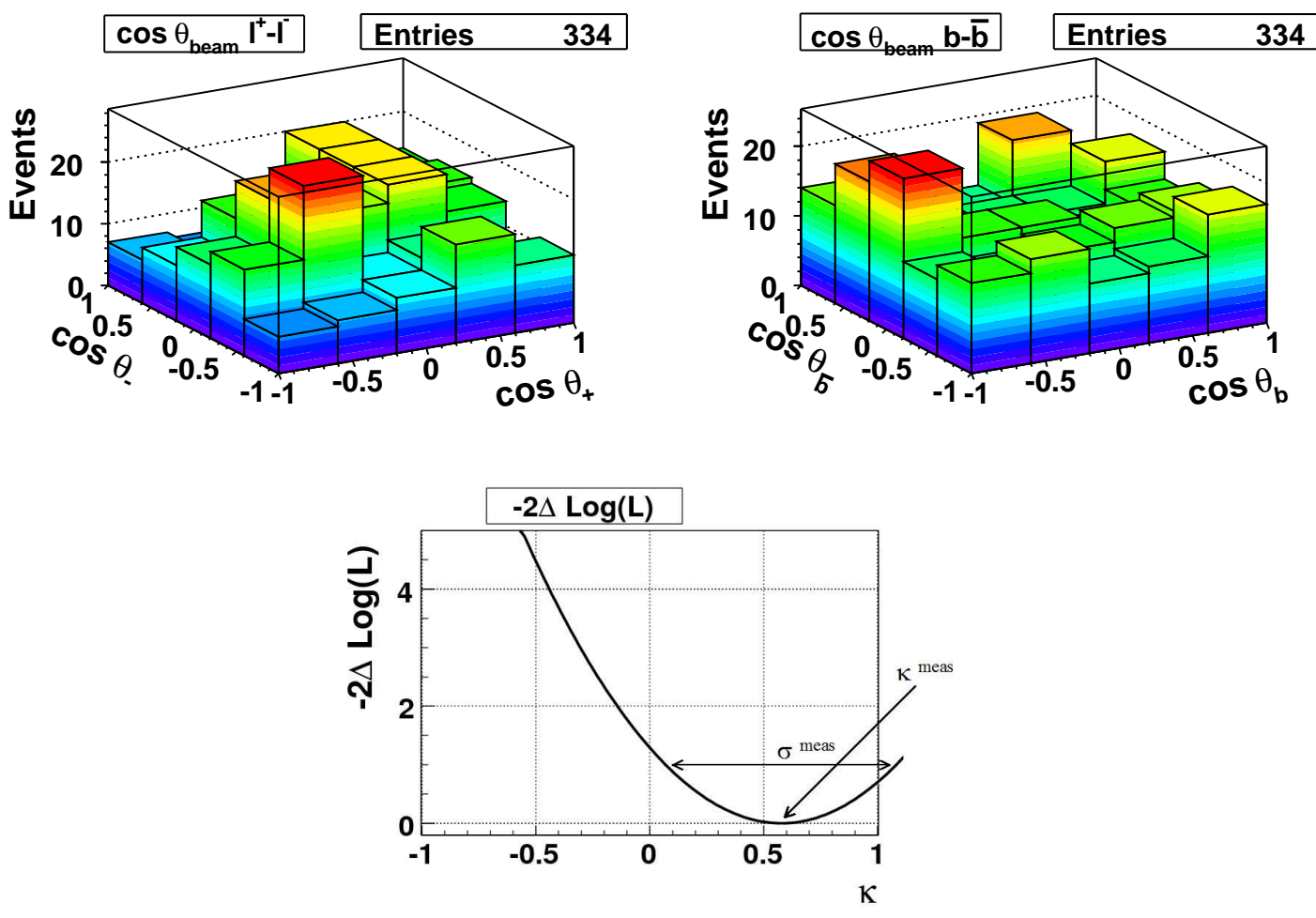

Figure 4.16: An example of pseudo data on the assumption of $\kappa^{\text {true }}=+1.0, N_{\text {obs }}=$ 334, $N_{\exp }^{\mathrm{sig}}=236.97$, and $N_{\exp }^{\mathrm{bkg}}=86.24$. Upper left: the distribution of reconstructed $\left(\cos \theta_{+}, \cos \theta_{-}\right)$. Upper right: the distribution of reconstructed $\left(\cos \theta_{b}, \cos \theta_{\bar{b}}\right)$. Lower: $-2 \Delta \log \mathcal{L}$ as a function of assumed $\kappa$. Measured $\kappa\left(\kappa^{\text {meas }}\right)$ is defined as the maximum point of the likelihood. By fitting $-2 \Delta \log \mathcal{L}$ to a quartic polynomial function, we allow unphysical region outside of $[-1,1]$ as $\kappa^{\text {meas }}$. Measured uncertainty of $\kappa^{\text {meas }}\left(\sigma^{\text {meas }}\right)$ is defined as the region of $-2 \Delta \log \mathcal{L}<1$.

Figure 4.16 illustrates an example of pseudo data on the assumption of $\kappa^{\text {true }}=+1.0$, $N_{\text {obs }}=334, N_{\text {exp }}^{\text {sig }}=236.97$, and $N_{\exp }^{\text {bkg }}=86.24$, and $-2 \Delta \log \mathcal{L}$ as a function of assumed $\kappa$. $\kappa^{\text {meas }}$ is defined as the maximum point of the likelihood. By fitting $-2 \Delta \log \mathcal{L}$ to a quartic polynomial function, we allow unphysical region outside of $[-1,1]$ as $\kappa^{\text {meas }}$ in order to construct Feldman-Cousins confidence intervals later. We also define $\sigma^{\text {meas }}$ by the region of $-2 \Delta \log \mathcal{L}<1$ as a measured uncertainty of $\kappa^{\text {meas }}$ to evaluate pull distribution later. 


\subsubsection{Check of the Method for $\kappa$ Measurement}

In order to check the method to extract $\kappa^{\text {meas }}$, signal templates, and procedure of the pseudo-experiment, we use $t \bar{t}$ Monte Carlo sample generated with Herwig. In this sample, standard model spin correlation is incorporated, and we found $\kappa^{\text {true }}=0.882 \pm 0.003$ by fitting distribution of $\left(\cos \theta_{+}^{\text {hepg }}, \cos \theta_{-}^{\text {hepg }}\right)$ in the whole dilepton events to a function of $1+\kappa \cos \theta_{+}^{\text {hepg }} \cos \theta_{-}^{\text {hepg }}$.
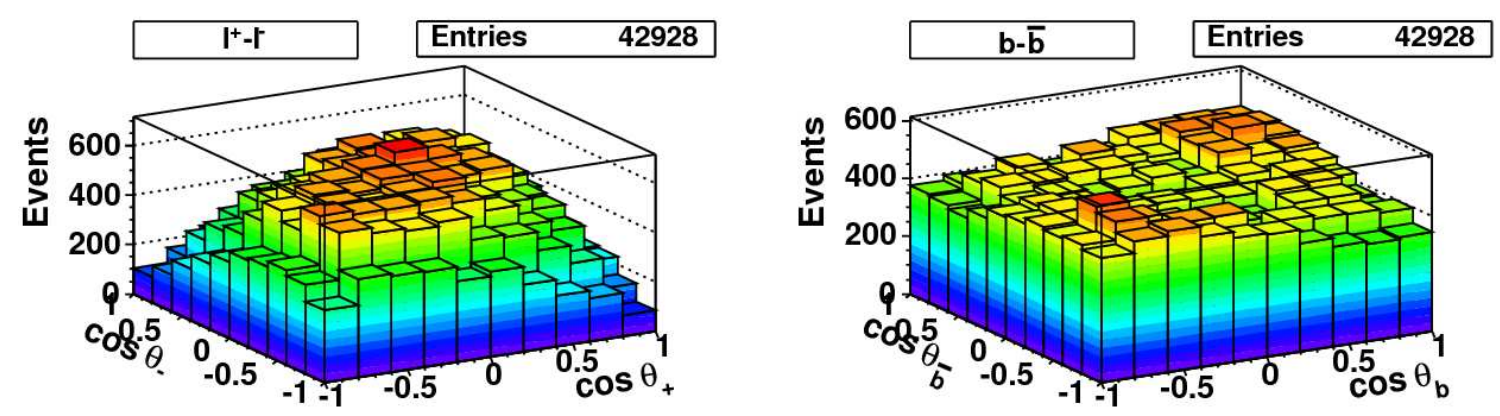

Figure 4.17: The distribution of reconstructed $\left(\cos \theta_{+}, \cos \theta_{-}\right)$(left) and $\left(\cos \theta_{b}, \cos \theta_{\bar{b}}\right)$ (right) of dilepton candidates in Monte Carlo sample which is made with Herwig event generator where $t \bar{t}$ spin correlations are included.

Figure 4.17 shows distributions of reconstructed $\left(\cos \theta_{+}, \cos \theta_{-}\right)$and $\left(\cos \theta_{b}, \cos \theta_{\bar{b}}\right)$ of dilepton candidates in herwig Monte Carlo sample in 10 by 10 bin histograms.

First we assume $N_{\text {obs }}=N_{\text {exp }}^{\text {sig }}=130$, and $N_{\text {exp }}^{\text {bkg }}=0$, which corresponds a scenario with ideal $b$-tag situation that has $55 \%$ event efficiency for $t \bar{t}$ event and perfect rejection for background. On the assumption, we perform 10,000 times pseudo-experiment by picking up $N_{\text {obs }}$ events from dilepton candidate pool in herwig Monte Carlo, and look at the distribution of $\kappa^{\text {meas }}$.

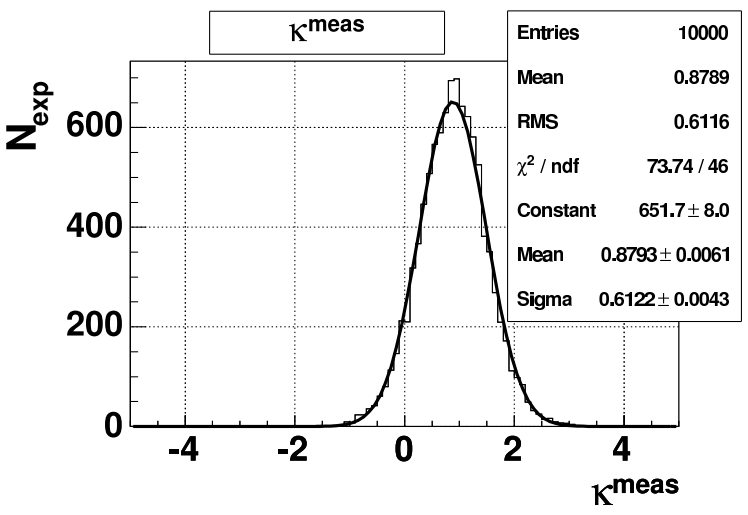

Figure 4.18: The distribution of $\kappa^{\text {meas }}$ of 10,000 pseudo-experiments using herwig Monte Carlo as signal sample pool on assumption of $N_{\text {obs }}=130, N_{\exp }^{\text {sig }}=130$, and $N_{\exp }^{\text {bkg }}=0$. The curve is the Gaussian fit of the distribution, and we find $\left\langle\kappa^{\text {meas }}\right\rangle=0.879 \pm 0.006$ and $\sigma\left(\kappa^{\text {meas }}\right)=0.612 \pm 0.004$. 
Figure 4.18 shows the resultant distribution. By the Gaussian fit of the distribution, mean of $\kappa^{\text {meas }}$ is found to be $\left\langle\kappa^{\text {meas }}\right\rangle=0.879 \pm 0.006$, that is consistent with $\kappa^{\text {true }}$, and uncertainty of $\kappa^{\text {meas }}$ is found to be $\sigma\left(\kappa^{\text {meas }}\right)=0.612 \pm 0.004$.

Next, we assume numbers of $5.1 \mathrm{fb}^{-1}$, i.e. $N_{\mathrm{obs}}=334, N_{\exp }^{\mathrm{sig}}=236.97$, and $N_{\exp }^{\mathrm{bkg}}=$ 86.24 .

On the assumption, we perform 10,000 times pseudo-experiment using herwig Monte Carlo signal pool for pseudo signal event and background template for pseudo background event.

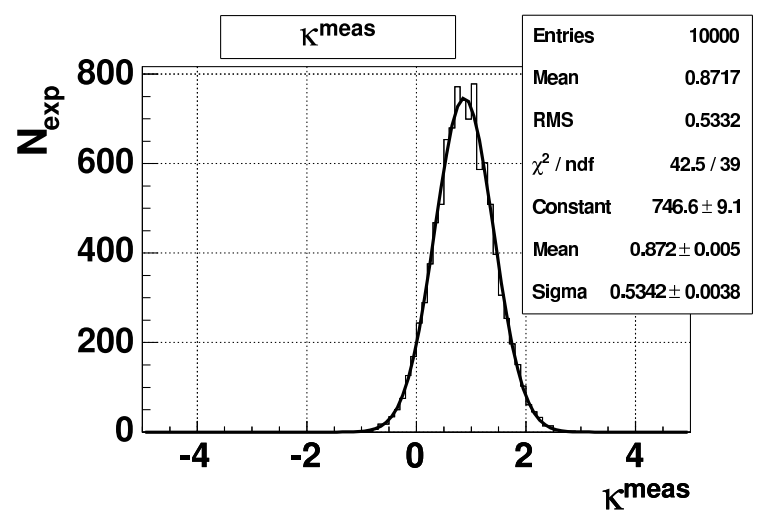

Figure 4.19: The distribution of $\kappa^{\text {meas }}$ of 10,000 pseudo-experiments using herwig Monte Carlo as signal sample pool on assumption of $N_{\text {obs }}=334, N_{\exp }^{\text {sig }}=236.97$, and $N_{\exp }^{\text {bkg }}=$ 86.24. The curve is the Gaussian fit of the distribution, and we find $\left\langle\kappa^{\text {meas }}\right\rangle=0.872 \pm$ 0.005 and $\sigma\left(\kappa^{\text {meas }}\right)=0.534 \pm 0.004$.

Figure 4.19 shows the resultant distribution. By the Gaussian fit of the distribution, mean of $\kappa^{\text {meas }}$ is found to be $\left\langle\kappa^{\text {meas }}\right\rangle=0.872 \pm 0.005$, that is consistent with $\kappa^{\text {true }}$ as well, and uncertainty of $\kappa^{\text {meas }}$ is found to be $\sigma\left(\kappa^{\text {meas }}\right)=0.534 \pm 0.004$, that is nearly equal to the case with ideal $b$-tag scenario above. 


\subsubsection{Pseudo Experiment Results}

Here in order to obtain $\left\langle\kappa^{\text {meas }}\right\rangle$ and $\sigma\left(\kappa^{\text {meas }}\right)$ as functions of $\kappa^{\text {true }}$, we perform 10,000 pseudo-experiments for each $\kappa^{\text {true }}$ ranging from -1 to +1 with 0.1 step, using the prescription described in the beginning of this section to cope with Pythia $t \bar{t}$ sample which doesn't have spin correlation.
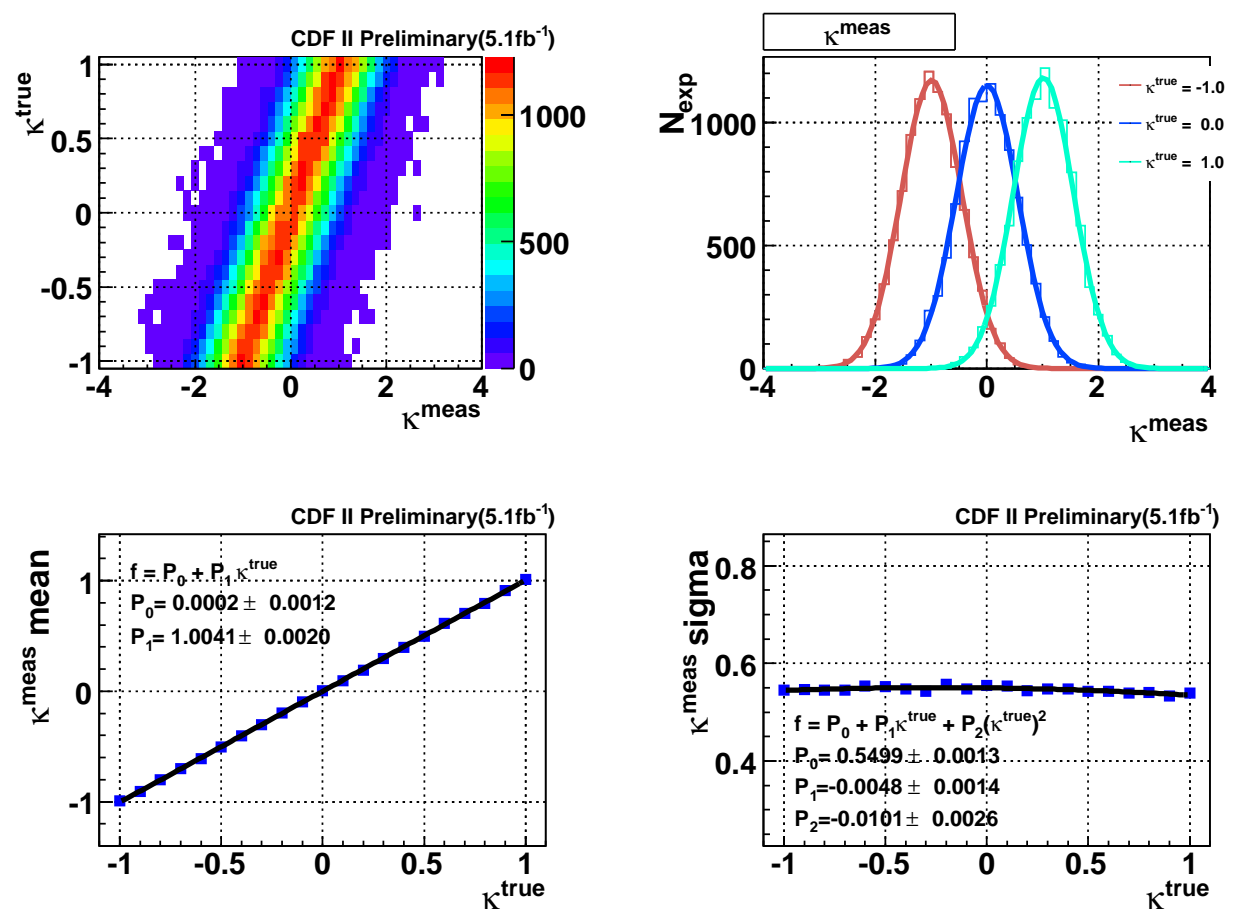

Figure 4.20: The result of 10,000 pseudo-experiments on the assumption of $\kappa^{\text {true }}=$ $-1.0 \sim+1.0, N_{\text {obs }}=334, N_{\exp }^{\text {sig }}=236.97$, and $N_{\exp }^{\text {bkg }}=86.24$. Upper left: The distribution of $\kappa^{\text {meas }}$ (horizontal axis) as a function of $\kappa^{\text {true }}$ (vertical axis). Upper right: The distribution of $\kappa^{\text {meas }}$ in case of $\kappa^{\text {true }}=-1.0, \kappa^{\text {true }}=0.0$, and $\kappa^{\text {true }}=+1.0$, and Gaussian fit curve, respectively. Lower left: $\left\langle\kappa^{\text {meas }}\right\rangle$ as a function of $\kappa^{\text {true }}$, and linear fit. Lower right: $\sigma\left(\kappa^{\text {meas }}\right)$ as a function of $\kappa^{\text {true }}$, and parabolic fit.

Figure 4.20 shows the result of 10,000 pseudo-experiments on the assumption of $\kappa^{\text {true }}=-1.0 \sim+1.0, N_{\text {obs }}=334, N_{\text {exp }}^{\text {sig }}=236.97$, and $N_{\exp }^{\text {bkg }}=86.24$. By Gaussian fit of a distribution of $\kappa^{\text {meas }}$ for each $\kappa^{\text {true }}$, we obtain $\left\langle\kappa^{\text {meas }}\right\rangle$ and $\sigma\left(\kappa^{\text {meas }}\right)$ for each $\kappa^{\text {true }}$. Finally, we obtain $\left\langle\kappa^{\text {meas }}\right\rangle$ as a linear function

$$
\begin{aligned}
\left\langle\kappa^{\text {meas }}\right\rangle & =P_{0}+P_{1} \kappa^{\text {true }} \\
P_{0} & =0.0002 \pm 0.0012 \\
P_{1} & =1.0041 \pm 0.0020
\end{aligned}
$$


and $\sigma\left(\kappa^{\text {meas }}\right)$ as a parabolic function

$$
\begin{aligned}
\sigma\left(\kappa^{\text {meas }}\right) & =P_{0}+P_{1} \kappa^{\text {true }}+P_{2}\left(\kappa^{\text {true }}\right)^{2} \\
P_{0} & =0.5499 \pm 0.0013 \\
P_{1} & =-0.0048 \pm 0.0014 \\
P_{2} & =-0.0101 \pm 0.0026
\end{aligned}
$$

by fitting of plots, respectively.

A small offset is seen in $\left\langle\kappa^{\text {meas }}\right\rangle$. This offset will be properly incorporated in the final result since we construct Feldman-Cousins confidence intervals with this $\left\langle\kappa^{\text {meas }}\right\rangle$ itself and $\sigma\left(\kappa^{\text {meas }}\right)$.
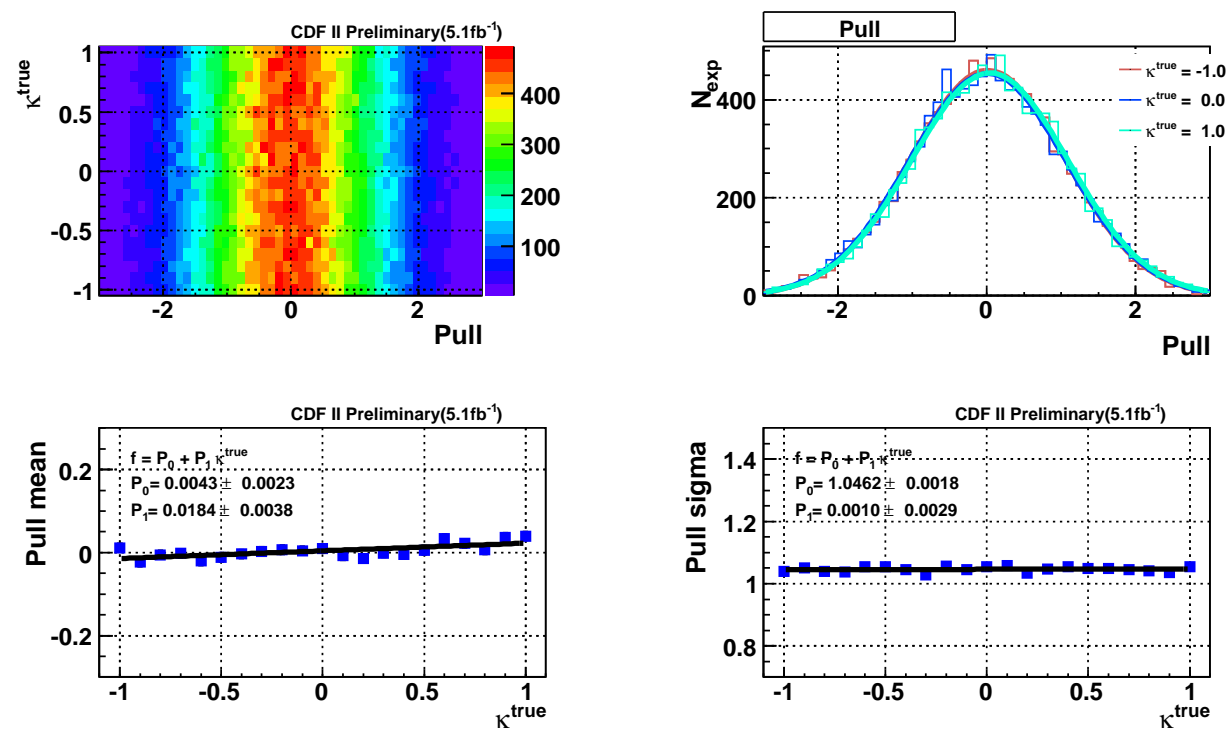

Figure 4.21: The pull distribution of 10,000 pseudo-experiments on the assumption of $\kappa^{\text {true }}=-1.0 \sim+1.0, N_{\text {obs }}=334, N_{\exp }^{\text {sig }}=236.97$, and $N_{\exp }^{\text {bkg }}=86.24$. Upper left: The pull $\left(\equiv\left(\kappa^{\text {meas }}-\kappa^{\text {true }}\right) / \sigma^{\text {meas }}\right)$ distribution as a function of $\kappa^{\text {true }}$. Upper right: The pull distribution in case of $\kappa^{\text {true }}=-1.0, \kappa^{\text {true }}=0.0$, and $\kappa^{\text {true }}=+1.0$, and Gaussian fit curve, respectively. Lower left: pull mean as a function of $\kappa^{\text {true }}$, and linear fit. Lower right: pull width as a function of $\kappa^{\text {true }}$, and linear fit.

Figure 4.21 shows the pull distribution of 10,000 pseudo-experiments for each $\kappa^{\text {true }}$. The pull is defined by

$$
\text { pull } \equiv\left(\kappa^{\text {meas }}-\kappa^{\text {true }}\right) / \sigma^{\text {meas }} .
$$

By fitting pull distribution for each $\kappa^{\text {true }}$ to a Gaussian curve, we obtain pull mean and pull sigma as functions of $\kappa^{\text {true }}$. We observe pull sigma is a little bit grater than unit. We found this is mainly caused since $\sigma^{\text {meas }}$ is estimated smaller due to correlation between the distributions of $\left(\cos \theta_{+}, \cos \theta_{-}\right)$and $\left(\cos \theta_{b}, \cos \theta_{\bar{b}}\right)$. We check this by extracting $\kappa^{\text {meas }}$ with only $\left(\cos \theta_{+}, \cos \theta_{-}\right)$and with only $\left(\cos \theta_{b}, \cos \theta_{\bar{b}}\right)$, separately. 


\subsubsection{Correlation between $\kappa^{\text {meas }}$ from leptons and $\kappa^{\text {meas }}$ from $b$-jets}

In order to check correlation between $\kappa^{\text {meas }}$ obtained only from $\left(\cos \theta_{+}, \cos \theta_{-}\right)$and $\kappa^{\text {meas }}$ obtained only $\left(\cos \theta_{b}, \cos \theta_{\bar{b}}\right)$, we performed 10,000 pseudo-experiments on the same condition in 4.6.2, but for likelihood instead of Equation 4.8 we just use the following $\mathcal{L}^{\ell}$ and $\mathcal{L}^{b}$, separately:

$$
\begin{aligned}
\mathcal{L}^{\ell}(\kappa) & =\prod_{i} f^{\ell}\left(\cos \theta_{+}^{i}, \cos \theta_{-}^{i} ; \kappa\right), \\
\mathcal{L}^{b}(\kappa) & =\prod_{i} f^{\ell}\left(\cos \theta_{b}^{i}, \cos \theta_{\bar{b}}^{i} ; \kappa\right)
\end{aligned}
$$

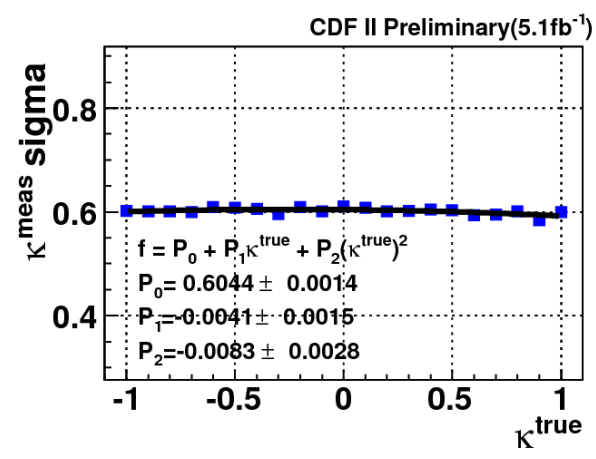

Figure 4.22: $\sigma\left(\kappa^{\text {meas }}\right)$ as a function of $\kappa^{\text {true }}$ for leptons only case.

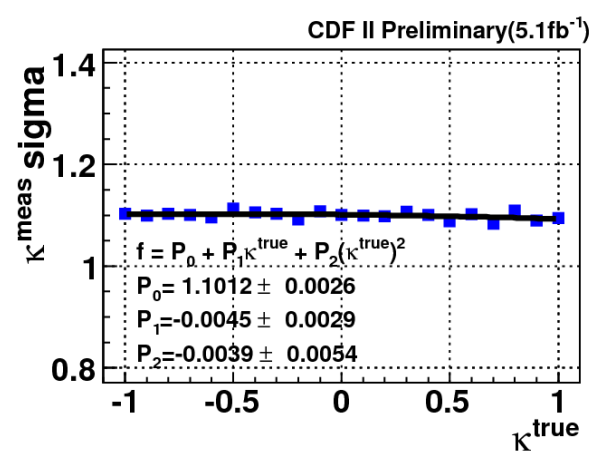

Figure 4.23: $\sigma\left(\kappa^{\text {meas }}\right)$ as a function of $\kappa^{\text {true }}$ for b-jets only case.

Fig 4.22 and 4.23 show the $\sigma\left(\kappa^{\text {meas }}\right)$ as a function of $\kappa^{\text {true }}$ for leptons only case and that for b-jets only case, separately.

In Section 4.6.2, $\sigma\left(\kappa^{\text {meas }}\right)$ is about 0.55 at $\kappa^{\text {true }}=0$.

Comparing this, $\sigma\left(\kappa^{\text {meas }}\right)$ of leptons only case is about 0.60 at $\kappa^{\text {true }}=0$ and $\sigma\left(\kappa^{\text {meas }}\right)$ of $b$-jets only case is about 1.10 at $\kappa^{\text {true }}=0$. A naive combination of statistical uncertain- 
ties of these results suppose they were independent is

$$
\frac{1}{\sqrt{\left(\frac{1}{0.60}\right)^{2}+\left(\frac{1}{1.10}\right)^{2}}}=0.53 .
$$

This value is close to 0.55 but a little bit smaller. Hence we conclude there is a small but some correlation between $\kappa^{\text {meas }}$ obtained only from $\left(\cos \theta_{+}, \cos \theta_{-}\right)$and $\kappa^{\text {meas }}$ obtained only from $\left(\cos \theta_{b}, \cos \theta_{\bar{b}}\right)$. And leptons $+b$-jets case in Section 4.6.2, 0.55, is improved by about $8 \%$ comparing with leptons only case. 


\subsubsection{Construction of Feldman-Cousins intervals}

Using $\left\langle\kappa^{\text {meas }}\right\rangle$ and $\sigma\left(\kappa^{\text {meas }}\right)$ obtained in Sec 4.6.2, we construction of Feldman-Cousins confidence interval [59] as a function of $\kappa^{\text {true }}$.

We first suppose $P\left(\kappa^{\text {meas }} \mid \kappa^{\text {true }}\right)$ as the probability that $\kappa^{\text {meas }}$ is observed at a given $\kappa^{\text {true }}$. We suppose the probability is given by normal distribution of $N\left(\left\langle\kappa^{\text {meas }}\right\rangle, \sigma\left(\kappa^{\text {meas }}\right)\right)$.

Then we set an interval $\left[\kappa_{1}^{\text {meas }}, \kappa_{2}^{\text {meas }}\right]$ so that the integral of the probability from $\kappa_{1}^{\text {meas }}$ to $\kappa_{2}^{\text {meas }}$ is a given confidence level, i.e.

$$
\int_{\kappa_{1}^{\text {meas }}}^{\kappa_{2}^{\text {meas }}} P\left(\kappa^{\text {meas }} \mid \kappa^{\text {true }}\right) d \kappa^{\text {meas }}=\text { C.L. , }
$$

using Feldman-Cousins's ordering principle. In the principle, we define $\kappa_{\text {best }}^{\text {true }}$ which gives maximum probability within physically allowed region of $\kappa^{\text {true }}$ when a $\kappa^{\text {meas }}$ is given, then require

$$
R\left(\kappa_{1}^{\text {meas }}\right)=R\left(\kappa_{2}^{\text {meas }}\right)
$$

where

$$
R\left(\kappa^{\text {meas }}\right) \equiv \frac{P\left(\kappa^{\text {meas }} \mid \kappa^{\text {true }}\right)}{P\left(\kappa^{\text {meas }} \mid \kappa_{\text {best }}^{\text {true }}\right)} .
$$

Figure 4.24 shows confidence intervals at $68 \%$ and $95 \%$ level, as a function of $\kappa^{\text {true }}$, respectively.

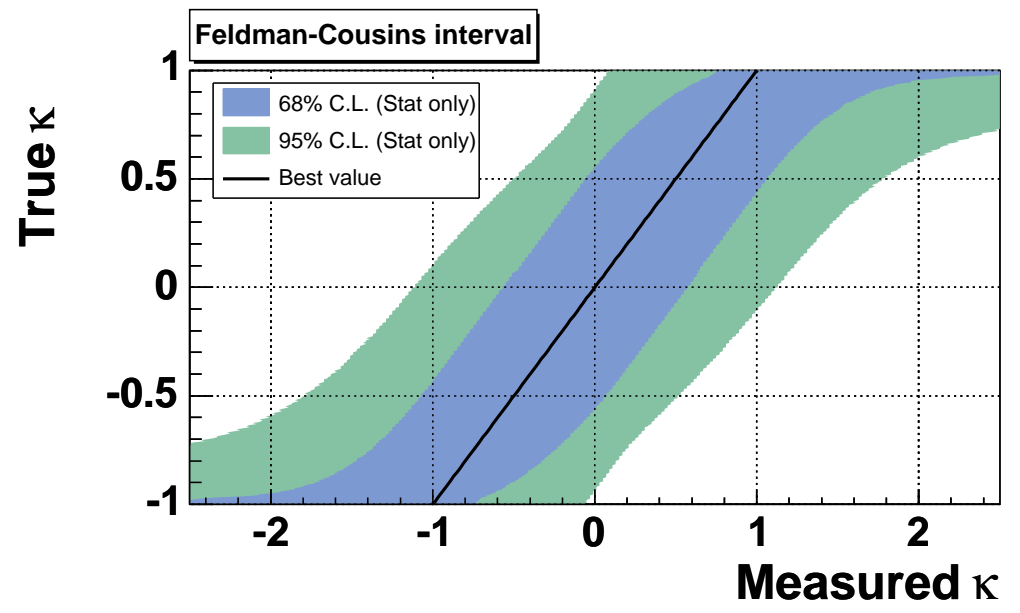

Figure 4.24: Confidence interval at $68 \%$ and $95 \%$ level based on Feldman-Cousins prescription as a function of $\kappa^{\text {true }}$, where the distribution of $\kappa^{\text {meas }}$ is assumed to be Gaussian with $\left\langle\kappa^{\text {meas }}\right\rangle$ and $\sigma\left(\kappa^{\text {meas }}\right)$ obtained from pseudo-experiments. Only statistical errors are considered here. 



\section{Chapter 5}

\section{Systematic Uncertainties}

We have performed systematics studies with respect to known sources. They are Statistical uncertainty of signal MC sample, Uncertainties in expected signal background numbers and background templates, jet energy, ISR/FSR, gg fraction, PDF, NLO and color reconnection. To estimate these systematics, we performed pseudo experiment.

\subsection{Statistical Uncertainty of Signal MC Sample}

In this analysis, the signal templates depend on Monte Carlo $t \bar{t}$ sample. The number of $t \bar{t}$ candidates in the sample is finite. Therefore, the signal templates themselves suffer statistical fluctuation.

To estimate the effect of signal MC statistics, we perform 10,000 pseudo experiments for each $\kappa^{\text {true }}$, where we have 100 signal only events and no background for each experiment. Then we obtain $\sigma\left(\kappa^{\text {meas }}\right)$ for 100 signal only case.

In the nominal $t \bar{t}$ signal sample we use, we have 47,898 candidates. Consequently, we obtain $\sigma\left(\kappa^{\text {meas }}\right)$ due to the statistical fluctuation of signal templates by re-scaling by the scale factor of $\sqrt{100 / 47898}$.

Figure 5.1 shows expected $\sigma\left(\kappa^{\text {meas }}\right)$ for the case of 47,898 signals.

We add this $\sigma\left(\kappa^{\text {meas }}\right)$ into systematic uncertainty in quadrature as systematics due to statistical uncertainty of signal Monte Carlo sample.

\subsection{Uncertainties in expected signal, background num- bers and background templates}

As described in Section 4.6, in a nominal pseudo experiment, we generate pseudo background events as random numbers of $\left(\cos \theta_{+}, \cos \theta_{-}\right)$and $\left(\cos \theta_{b}, \cos \theta_{\bar{b}}\right)$ which distribute the background templates defined in Section 4.5.3.

However, we have to consider uncertainties in the background templates and look at effects on measured $\kappa$. The possible uncertainties in the background templates come from

- Uncertainty of expected number of each background component 


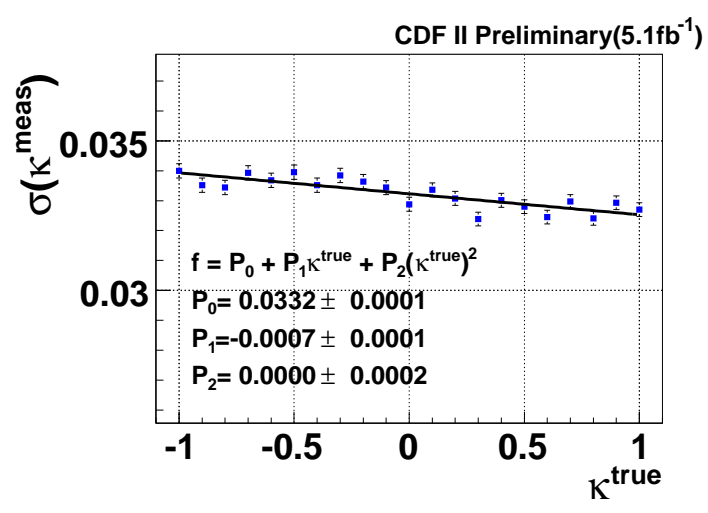

Figure 5.1: Expected $\sigma\left(\kappa^{\text {meas }}\right)$ for the case that all entries $(47,898)$ in signal MC is used. This is obtained by scaling $\sigma\left(\kappa^{\text {meas }}\right)$ of 100 signal only case by $\sqrt{100 / 47898}$.

- Statistical uncertainties of Monte Carlo and/or data samples which are used to define background templates.

To estimate these effects, we perform an alternative set of pseudo-experiments described below. In these pseudo-experiments, we also consider the uncertainty in expected number of signal events.

1. We use uncertainties of expected numbers of signal and each background component in table 4.1. Following these uncertainties, we generate following Gaussian randoms:

$$
\begin{aligned}
N_{\exp }^{\prime \text { sig }}= & \operatorname{Gaus}\left(N_{\exp }^{\text {sig }}, \delta N_{\exp }^{\text {sig }}\right) \\
N_{\exp }^{\prime W W}= & \operatorname{Gaus}\left(N_{\exp }^{W W}, \delta N_{\exp }^{W W}\right) \\
\cdots & \cdots \\
N_{\exp }^{\prime \text { fake }}= & \operatorname{Gaus}\left(N_{\exp }^{\text {fake }}, \delta N_{\exp }^{\text {fake }}\right),
\end{aligned}
$$

where $N^{\prime}$ indicates fluctuated expected number.

Then we take sum over all background components:

$$
N_{\exp }^{\prime \mathrm{bkg}}=\sum_{i} N_{\exp }^{\mathrm{bkg}_{i}}, \quad i=W W, W Z, \ldots, \text { fake }
$$

2. Using $N_{\exp }^{\prime \text { sig }}, N_{\exp }^{\prime \text { bkg }}$, and $N_{\text {obs }}$, we generate observed numbers as Poisson randoms:

$$
\begin{aligned}
N_{\mathrm{obs}}^{\mathrm{sig}} & =\operatorname{Poisson}\left(N_{\mathrm{exp}}^{\mathrm{\prime sig}}\right) \\
N_{\mathrm{obs}}^{\mathrm{bkg}} & =\operatorname{Poisson}\left(N_{\mathrm{exp}}^{\prime \text { bkg }}\right),
\end{aligned}
$$

where $N_{\text {obs }}^{\text {sig }}+N_{\text {obs }}^{\text {bkg }}=N_{\text {obs }}$. 
3. Next generate pseudo signal events by picking up $N_{\text {obs }}^{\text {sig }}$ events randomly from the signal candidate pool.

4. Generate pseudo background events: To estimate systematic uncertainties of background templates, we generate background events from fluctuated background template. There are five parameters in background template. Easy way to make fluctuated background templates is to fluctuate five parameters by normal distribution. However, five parameters have correlations therebetween. Therefore, we have to fluctuate five parameters considering these correlations. The method is as follows.

- First, we fluctuate fit parameters $\vec{C}=\left(C_{1}, \cdots, C_{5}\right)^{T}$ with consideration of correlation between each fit parameters. $\vec{C}^{\prime}=\left(C_{1}^{\prime}, \ldots, C_{5}^{\prime}\right)^{T}$ (fluctuated $\vec{C}$ ) is given by $\vec{C}^{\prime}=\vec{C}+L \vec{G}$, where $\vec{G}$ is vector of independent normalized Gaussian $(0,1)$ and $L$ is a matrix from Cholesky factorization [60], which satisfies $\Sigma=L L^{T}$, where $\Sigma$ is the covariance matrix of the fit parameters.

- Next, generate background template using $C_{i}^{\prime}(i=1, \cdots, 5)$.

- Then generate $N_{\mathrm{obs}}^{\mathrm{bkg}}$ random number sets of $\left(\cos \theta_{+}, \cos \theta_{-}\right)$and $\left(\cos \theta_{b}, \cos \theta_{\bar{b}}\right)$ which are distributed by the fluctuated background template functions.

5. Calculate unbinned likelihood for pseudo events of signal+background as a function of $\kappa$ on the assumption of nominal $N_{\exp }^{\mathrm{sig}}$ and $N_{\exp }^{\mathrm{bkg}}$, since we never know $N_{\text {exp }}^{\prime \text { sig }}$ nor $N_{\exp }^{\prime \text { bkg }}$. Then obtain $\kappa^{\text {meas }}$.

6. Make the distribution of $\kappa^{\text {meas }}$ by performing many pseudo-experiments $(10,000$ for each $\left.\kappa^{\text {true }}\right)$, then obtain $\left\langle\kappa^{\text {meas }}\right\rangle^{\prime}$, and $\sigma\left(\kappa^{\text {meas }}\right)^{\prime}$ as a mean and a standard deviation of each distribution.

7. Finally we calculate a shift in the mean of $\kappa^{\text {meas }}$ from the nominal and an increase of the standard deviation of $\kappa^{\text {meas }}$, i.e.

$$
\begin{aligned}
\Delta \kappa^{\text {meas }} & =\left\langle\kappa^{\text {meas }}\right\rangle^{\prime}-\left\langle\kappa^{\text {meas }}\right\rangle \\
\sigma^{\prime} & =\sqrt{\sigma\left(\kappa^{\text {meas }}\right)^{\prime 2}-\sigma\left(\kappa^{\text {meas }}\right)^{2}}
\end{aligned}
$$

We suppose the $\Delta \kappa^{\text {meas }}$ represents the effect from uncertainty of background template and $\sigma^{\prime}$ represents the effect from uncertainties of $N_{\exp }^{\mathrm{sig}}$ and $N_{\exp }^{\mathrm{bkg}_{i}}$, as well as statistical fluctuation in background distribution.

Figure 5.2 shows resultant $\Delta \kappa^{\text {meas }}$ and $\sigma^{\prime}$. We include both of two in quadrature as a systematic uncertainty from uncertainties in the expected signal, background numbers and background templates.

\subsection{Jet Energy Scale Uncertainty}

Jet energy uncertainty also affects dilepton acceptance as well as kinematical distributions. To estimate this effect, we perform pseudo experiments $(10,000$ experiments for each $\kappa$ ) where jet energy scale (JES) is shifted by $1 \sigma$ uncertainty as well as shifted by 


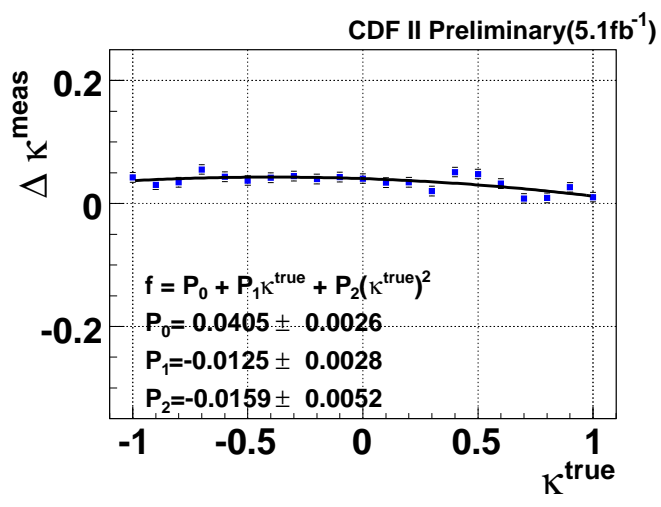

(a) shift of $\left\langle\kappa^{\text {meas }}\right\rangle$

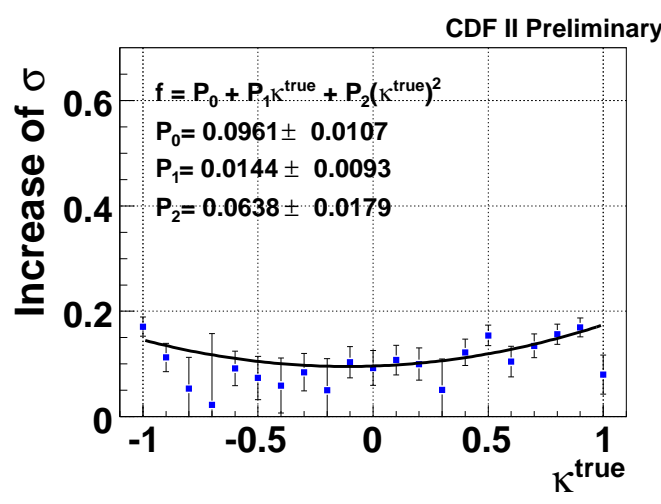

(b) increase of $\sigma\left(\kappa^{\text {meas }}\right)$

Figure 5.2: (a) Shift of $\left\langle\kappa^{\text {meas }}\right\rangle$ and (b) increase of $\sigma\left(\kappa^{\text {meas }}\right)$ from nominal pseudoexperiment case when we take fluctuations of uncertainties of signal, background numbers and background template function into account in the pseudo-experiment.

$-1 \sigma$. We also take shifts in $t \bar{t}$ acceptance into account. $N_{\exp }^{\text {sig }}$ is shifted by $+3.4 \%$ for JES $+1 \sigma$ shift, and by $-3.5 \%$ for JES $-1 \sigma$ shift.

Figure 5.3 shows shift of $\left\langle\kappa^{\text {meas }}\right\rangle$ from nominal pseudo-experiment case for jet energy scale $+1 \sigma$ case and $-1 \sigma$ case.

We look at shifts of $\left\langle\kappa^{\text {meas }}\right\rangle$ for two cases are in opposite direction. Therefore we take half difference as systematics from JES uncertainty, and add this into systematic uncertainty in quadrature.

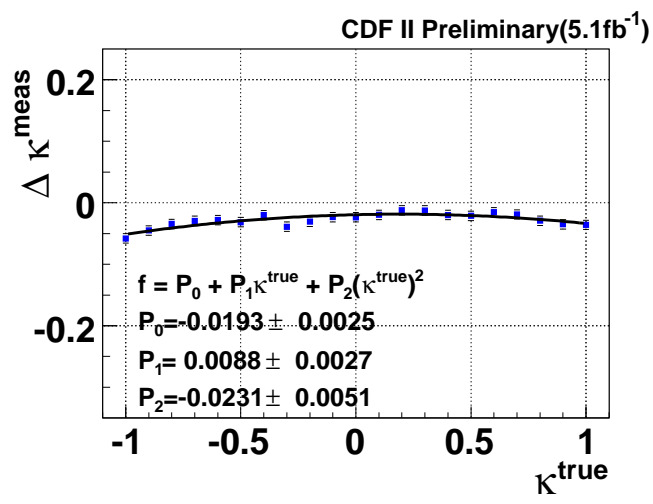

(a) JES $+1 \sigma$

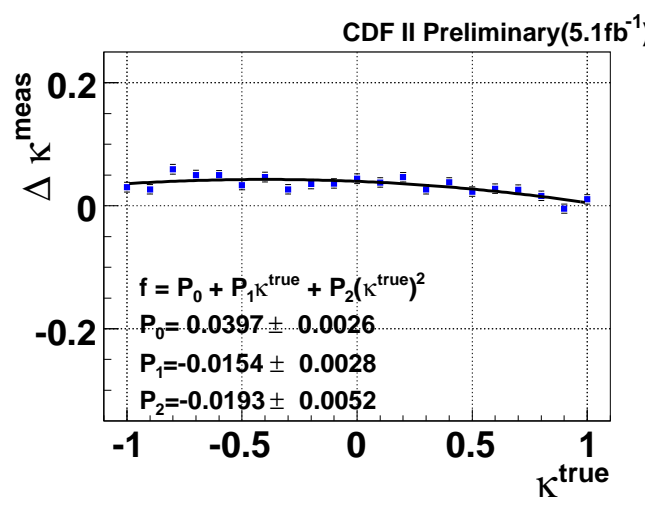

(b) JES $-1 \sigma$

Figure 5.3: Shift of $\left\langle\kappa^{\text {meas }}\right\rangle$ from nominal pseudo-experiment case when jet energy scale is (a) increased by $1 \sigma$ and (b) decreased by $1 \sigma$. 


\subsection{Initial and Final State Radiation}

Extra jets originating from the incoming partons and/or outgoing partons are called initial and final state radiations(ISR and FSR). Figure 5.4 shows $t \bar{t}$ diagram with ISR and FSR.

Differences due to QCD ISR and FSR are estimated using specially generated Pythia samples in which the QCD parameters for the parton shower evolution are varied based on the studies of the CDF Drell-Yan data and recommendations from the authors of PYTHIA.

ISR and FSR systematic uncertainties are estimated by changing the parameters related to ISR and FSR from their default values to half and double.

We look at shifts of $\left\langle\kappa^{\text {meas }}\right\rangle$ for two cases are in opposite direction. Figure 5.5 shows shift of $\left\langle\kappa^{\text {meas }}\right\rangle$ from nominal pseudo-experiment case for more ISR/FSR case and less ISR/FSR case. Therefore we take half difference as systematics from ISR/FSR uncertainty, and add this into systematic uncertainty in quadrature.

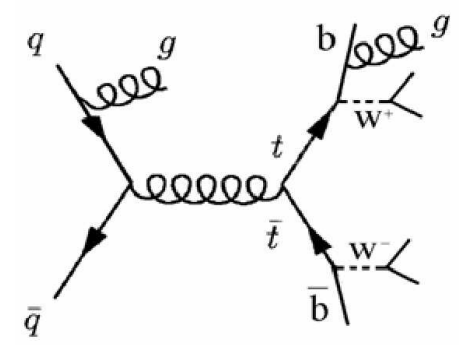

Figure 5.4: Diagram of $t \bar{t}$ production and decay with ISR and FSR.

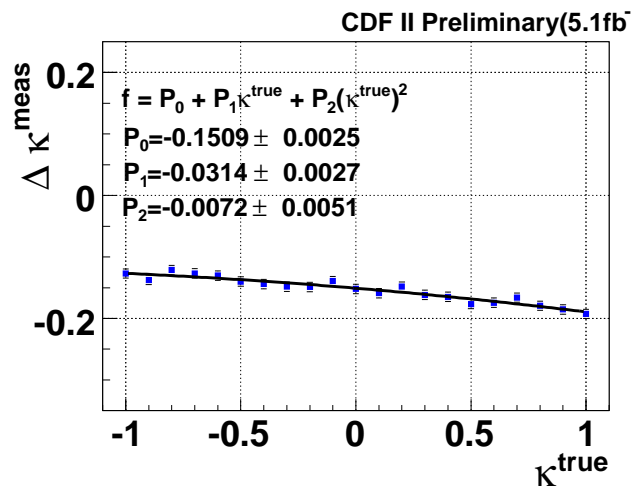

(a) ISR/FSR more

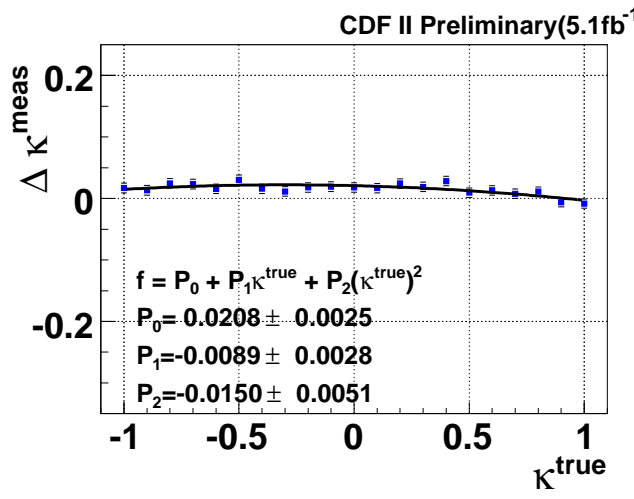

(b) ISR/FSR less

Figure 5.5: Shift of $\left\langle\kappa^{\text {meas }}\right\rangle$ from nominal pseudo-experiment case when (a) ISR/FSR more sample is used as signal pool in the pseudo-experiment and (b) ISR/FSR less sample is used. 


\subsection{Uncertainty of $g g$ fraction}

The nominal signal sample we use in this analysis is found to have about $6 \%$ of $t \bar{t}$ production via gluon gluon fusion process. However, this fraction is expected to be much bigger, at the level of around 15\%, in case of NLO calculation [61] [18].

In the full kinematical reconstruction method described in Section 4.4, the probability density functions of $p_{z}^{t \bar{t}}, p_{T}^{t \bar{t}}$, and $M_{t \bar{t}}$ used in Eqn. 4.2 depend on $t \bar{t}$ Monte Carlo which has only $6 \% g g$ fusion process. Due to difference of initial partons, we expect difference of the probability density functions between $q \bar{q}$ and $g g$ processes.

To estimate how much shift we observe in measured $\kappa$ if we increase the fraction of $g g$ fusion process, we perform pseudo-experiments (10,000 experiments for each $\kappa)$, where $15 \%$ is assumed for $g g$ fusion. We realize this by adopting an event by change of $(1-0.15) /(1-0.06)$ if the event originates from $q \bar{q}$ process when we pick up the event from the signal pool.

Figure 5.6 shows shift of $\left\langle\kappa^{\text {meas }}\right\rangle$ from nominal pseudo-experiment case. We add this shift into systematic uncertainty in quadrature, though it is found to be almost negligibly small effect.

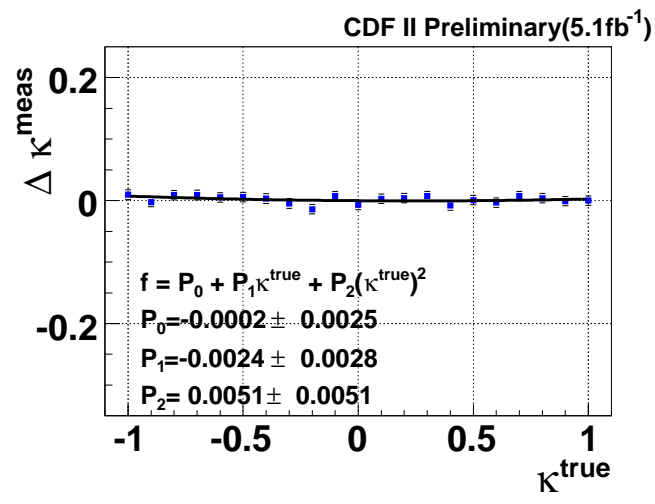

Figure 5.6: Shift of $\left\langle\kappa^{\text {meas }}\right\rangle$ from nominal pseudo-experiment case $\left(\mathcal{F}_{g g} \sim 6 \%\right)$ when $\mathcal{F}_{g g}=15 \%$ is assumed in the pseudo-experiment.

\subsection{Parton Distribution Functions}

For nominal signal sample, CTEQ5L parton distribution function (PDF) is supposed. To evaluate the systematics due to PDF uncertainties, we perform pseudo-experiments $(10,000$ experiments for each $\kappa)$ on the assumption of different PDF sets, such as MRST72, MRST75, CTEQ6L, CTEQ6L1 and CTEQ6M, and look how much shifts of measured $\kappa$ are caused.

To realize signal events with different PDF sets, we adopt the weighted MC method.In this method, we reweigh each event of a signal MC sample we already have, instead of generating a different set of MC events with each different PDF set. We calculate the 
relative probability of the event on the assumption of a different PDF set, based on the parton momentum fractions $x_{1}, x_{2}, Q^{2}$ and initial partons in the event. Then we pick up an event from the signal event pool according to new probability of an event in the pseudo-experiments.

To look at the effect of difference between MRST72 $\left(\Lambda_{Q C D}=228 \mathrm{MeV}\right)$ and CTEQ5L $\left(\Lambda_{Q C D}=300 \mathrm{MeV}\right)$ PDF set, we compare between $\left\langle\kappa^{\text {meas }}\right\rangle$ with MRST72 and one with CTEQ5L.

And next, to look at the effect of different $\alpha_{s}$ value, we compare between MRST72 and CTEQ75 cases.

Then, to look at the effect of uncertainties on the fitting parameters of CTEQ6M PDF set, we compare between CTEQ6M and CTEQ6M $n \pm$, where CTEQ6M $n \pm$ means CTEQ6M with $n$th of 20 orthogonal parameters are changed by $\pm 90 \%$. We sum up positive shifts and negative shifts asymmetrically in quadrature.

Finally, we take the larger effect either of the 20 eigenvectors uncertainty or the difference between MRST72 and CTEQ5L PDF sets, and add effect of different $\alpha_{s}$ in quadrature.

Figure 5.7 show resulting shifts of measured $\kappa$. Shift of $\left\langle\kappa^{\text {meas }}\right\rangle$ for MRST72 to CTEQ5L, difference of $\left\langle\kappa^{\text {meas }}\right\rangle$ between MRST72 and MRST75, and shifts of $\left\langle\kappa^{\text {meas }}\right\rangle$ by uncertainties of 20 eigenvectors in CTEQ6M PDF set are shown in the figure. And total uncertainties toward positive and negative direction are also shown.

We add them into into systematic uncertainty in quadrature as systematics from PDF uncertainty.

\subsection{Systematics from LO versus NLO}

In this analysis, we assume standard model $t \bar{t}$ production and top (anti-top) decays except for correlation coefficient between top and anti-top at the decays. This analysis relys on Monte Carlo with LO calculations for $t \bar{t}$ production kinematics such as $p_{z}^{t \bar{t}}, p_{T}^{t \bar{t}}$, and $M_{t \bar{t}}$. These might be changed at NLO calculations. Also there might be QCD interaction between final state products at NLO.

We examine the effect of NLO using $t \bar{t}$ Monte Carlo with an event generator at NLO(MC@NLO [62] [63]).

We perform pseudo experiments $(10,000$ experiments for each $\kappa)$, using $t \bar{t}$ Monte Calro generated from MC@NLO event generator with CTEQ5M and MRST02 for PDF, respectively.

Note that there is no spin correlation in these samples, therefore we treat $\kappa$ on these samples with the same prescription with nominal Pythia $t \bar{t}$ sample. Also note that about $11 \%$ of events are generated with negative weight in MC@NLO.

To cope with negative weight events, We pick up a positive weight event to cancel out negative event, if we pick up a negative weight event when generate performing pseudo experiment.

Figure 5.9 shows shift of $\left\langle\kappa^{\text {meas }}\right\rangle$ from nominal pseudo-experiment case for MC@ NLO with CTEQ5M and MRST02 cases, respectively. 

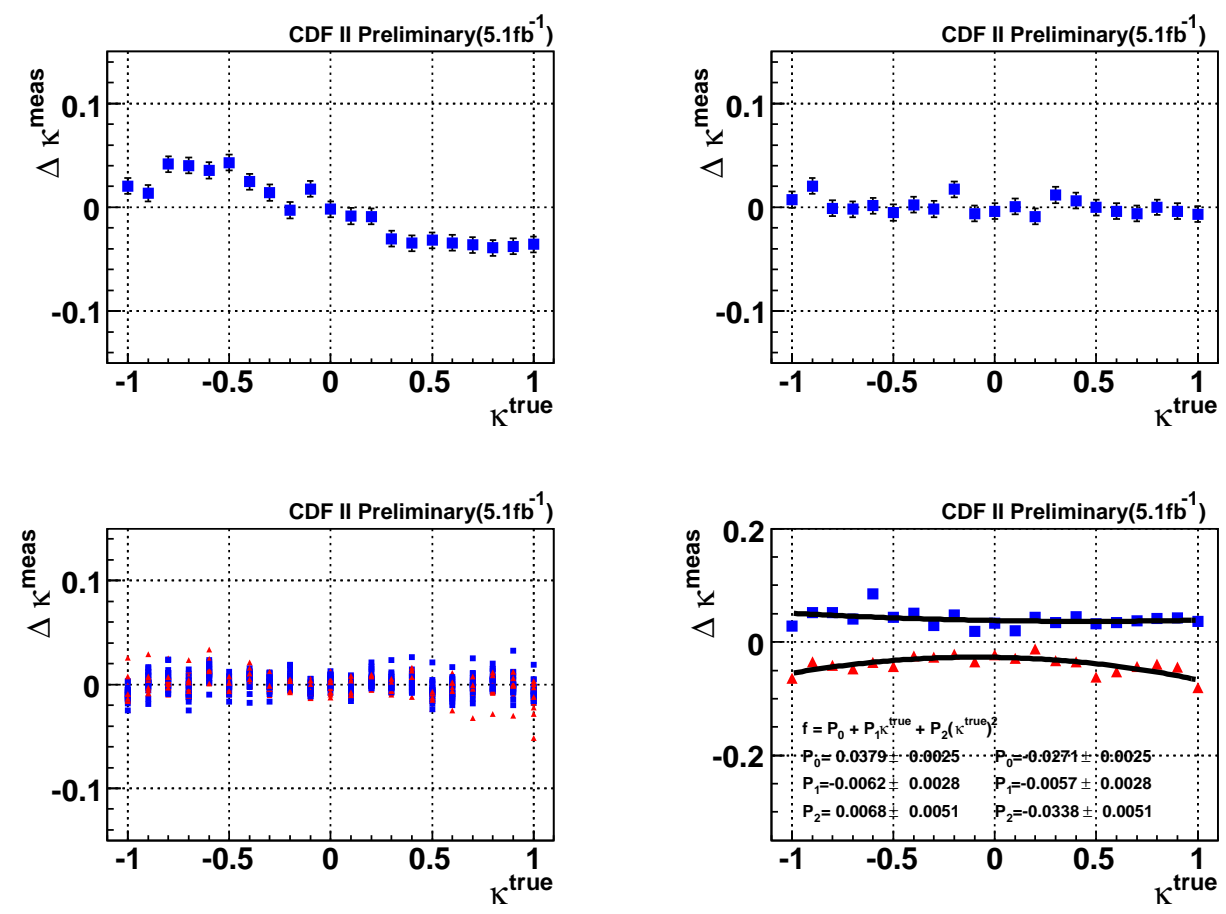

Figure 5.7: Shift of $\left\langle\kappa^{\text {meas }}\right\rangle$ for P.E. when MRST72 is assumed for PDF comparing with nominal P.E. with CTEQ5L (Upper left), difference of $\left\langle\kappa^{\text {meas }}\right\rangle$ between P.E. with MRST72 and MRST75 (Upper right), shifts of $\left\langle\kappa^{\text {meas }}\right\rangle$ for CTEQ6M where each parameter of 20 eigenvectors is shifted by $\pm 90 \%$ comparing with P.E. with nominal CTEQ6M (Lower left), and total uncertainties toward positive and negative direction of $\left\langle\kappa^{\text {meas }}\right\rangle$ from three sources of PDF uncertainties above (Lower right).

We take larger shift as systematics from NLO effect, and add this into systematic uncertainty in quadrature.

\subsection{Color Reconnection}

It has been suggested that color reconnection effects [64] could cause a bias in $\kappa$ measurement. To estimate this effect, we perform pseudo-experiments $(10,000$ experiments for each $\kappa$ ), using Pythia tune A [65] and Pythia tune $A_{C R}$ [65] as signal Monte Carlo samples, respectively. Pythia tune A is very similar to the tune for CDF nominal, Pythia tune $\mathrm{A}_{\mathrm{CR}}$ includes color reconnection effect into the tune $\mathrm{A}$. We compare between $\left\langle\kappa^{\text {meas }}\right\rangle$ with Pythia tune $A$ and one with Pythia tune $A_{C R}$.

Figure 5.10 shows difference of $\left\langle\kappa^{\text {meas }}\right\rangle$ between with and without color reconnection effect. We add this shift into systematic uncertainty in quadrature as systematics due to color reconnection effect. 


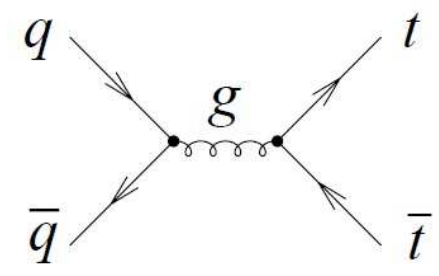

(a) Leading order

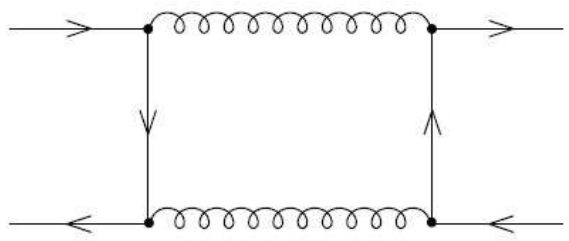

(b) Next to leading order

Figure 5.8: Feynman diagrams of $t \bar{t}$ production for LO and NLO.

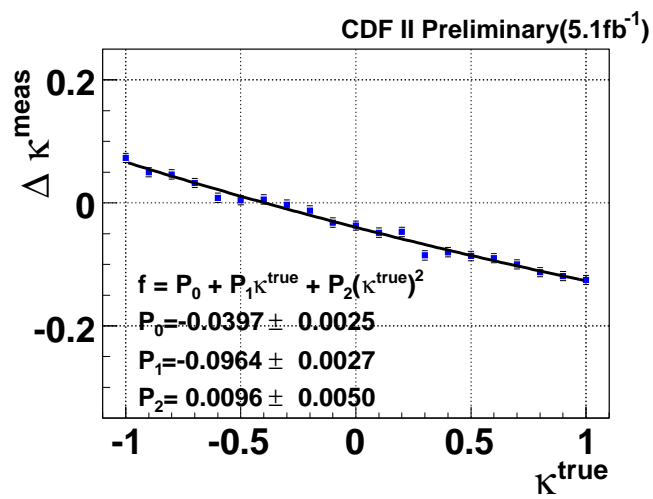

(a)

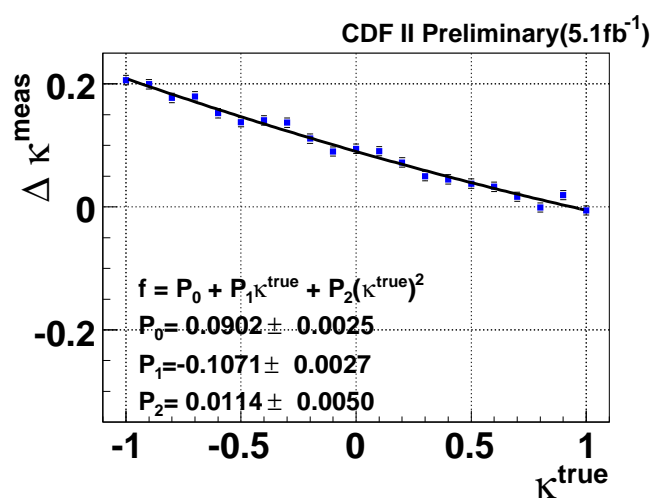

(b)

Figure 5.9: (a) Shift of $\left\langle\kappa^{\text {meas }}\right\rangle$ from nominal P.E. case when MC@NLO event generator with CTEQ5M as PDF is used. (b) Shift of $\left\langle\kappa^{\text {meas }}\right\rangle$ from nominal P.E. case when MC@NLO event generator with MRST02 as PDF is used.

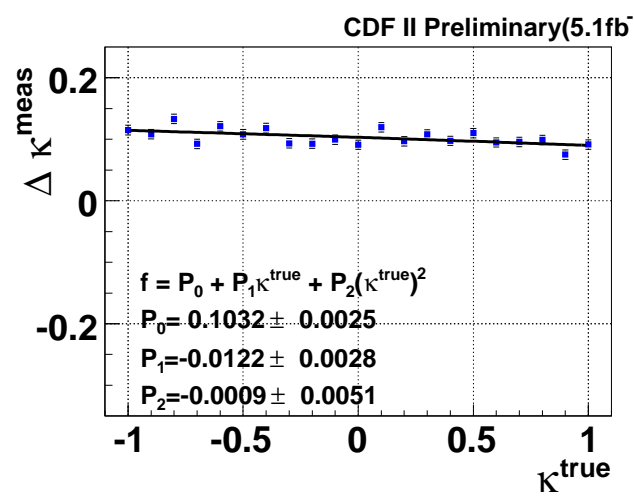

Figure 5.10: Difference of $\left\langle\kappa^{\text {meas }}\right\rangle$ with tune $\mathrm{A}$ and one with tune $\mathrm{A}_{C R}$. 


\subsection{Total Uncertainty and Feldman-Cousins Intervals}

We summarize here all possible systematics described above. And by adding this into statistical uncertainty in quadrature, we obtain total uncertainty including statistic and systematic effects to construct confidence belt as a function of $\kappa$. Table 5.1 shows summary of systematic uncertainty. Figure 5.9 shows total systematic uncertainty in $\left\langle\kappa^{\text {meas }}\right\rangle$ from all sources we discussed. We evaluate systematic uncertainties toward positive and negative direction separately. The figure 5.9 shows quadratic sum of statistical uncertainty and systematical uncertainty.

\begin{tabular}{c|c}
\hline \hline Source & Systematic uncertainty \\
\hline Statistical uncertainty of signal MC sample & $0.033 \sim 0.034$ \\
Expected signal, background numbers and background templates & $0.104 \sim 0.175$ \\
JES & $0.019 \sim 0.044$ \\
ISR/FSR & $0.071 \sim 0.093$ \\
gg fraction & $0.000 \sim 0.007$ \\
PDF & $0.037 \sim 0.051$ (toward positive) \\
NLO calculation & $0.027 \sim 0.067$ (toward negative) \\
Color reconnection & $0.068 \sim 0.209$ \\
Total systematic uncertainty & $0.115 \sim 0.092$ \\
\hline \hline
\end{tabular}

Table 5.1: The summary table of systematic uncertainty.

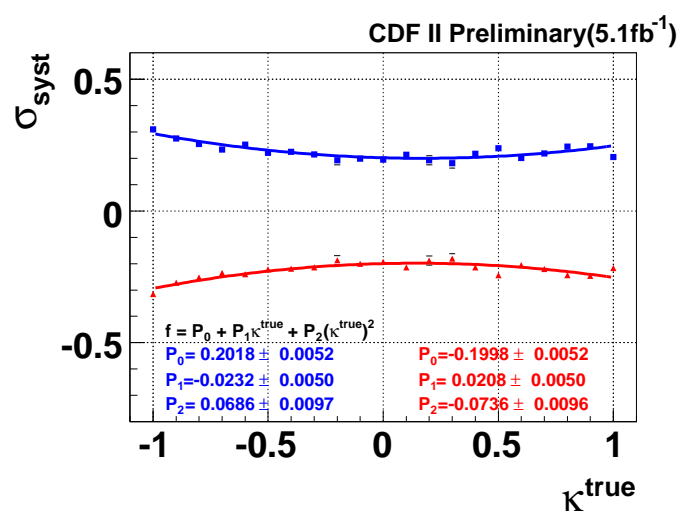

Figure 5.11: Total systematic uncertainty on $\kappa^{\text {meas }}$ from all sources we consider. Uncertainties toward positive(blue) and negative(red) direction are shown separately.

Figure 5.13 shows confidence interval at $68 \%$ and $95 \%$ level based on FeldmanCousins prescription as a function of $\kappa^{\text {true }}$, where the distribution of $\kappa^{\text {meas }}$ is assumed to be Gaussian with convolution of statistical and systematic errors.

Once we obtain a measured $\kappa\left(\kappa^{\text {meas }}\right)$ from beam data, we can set a confidence interval on true $\kappa$ from this plot. 


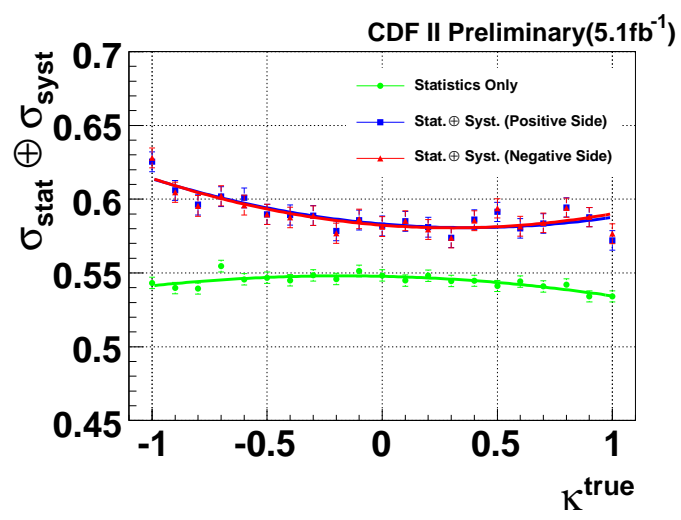

Figure 5.12: Statistical uncertainty (green) and statistical $\oplus$ systematical uncertainties (blue and red) on $\kappa^{\text {meas }}$ are shown separately.

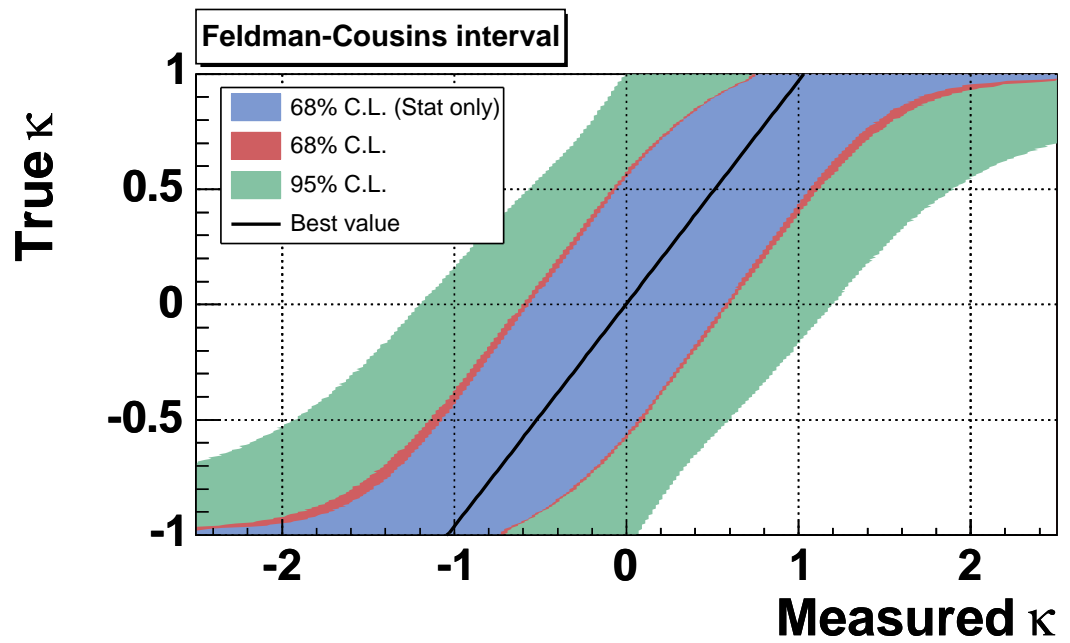

Figure 5.13: Confidence interval at $68 \%$ and $95 \%$ level based on Feldman-Cousins prescription as a function of $\kappa^{\text {true }}$, where the distribution of $\kappa^{\text {meas }}$ is assumed to be Gaussian with convolution of statistical error and systematic error. Also Feldman-Cousins confidence interval with only statistical error considered is shown in the same figure. 



\section{Chapter 6}

\section{Results and Discussions}

Finally, we look at the distribution of $\left(\cos \theta_{+}, \cos \theta_{-}\right)$and $\left(\cos \theta_{b}, \cos \theta_{\bar{b}}\right)$ in data for integrated luminosity of $5.1 \mathrm{fb}^{-1}$, which has been kept blinded until all studies on all possible systematic uncertainties are done.

Then we calculate unbinned likelihood by Equation 4.8 to obtain measured $\kappa\left(\kappa^{\text {meas }}\right)$.
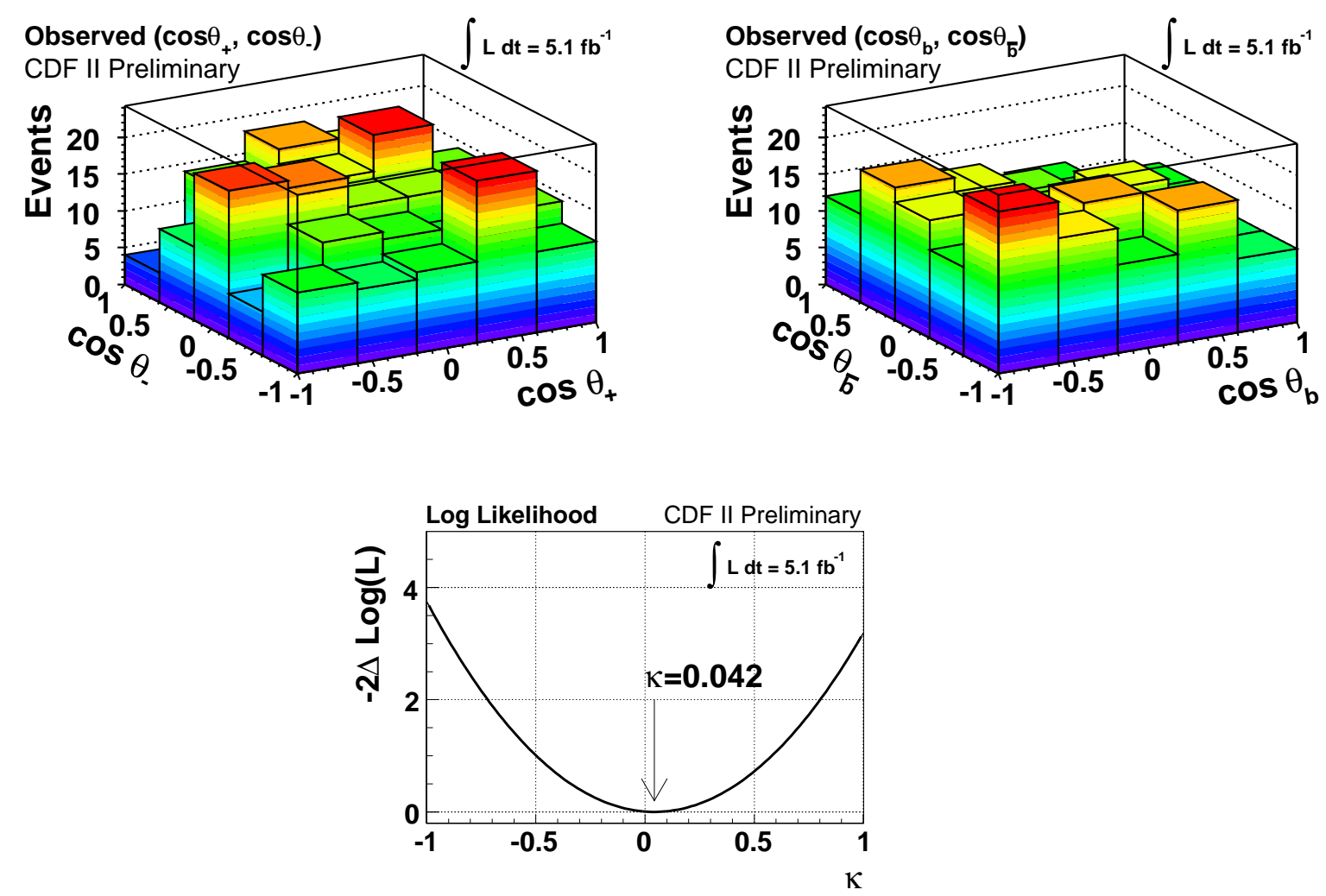

Figure 6.1: Upper left: the distribution of reconstructed $\left(\cos \theta_{+}, \cos \theta_{-}\right)$in 5 by 5 bins. Upper right: the distribution of reconstructed $\left(\cos \theta_{b}, \cos \theta_{\bar{b}}\right)$ in 5 by 5 bins Lower: $-2 \Delta \log \mathcal{L}$ as a function of assumed $\kappa$. The function is found to be minimum at $\kappa=0.042$. 
Figure 6.1 shows the distributions of reconstructed $\left(\cos \theta_{+}, \cos \theta_{-}\right)$and $\left(\cos \theta_{b}, \cos \theta_{\bar{b}}\right)$, and $-2 \Delta \log \mathcal{L}$ as a function of assumed $\kappa$.

From the function, we observe $\kappa^{\text {meas }}=0.042$.

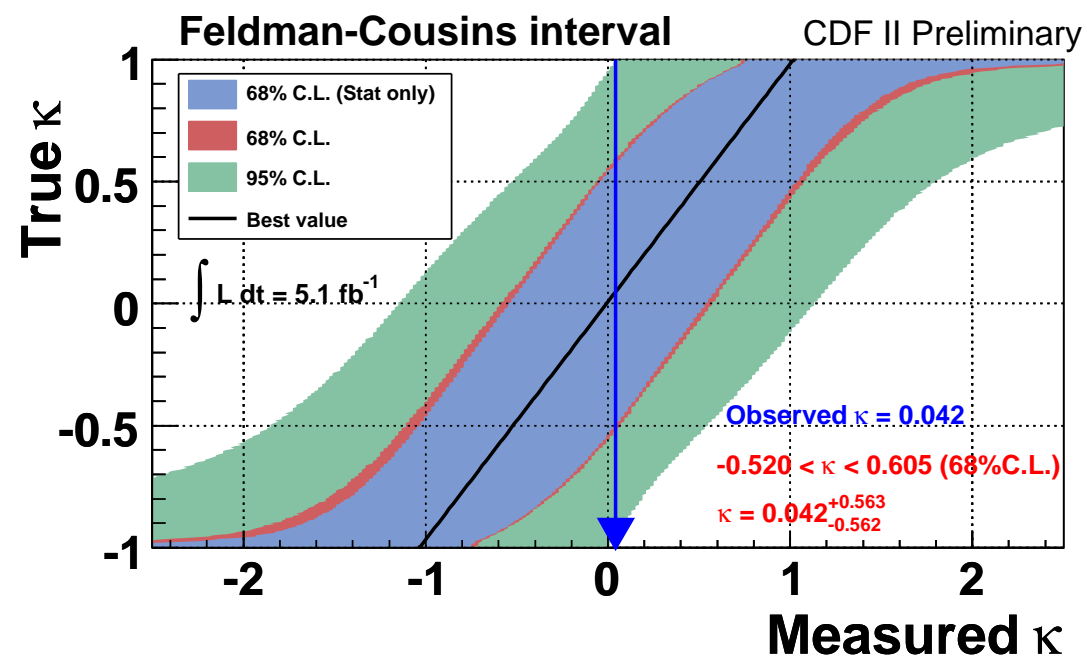

Figure 6.2: Feldman-Cousins confidence intervals with measured result. Interval at $68 \%$ C.L. on true $\kappa$ corresponding to $\kappa^{\text {meas }}=0.042$ is shown.

Figure 6.2 shows the Feldman-Cousins confidence intervals constructed in Section 4.6.4 and measured result $\kappa^{\text {meas }}=0.042$. From this we obtain the following results:

$$
-0.52<\kappa<+0.61(68 \% \text { C.L. })
$$

or

$$
\kappa=0.042_{-0.562}^{+0.563}
$$

on the assumption of $M_{\mathrm{top}}=172.5 \mathrm{GeV} / c^{2}$. In this analysis, we assume $M_{\text {top }}=172.5 \mathrm{GeV} / c^{2}$. The result will be changed if we assume another top mass. To look at top mass dependence of the result, we repeat the same analyses, but in which we change the assumption of top mass to each of $167.5,170.0,175.0$, and $177.5 \mathrm{GeV} / c^{2}$.

Figure 6.3 shows $68 \%$ C.L. intervals on $\kappa$ as a function of $M_{\text {top }}$. Solid line indicates center values of $\kappa$.

Due to signal sample statistics of each top mass, the center values are fluctuated. Since systematics except for signal sample statistics described in Section 5.1 are common among results for each top mass, The fluctuation is caused by only signal sample statistics. The numbers of dilepton candidates in $\mathrm{MC}$ with assumption that top mass $=167.5,170.0$ and $177.5 \mathrm{GeV} / c^{2}$ is $9 \mathrm{~K}$ each. Their signal sample statistics is about 0.08 . The numbers of dilepton candidates in $\mathrm{MC}$ with assumption that top mass $=175.0 \mathrm{GeV} / c^{2}$ is $30 \mathrm{~K}$. Its signal sample statistics is about 0.04. Considering this uncertainty, we do not see any conclusive top mass dependence of $\kappa$ measurement. 


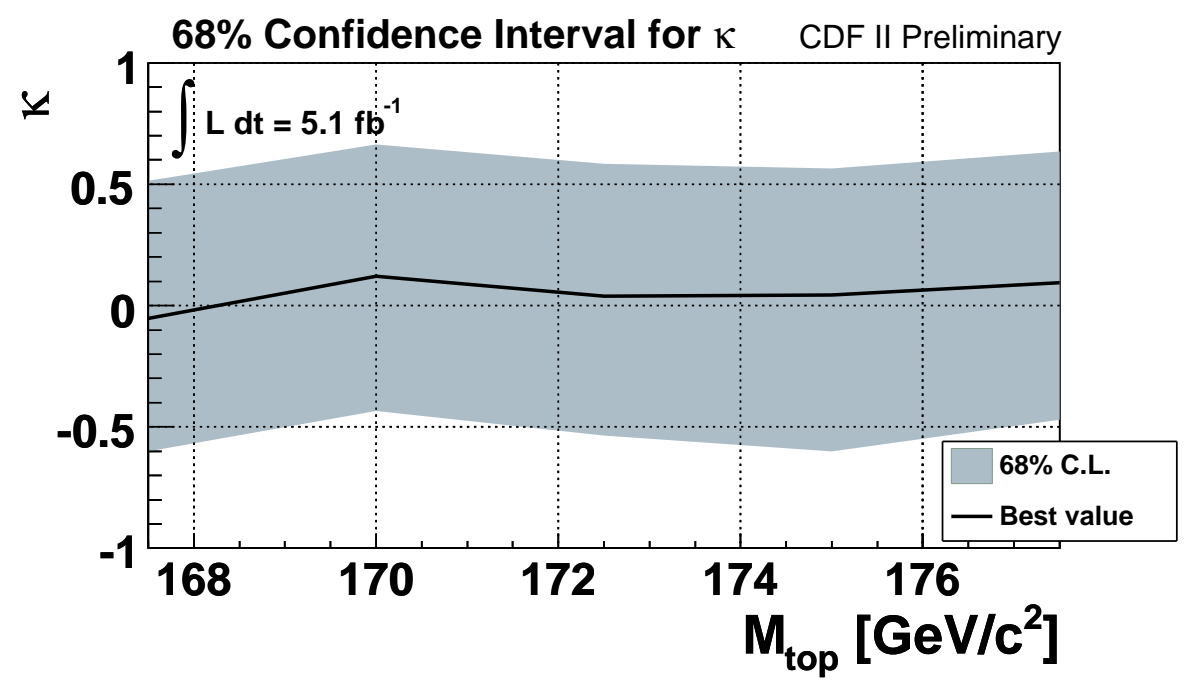

Figure 6.3: $68 \%$ C.L. intervals on $\kappa$ as a function of $M_{\text {top }}$. Solid line indicates center values of $\kappa$.

Comparing our result with previous measurements, our result of $\kappa=0.042_{-0.562}^{+0.563}$ is consistent with other measurements. Sensitivity of our measurement is comparable to the result reported by CDF II lepton plus jets channel of $0.72 \pm 0.64$ (stat) \pm 0.26 (syst), and little bit inferior to $\mathrm{D} \varnothing$ results of $0.66 \pm 0.23$ (stat + syst).

Currently, statistical uncertainty is dominating in our measurement. As data are accumulated, the sensitivity of the measurement is expected to increase. We will be able to analyses data of $10 \mathrm{fb}^{-1}$ in the near future. We consequently can reduce the uncertainty as integrated luminosity increases. 



\section{Chapter 7}

\section{Conclusion}

We have measured the spin correlation coefficient $\kappa$ of top and antitop quark in the beam basis at top quark pair production in proton-antiproton collisions at a center of mass energy of $1.96 \mathrm{TeV}$. Collected data corresponds to an integrated luminosity of $5.1 \mathrm{fb}^{-1}$ between March 2002 and June 2009 with the CDF Detector at Fermilab Tevatron.

We selected $t \bar{t}$ candidate events which contain two high transverse momentum charged leptons, muon or electron, high missing $E_{T}$ and two jets, originating from top quark pair production process. We found $334 t \bar{t}$ candidate events with an expected background of $86 \pm 14$ events.

We performed full kinematical reconstruction to obtain angular distribution of charged leptons and b-jets. Then we made the templates of $t \bar{t}$ signal as a function of $\kappa$ as well as background for each angular distribution using the simulation as well as data-based background modeling.

Using the templates, we fit the angular distribution of data, and we obtained measured $\kappa=0.042$ on the assumption of $M_{\text {top }}=172.5 \mathrm{GeV} / c^{2}$. And this corresponds to

$$
\begin{gathered}
-0.520<\kappa<+0.605(68 \% \text { C.L. }) \\
\text { or } \\
\kappa=0.042_{-0.562}^{+0.563} .
\end{gathered}
$$

Also, we can not find any conclusive top mass dependence on the $\kappa$ measurement.

This result indicates the null correlation rather than the existence of correlation. However, it does not mean the inconsistency with the Standard Model because this result has a large uncertainty. There is no hint for anomalous production or decay of top quark pairs.

This result is similar to one of $\mathrm{D} \emptyset$ result which obtained from differential angular distribution template in dilepton channel of $\kappa=0.10 \pm 0.45$ (stat+syst). Also, it indicates different tendency from other results (Lepton + jets channel at CDF, Lepton + jets channel at $\mathrm{D} \varnothing$, Dilepton channel at $\mathrm{D} \varnothing$ with matrix element method). These results indicate the existence of spin correlation in $t \bar{t}$ with 3 or more standard deviations.

Our analysis provides an independent measurement to others above. Besides, our result is currently limited by the statistical uncertainty. The total integrated luminosity of data collected with the CDF Detector at Fermilab Tevatron reached about $10 \mathrm{fb}^{-1}$. Since the statistical uncertainty is expected to be reduced by the factor $1 / \sqrt{2}$ as the statistics of 
the data sample increasing $N$ times, the sensitivity to the spin correlation coefficient will be improved by about $\sqrt{2}$ maximally. 


\section{Appendix A}

\section{Spin States at $t \bar{t}$ Pair Production}

\section{A.1 Production by $q \bar{q} \rightarrow t \bar{t}$}

In highly relativistic interactions, particle helicity is same as its chirality. For a fermion of energy $E \gg m$, we can use the following relations,

$$
\begin{aligned}
\frac{1}{2}\left(1-\gamma^{5}\right) u & =u_{L} \\
\frac{1}{2}\left(1+\gamma^{5}\right) u & =u_{R} .
\end{aligned}
$$

where $u$ indicates spinor of fermion, $u_{L}$ and $u_{R}$ indicate spinor of right-handed fermion and spinor of left-handed fermion, respectively, and $\frac{1}{2}\left(1 \pm \gamma^{5}\right)$ project out the helicity $\lambda= \pm \frac{1}{2}$ components of a spinor, respectively.

Also, for antifermion we can use the following relations,

$$
\begin{aligned}
& \frac{1}{2}\left(1-\gamma^{5}\right) v=v_{R} \\
& \frac{1}{2}\left(1+\gamma^{5}\right) v=v_{L} .
\end{aligned}
$$

where $v$ indicates spinor of antifermion, $v_{R}$ and $v_{L}$ indicate spinor of right-handed antifermion and spinor of left-handed antifermion, respectively, and $\frac{1}{2}\left(1 \pm \gamma^{5}\right)$ project out the helicity $\lambda=\mp \frac{1}{2}$ components of a spinor, respectively.

Strong interaction is mediated by gluon and it has vector structure. Therefore, quark antiquark annihilation is described as the following equation.

$$
\bar{v}^{\prime} \gamma^{\mu} u=\left(\bar{v}_{L}^{\prime}+\bar{v}_{R}\right) \gamma^{\mu}\left(u_{L}+u_{R}\right)=\bar{v}_{R} \gamma^{\mu} u_{L}+\bar{v}_{L}{ }_{L} \gamma^{\mu} u_{R}
$$

The proof is as follows.

$$
\begin{aligned}
& \bar{v}_{L}=v_{L}^{\dagger} \gamma^{0}=v^{\dagger} \frac{1}{2}\left(1+\gamma^{5}\right) \gamma^{0}=\bar{v} \frac{1}{2}\left(1-\gamma^{5}\right) \\
& \bar{v}_{R}=v_{R}^{\dagger} \gamma^{0}=v^{\dagger} \frac{1}{2}\left(1-\gamma^{5}\right) \gamma^{0}=\bar{v} \frac{1}{2}\left(1+\gamma^{5}\right)
\end{aligned}
$$




$$
\begin{aligned}
& \text { since } \gamma^{5}=\gamma^{5 \dagger} \text { and } \gamma^{5} \gamma^{0}=-\gamma^{0} \gamma^{5} \text {. Hence, } \\
& \qquad \begin{aligned}
\bar{v}_{L}^{\prime} \gamma^{\mu} u_{L} & =\frac{1}{4} \bar{v}^{\prime}\left(1-\gamma^{5}\right) \gamma^{\mu}\left(1-\gamma^{5}\right) u=\frac{1}{4} \bar{v}^{\prime} \gamma^{\mu}\left(1+\gamma^{5}\right)\left(1-\gamma^{5}\right) u=0 \text { (A.8) } \\
\bar{v}_{R}{ }^{\mu} \gamma^{\mu} u_{R} & =\frac{1}{4} \bar{v}^{\prime}\left(1+\gamma^{5}\right) \gamma^{\mu}\left(1+\gamma^{5}\right) u=\frac{1}{4}{\overline{v^{\prime}}}^{\mu}\left(1-\gamma^{5}\right)\left(1+\gamma^{5}\right) u=0, \text { (A.9) }
\end{aligned}
\end{aligned}
$$

where we have used $\gamma^{5} \gamma^{\mu}=-\gamma^{\mu} \gamma^{5}$ and $\left(\gamma^{5}\right)^{2}=1$.

Equation A.5 shows that initial quark and antiquark can only have opposite helicity states. Therefore, quark and antiquark annihilation process is in the following total angular momentum state,

$$
J=1, J_{z}= \pm 1
$$

Top quark spin and antitop quark spin are $\frac{1}{2}$ each and they move oppositely in the $t \bar{t}$ rest frame, therefore top quark and antitop quark will have opposite helicity if they have the same spin directions.

$q \bar{q}$ annihilation process at Tevatron is high energy interaction but their velocity is smaller than velocity of light. So, small amount of $J=1, J_{z}=0$ state exists. $q \bar{q} \rightarrow t \bar{t}$ process is in following total angular momentum states,

$$
\begin{array}{llrl}
J=1, J_{z}=+1 & : & |+>|+> \\
J=1, J_{z}=0 & : & \frac{1}{\sqrt{2}}[|+>|->+|->|+>] \\
J=1, J_{z}=-1 & : & & |->|->
\end{array}
$$

where $\mid+>$ and $\mid->$ indicate "spin up" state and "spin down" state, respectively.

The exact energy dependence of the spin states for beamline basis can be determined by looking at the matrix elements for the production of like spin and unlike spin top quark pairs via $q \bar{q} \rightarrow t \bar{t}$. These matrix elements are given in Equations A.10 and A.11 [66].

$$
\begin{aligned}
& \sum_{\uparrow \uparrow, \downarrow \downarrow}|\mathcal{M}(q \bar{q} \rightarrow t \bar{t})|^{2}=8 g^{4} \frac{\beta^{2}\left(1-\beta^{2}\right) \sin ^{2} \theta^{*}}{\left(1-\beta \cos \theta^{*}\right)^{2}} \\
& \sum_{\uparrow \downarrow, \downarrow \uparrow}|\mathcal{M}(q \bar{q} \rightarrow t \bar{t})|^{2}=8 g^{4}\left[1+\frac{\left(1-\beta \cos \theta^{*}-\beta^{2} \sin ^{2} \theta^{*}\right)^{2}}{\left(1-\beta \cos \theta^{*}\right)^{2}}\right]
\end{aligned}
$$

In these equations, $g$ is the QCD coupling strength, $\theta^{*}$ is the top quark scattering angle with respect to the proton beam in the $t \bar{t}$ center of mass system, and $\beta=v / c$ is top quark velocity, which depends on the $t \bar{t}$ pair production energy and is limited to be between 0 and 1.

Notice the factor $\beta^{2}\left(1-\beta^{2}\right)$ in the like-spin pair amplitude (Equation A.10). It supplies suppression of this component for both small and large value of $\beta$. In contrast, the unlikespin pair amplitude (Equation A.11) contains a contribution which is independent of $\beta$. 


\section{A.2 Production by $g g \rightarrow t \bar{t}$}

Because gluon is a massless particle, gluon spin state is in $J=1, J_{z}= \pm 1$ state. Therefore, total angular momentum projection of gluon pair can be in $J_{z}=+2,0$, or -2 . Also, total angular momentum of gluon pair can be in $J=2,1,0$ state.

When $t \bar{t}$ pairs are produced near kinematic threshold, pair of top and antitop quark do not have orbital angular momentum. Since $t \bar{t}$ pair can not be in $J_{z}= \pm 2$ state, $g g \rightarrow t \bar{t}$ process can not be in $J_{z}=2$ state.

Therefore, $g g \rightarrow t \bar{t}$ process can be in $J=1, J_{z}=0$ or $J=0, J_{z}=0$ state.

$$
\begin{aligned}
& J=1, J_{z}=0: \quad \sqrt{\frac{1}{2}}\left|+1,-1>-\sqrt{\frac{1}{2}}\right|-1,+1> \\
& J=0, J_{z}=0: \quad \sqrt{\frac{1}{3}}\left|+1,-1>-\sqrt{\frac{1}{3}}\right| 0,0>+\sqrt{\frac{1}{3}} \mid-1,+1>
\end{aligned}
$$

where, $\mid J_{z}^{1}, J_{z}^{2}>$ indicate $J_{z}$ of each gluon. Each gluon can not be in $J_{z}=0$ (longitudinal) state. Therefore $J=0, J_{z}=0$ state is rewritten as follows.

$$
J=0, J_{z}=0: \sqrt{\frac{1}{2}}[|+1,-1>+|-1,+1>]
$$

In addition, because gluon is a boson, when one swaps two gluons, the wavefunction of the system has to be unchanged. Therefore, $J=1, J_{z}=0$ state is excluded and $J=0, J_{z}=0$ state is only allowed, if the wavefunction of color part in two-gluon system is a symmetric with exchange of two gluons.

The exact energy dependence can be seen by looking directly at the matrix elements for $g g \rightarrow t \bar{t}$ production, as given in Equations A.15 and A.16 with the angular factor $\mathcal{Y}$ defined in Equation A.17 [66].

$$
\begin{aligned}
\sum_{\uparrow \uparrow, \downarrow \downarrow}|\mathcal{M}(g g \rightarrow t \bar{t})|^{2} & =\frac{16}{3} g^{4} \mathcal{Y}\left(1-\beta^{2}\right) \\
& \times\left[1+\beta^{2}+\beta^{2} \cos ^{2} \theta^{*}+2 \beta^{3} \sin ^{2} \theta^{*} \frac{\beta-\cos \theta^{*}}{(1-\beta \cos \theta)^{2}}\right] \\
\sum_{\uparrow \downarrow, \downarrow \uparrow}|\mathcal{M}(g g \rightarrow t \bar{t})|^{2} & =\frac{16}{3} g^{4} \mathcal{Y} \beta^{2} \sin ^{2} \theta^{*} \\
& \times\left[1+\frac{\left(1-\beta^{2}\right)^{2}+\left(1-\beta \cos \theta^{*}-\beta^{2} \sin ^{2} \theta^{*}\right)^{2}}{\left(1-\beta \cos \theta^{*}\right)^{2}}\right] \\
\mathcal{Y} & =\frac{7+9 \beta^{2} \cos ^{2} \theta^{*}}{\left(1-\beta^{2} \cos ^{2} \theta^{*}\right)^{2}}
\end{aligned}
$$

Equation A.15 shows that the like-spin pairs coming from gluon-gluon fusion will be suppressed for large $\beta$, while Equation A.16 tells us that unlike-spin pairs are disfavored at low $\beta$.

The breakdown of the total $t \bar{t}$ cross section into like- and unlike-spin pairs as a function of the $t \bar{t}$ invariant mass is given in Figure A.1 for the Tevatron using the beamline basis [66]. 


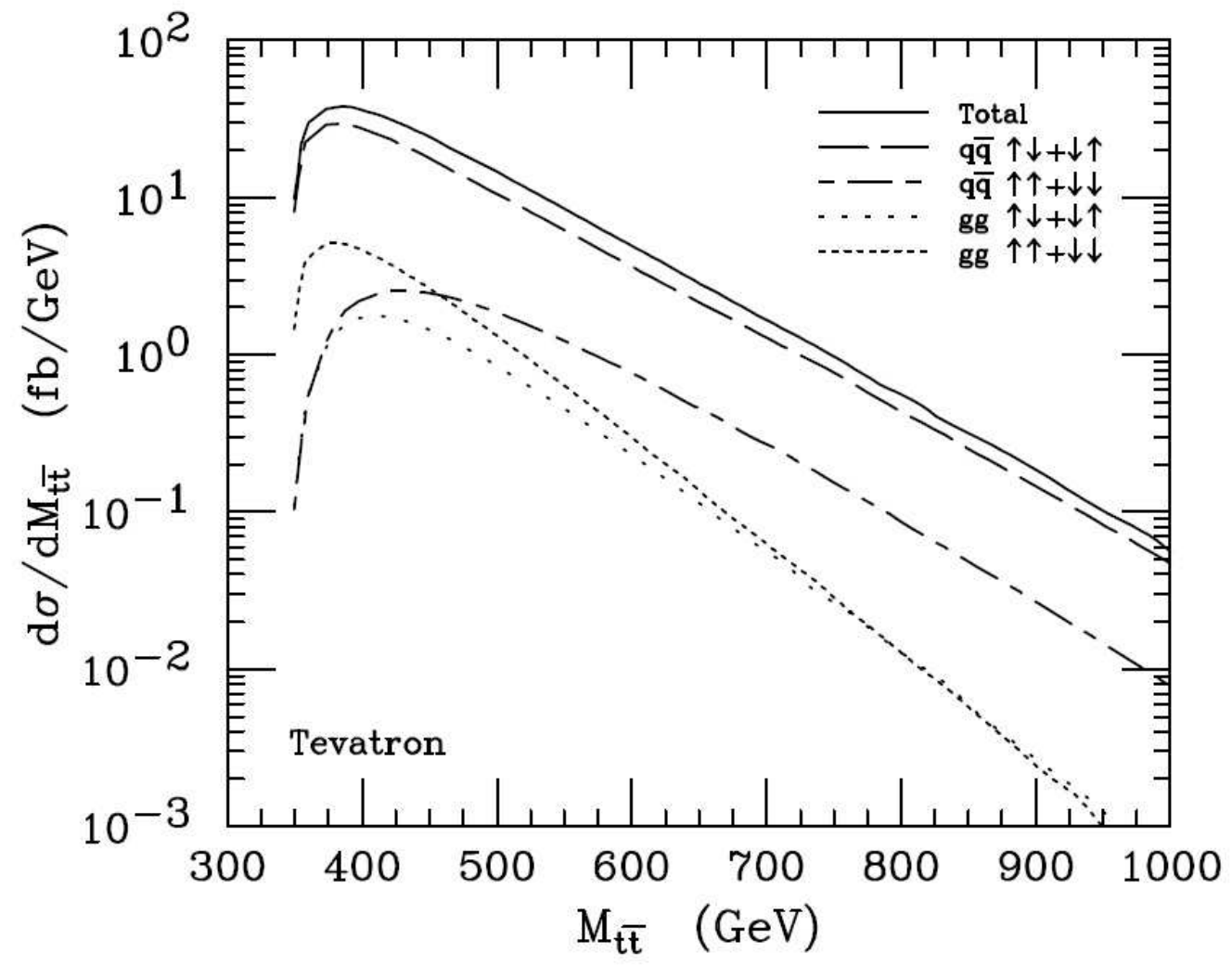

Figure A.1: Differential cross section for $t \bar{t}$ production as a function of the $t \bar{t}$ invariant mass, $M_{t \bar{t}}$, for the Tevatron with center of mass energy $2.0 \mathrm{TeV}$, decomposed into $\uparrow \downarrow+\downarrow \uparrow$ and $\uparrow \uparrow+\downarrow \downarrow$ spins of the $t \bar{t}$ pair using beamline basis for both $q \bar{q}$ and $g g$ components. [66] 


\section{Appendix B}

\section{P, CP of $t \bar{t}$ System}

Figure B.1 illustrates spin configurations of $t \bar{t}$ system and their $P$ and $C P$ reversal states.

Since helicities are flipped under $P$ reversal,

$$
\begin{aligned}
& P\left(N_{\uparrow \downarrow}\right)=N_{\downarrow \uparrow}, \\
& P\left(N_{\downarrow \downarrow}\right)=N_{\uparrow \uparrow},
\end{aligned}
$$

where $N_{s_{t} \bar{s}_{t}}$ indicates number of $t, \bar{t}$ in $s_{t}, \bar{s}_{t}$ state with respect to the quantization axis for the top quark.

Since top and antitop are exchanged under $C$ reversal,

$$
C P\left(N_{\downarrow \downarrow}\right)=C\left(N_{\uparrow \uparrow}\right)=N_{\uparrow \uparrow} .
$$

Therefore, if $P$ is conserved at $t \bar{t}$ production, $N_{\uparrow \downarrow}=N_{\downarrow \uparrow}$, or, vise versa, if $P$ is violated, there would be an asymmetry between $N_{\uparrow \downarrow}$ and $N_{\downarrow \uparrow}$. If $C P$ is conserved at $t \bar{t}$ production, $N_{\downarrow \downarrow}=N_{\uparrow \uparrow}$, or if $C P$ is violated, there would be an asymmetry between $N_{\downarrow \downarrow}$ and $N_{\uparrow \uparrow}$.
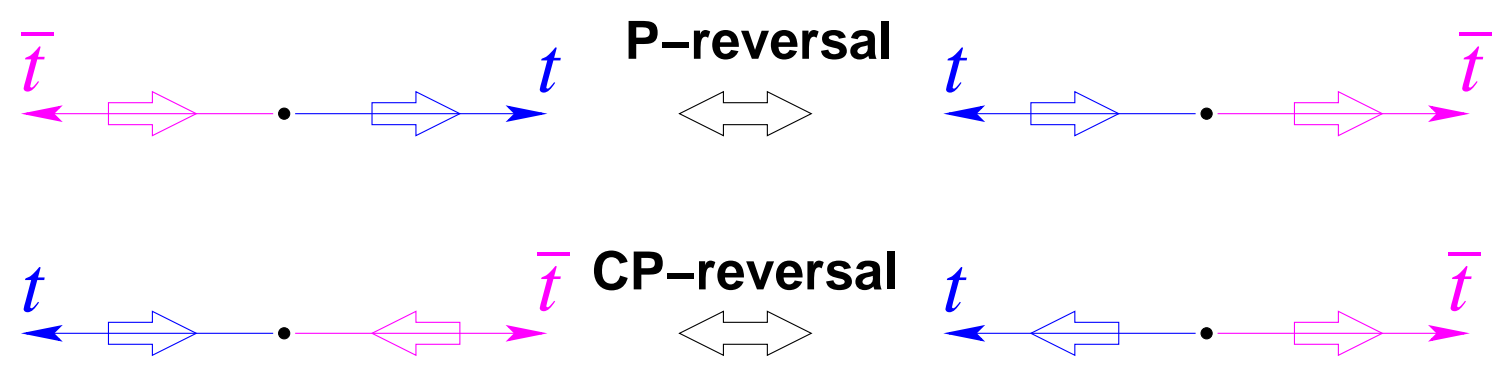

Figure B.1: Spin configurations of $t \bar{t}$ system and their $\mathrm{P}$ and $\mathrm{CP}$ reversal states. In the case above, $P\left(N_{\uparrow \downarrow}\right)=N_{\downarrow \uparrow}, P\left(N_{\downarrow \downarrow}\right)=N_{\uparrow \uparrow}$ and $C P\left(N_{\downarrow \downarrow}\right)=C\left(N_{\uparrow \uparrow}\right)=N_{\uparrow \uparrow}$. So if $P$ and $C P$ are conserved, $N_{\uparrow \downarrow}=N_{\downarrow \uparrow}$ and $N_{\downarrow \downarrow}=N_{\uparrow \uparrow}$.

Next, we consider the effect of $P$ reversal and $C P$ reversal on $\cos \theta(\theta$ denotes the angle between the quantization axis and the flight direction of the decay particles from the top quark in the top quark rest frame). Under $P$ reversal $\cos \theta_{ \pm}$changes to $-\cos \theta_{ \pm}$ since helicity states of top quark and antitop quark is flipped under $P$ reversal. Under $C$ 
reversal $\cos \theta_{ \pm}$changes to $-\cos \theta_{\mp}$ since top and antitop are exchanged under $C$ reversal. Therefore, Under $C P$ reversal, $\cos \theta_{ \pm}$changes to $\cos \theta_{\mp}$. 


\section{Bibliography}

[1] F. Abe et al., Observation of top quark production in $p \bar{p}$ collisions with the Collider Detector at Fermilab, Phys. Rev. Lett. 74, 2626 (1995).

[2] S. Abachi et al., Observation of top quark, Phys. Rev. Lett. 74, 2632 (1995).

[3] DONUT collaboration, K. Kodama et al., Observation of tau neutrino interactions, Phys. Lett. B504, 218 (2001).

[4] D. J. Gross and F. Wilczek, Asymptotically free gauge theories, Phys. Rev. D8, 3633 (1973).

[5] H. D. Politzer, Asymptotic freedom: An approach to strong interactions, Phys. Rept. 14, 129 (1974).

[6] S. L. Glashow, Partial symmetries of weak interactions, Nucl. Phys. 22, 579 (1961).

[7] A. Salam and J. C. Ward, Electromagnetic and weak interactions, Phys. Lett. 13, 168 (1964).

[8] S. Weinberg, A model of leptons, Phys. Rev. Lett. 19, 1264 (1967).

[9] N. Cabibbo, Unitary Symmetry and Leptonic Decays, Phys. Rev. Lett. 10, 531 (1963).

[10] M. Kobayashi and T. Masukawa, CP Violation in the Renormalizable Theory of Weak Interaction, Prog. Theor. Phys. 49, 652 (1973).

[11] P. W. Higgs, Broken symmetries and the masses of gauge bosons, Phys. Rev. Lett. 13, 508 (1964).

[12] LEP working Group for Higgs boson searches and ALEPH collaboration and DELPHI collaboration and L3 collaboration and OPAL collaboration, R. Barate and others., Search for the standard model Higgs boson at LEP, Phys. Lett. B565, 61 (2003).

[13] Tevatron New Phenomina and Higgs Working Group, CDF collaboration, D $\varnothing$ collaboration, Combined CDF and D0 Upper Limits on Standard Model Higgs Boson Production with up to $8.6 \mathrm{fb}^{-1}$ of Data, (2011), arXiv:hep-ex/1107.5518.

[14] The LEP Electroweak Working Group, http://lepewwg.web.cern.ch/LEPEWWG/. 
[15] W. Bernreuther et al., Top quark pair production and decay at hadron colliders, Nucl. Phys. B690, 81 (2004).

[16] E. Rice et al., Search for structure in $\sigma\left(\mathrm{e}^{+} \mathrm{e}^{-} \rightarrow\right.$ hadrons $)$ between $\sqrt{s}=10.34$ and 11.6 GeV, Phys. Rev. Lett. 48, 906 (1982).

[17] CDF collaboration, D0 collaboration, Tevatron Electroweak Working Group, E. Rice et al., Combination of CDF and D0 Results on the Top-Quark Mass, arXiv:hepex/0404010.

[18] M. Cacciari et al., The $t \bar{t}$ cross-section at 1.8 and $1.96 \mathrm{TeV}$ : A study of the systematics due to parton densities and scale dependence, J. High Energy Phys. 0404, 068 (2004).

[19] A. F. Falk and M. E. Peskin, Production, decay, and polarization of excited heavy hadrons, Phys. Rev. D49, 3320 (1994).

[20] M. Beneke et al., Top quark physics, CERN-TH/2000-100 (2000), arXiv:hep$\mathrm{ph} / 0003033$.

[21] G. Mahlon and S. J. Parke, Maximizing spin correlations in top quark pair production at the Tevatron, Phys. Lett. B411, 173 (1997).

[22] I. Corcella, G. Knowles et al., HERWIG 6: An Event generator for hadron emission reactions with interfering gluons (including supersymmetric processes), J. High Energy Phys. 0101, 010 (2001).

[23] CTEQ collaboration, J. Lai, H. L. Huston et al., Global QCD analysis of parton structure of the nucleon: CTEQ5 parton distributions, Eur. Phys. J. C12, 375 (2000),

[24] N. Arai, M. Okada and K. Smolek, Top Quark Spin Correlations in the RandallSundrum Model at the CERN Large Hadron Collider, Phys. Rev. D75, 095008 (2007).

[25] CDF Collaboration, T. Schwarz et al., Combination of the Forward-Backward Asymmetry in the Top Pair Production from L+J and DIL Channels using $5 \mathrm{fb}^{-1}$, CDF Conference Note 10584 (2011).

[26] D. W. Jung et al., Model Independent analysis of the forward-backward asymmetry of top quark production at the Tevatron, Phys. Lett. B691, 238 (2010).

[27] $\mathrm{D} \varnothing$ collaboration, V. Abazov et al., Measurement of spin correlation in $t \bar{t}$ production using a matrix element approach, Phys. Rev. Lett. 107, 032001 (2011).

[28] D $\varnothing$ collaboration, V. Abazov et al., Measurement of spin correlation in $t \bar{t}$ production using dilepton final sates, Phys. Lett. B702, 16 (2011).

[29] T. A. collaboration, Measurement of spin correlation in $t \bar{t}$ production from $p p$ collisions at $\sqrt{s}=7 \mathrm{TeV}$ using the ATLAS detector, ATLAS-CONF-2011-117 (2011). 
[30] CDF collaboration, T. Aaltonen et al., Measurement of $t \bar{t}$ Spin Correlation in $p \bar{p}$ Collisions Using the CDF II Detector at the Tevatron, Phys. Rev. D83, 031104 (2011).

[31] D $\varnothing$ collaboration, V. Abazov et al., Evidence for spin correlation in $t \bar{t}$ production, Phys. Rev. Lett. 108, 032004 (2011).

[32] E. W. J. Cockroft, Experiments with High Velocity Positive Ions. II. The Disintegration of Elements by High Velocity Protons., Proc. of the Royal Soc. of London A137, 229 (1932).

[33] J. Marriner, Stochastic cooling overview, Nucl. Instrum. and Meth. A532, 11 (2004),

[34] S. Nagaitsev et al., Experimental demonstration of relativistic electron cooling, Phys. Rev. Lett. 96, 044801 (2006).

[35] On behalf of the CDF, C. S. Hill, Operational experience and performance of the CDFII silicon detector, Nucl. Instrum. and Meth. A530, 1 (2004).

[36] CDF collaboration, A. Sill, CDF Run II silicon tracking projects, Nucl. Instrum. and Meth. A447, 1 (2000).

[37] CDF collaboration, A. A. Affolder et al., Intermediate silicon layers detector for the CDF experiment, Nucl. Instrum. and Meth. A453, 84 (2000).

[38] CDF collaboration, A. A. Affolder et al., CDF central outer tracker, Nucl. Instrum. and Meth. A526, 249 (2004).

[39] CDF collaboration, D. Acosta et al., A time-of-flight detector in CDF-II, Nucl. Instrum. and Meth. A518, 605 (2004).

[40] CDF collaboration, L. Balka et al., The CDF Central Electromagnetic Calorimeter, Nucl. Instrum. and Meth. A267, 272 (1988).

[41] CDF collaboration, M. G. Albrow et al., The CDF plug upgrade electromagnetic calorimeter: Test beam results, Nucl. Instrum. and Meth. A480, 524 (2002).

[42] G. Apollinari, K. Goulianos, P. Melese, and M. Lindgren, Shower maximum detector for the CDF plug upgrade calorimeter, Nucl. Instrum. and Meth. A412, 515 (1998).

[43] CDF collaboration, S. Bertolucci et al., The CDF Central and Endwall Hadron Calorimeter, Nucl. Instrum. and Meth. A267, 301 (1988).

[44] G. Ascoli et al., CDF Central Muon Detector, Nucl. Instrum. and Meth. A268, 33 (1988).

[45] D. Acosta et al., The performance of the CDF luminosity monitor, Nucl. Instrum. and Meth. A494, 57 (2002). 
[46] E. J. Thomson et al., Online track processor for the CDF upgrade, IEEE Trans. Nucl. Sci. 49, 1063 (2002).

[47] CDF collaboration, B. Ashmanskas et al., The CDF silicon vertex trigger, Nucl. Instrum. and Meth. A518, 532 (2004),

[48] A. Bhatti et al., Determination of the jet energy scale at the collider detector at Fermilab, Nucl. Instrum. and Meth. A566, 375 (2006),

[49] M. L. Mangano, M. Moretti, F. Piccinini, R. Pittau, and A. D. Polosa, ALPGEN, a generator for hard multiparton processes in hadronic collisions, J. High Energy Phys. 07, 001 (2003),

[50] M. L. Mangano et al., Multijet matrix elements and shower evolution in hadronic collisions: $W b \bar{b}+n$ jets as a case study, Nucl. Phys. B632, 343 (2002).

[51] F. Caravaglios et al., A New approach to multijet calculations in hadron collisions, Nucl. Phys. B539, 215 (1999).

[52] T. Sjostrand et al., , Comp. Phys. Commun. 135, 238 (2001).

[53] J. Alwall et al., MdGraph/MadEvent v4: The New Web Generation, J. High Energy Phys. 0709, 028 (2007).

[54] CTEQ collaboration, P. M. Nadolsky et al., Implication of CTEQ gloval analysis for collider observables, Phys. Rev. D78, 013004 (2008).

[55] GEANT4 collaboration, S. Agostinelli et al., GEANT4: A Simulation toolkit, Nucl. Instrum. and Meth. A506, 250 (2003).

[56] E. Gerchtein and M. Paulini, CDF detector simulation framework and performance, (2003), arXiv:physics/0306031.

[57] CDF collaboration, T. Aaltonen et al., Top Dilepton Cross Section in $5.1 \mathrm{fb}^{-1}$ using the DIL Selection, CDF Public Note 10163 (2010).

[58] R. Chakravarti, I.M. Laha and J. Roy, Kolmogorov-Smirnov (K-S) test, Handbook of methods of applied Statistics Volume I, 392 (1967).

[59] G. Feldman and R. Cousins, A Unified approach to the classical statistical analysis of small signals, Phys. Rev. D57, 3873 (1998).

[60] J. Gentle, Cholesky Factrization, \$3.2.2. in Numerical Linear Algebra for Application in Statistics. Berline: Springer-Verlag, 93 (1998).

[61] N. Kidonakis and R. Vogt, Next-to-next-to-leading order soft-gluon corrections in top quark hadroproduction, Phys. Rev. D68, 114014 (2003).

[62] S. Frixione and B. Webber, Matching NLO QCD computations and parton shower simulations, J. High Energy Phys. 0206, 029 (2002). 
[63] S. Frixione et al., Matching NLO QCD and parton showers in heavy flavor production, J. High Energy Phys. 0308, 007 (2003).

[64] D. Wicke and P. Skands, Non-perturbative QCD effects and the top mass at the Tevatron, Eur. Phys. J. C52, 133 (2007).

[65] P. Skands, The Perugia Tunes, arXiv:hep-ph/0905.3418.

[66] G. Mahlon and S. J. Parke, Angular Correlations in Top Quark Pair Production and Decay at Hadron Colliders, Phys. Rev. D53, 4886 (1996). 